University of Tennessee Health Science Center UTHSC Digital Commons

\title{
Molecular Mechanisms of Metabolic Reprogramming in Pancreatic Cancer
}

\author{
Sonam Kumari \\ University of Tennessee Health Science Center
}

Follow this and additional works at: https://dc.uthsc.edu/dissertations

Part of the Oncology Commons

\section{Recommended Citation \\ Kumari, Sonam (0000-0002-6052-9972), "Molecular Mechanisms of Metabolic Reprogramming in Pancreatic Cancer" (2019). Theses and Dissertations (ETD). Paper 490. http://dx.doi.org/10.21007/ etd.cghs.2019.0482.}

This Dissertation is brought to you for free and open access by the College of Graduate Health Sciences at UTHSC Digital Commons. It has been accepted for inclusion in Theses and Dissertations (ETD) by an authorized administrator of UTHSC Digital Commons. For more information, please contact jwelch30@uthsc.edu. 


\title{
Molecular Mechanisms of Metabolic Reprogramming in Pancreatic Cancer
}

\begin{abstract}
Pancreatic cancer ranks as third deadliest cancer worldwide in both men and women. The lack of diagnostic methods, chemo-resistance and invasiveness of the disease diminishes the overall survival rate in patients. Dysregulated glucose metabolism and several molecular determinants including oncogenic signaling pathways play pivotal roles in the development, progression and metastasis of pancreatic cancer. The aberrant glucose metabolism is the most prevalent key factor that influences pancreatic tumorigenesis. The cancer cells of pancreas utilize oxygen for the breakdown of glucose to lactate, which is known as Warburg effect. This phenotype of pancreatic cancer encourages proliferation, growth, migration and invasive phenotypes of cancer cells that worsens the disease. The upstream regulatory molecules responsible for deviant activity of glucose transporters during aberrant glucose metabolism was not very well defined. Thus, identification of new molecular determinants that regulate glucose metabolic pathway would provide promising therapeutic outcomes. Our lab has established the role of MUC13 protein in pancreatic cancer development. Another interesting molecule, Protein Kinase D1 has been reported to play essential role in hypoxic metabolism in squamous cell carcinoma, and glucose uptake in cardiac hypertrophy. Also, it has been extensively studied in our research group, and has been suggested by some other groups to have an essential role in pancreatic cancer. However, its role in regulating glucose metabolism in pancreatic cancer remains elusive. In this dissertation, we investigated that MUC13 and PKD1 proteins are involved in abrupt glucose metabolism in pancreatic cancer cells. We have demonstrated that targeted overexpression of these oncogenes upregulates key oncogenic signaling components involved in aberrant glucose metabolism in pancreatic cancer cells. We also investigated a natural agent Steviol that restored glucose metabolism in pancreatic cancer cells via repression of MUC13 and PKD1 expression. MUC13 is a transmembrane glycoprotein which has an elevated expression in pancreatic cancer. Due to the presence of a cytoplasmic domain, it confers kinase activity and regulates many signaling processes. Therefore, we investigated the effect of MUC13 in metabolic reconditioning of pancreatic cancer. As a result, it was observed that presence of MUC13 in pancreatic cancer upregulated glucose uptake and lactate secretion. This in turn led to more aggressive behavior of the tumor, as evidenced by enhanced proliferation, migration and invasive characteristics of the cells as compared to MUC13 null cells. Interestingly, we observed the interaction of MUC13 with Glut-1 protein, which influences glucose uptake in cancer cells. This was confirmed through various assays such as: immunoprecipitation, immunofluorescence, co-capping and proximity ligation assay. The interpretation of inhibitor results envisaged the role of NFKB pathway during the molecular interactions of MUC13 and Glut-1. To understand the clinical importance of this mechanism, we evaluated this interaction in tumor samples from the patients with pancreatic disease in advanced stages. Our findings fortified the mechanistic role of MUC13 in rewiring of aberrant glucose metabolism. Altogether, we believed that MUC13 has clinical implication as a key molecule that might be responsible for dysregulated glucose metabolism. As discussed earlier about the paucity of associated proteins related to dysregulated glucose metabolism, we further investigated the possible key players with kinase domain for association and activation with other signaling molecules. Protein Kinase D1 (PKD1), a Ser/Thr kinase has been shown to be involved in progression of pancreatic cancer. In this study, we established PKD1 as a novel molecular target and its involvement in regulating aberrant glucose metabolism in pancreatic cancer. We observed that PKD1 was overexpressed in pancreatic cancer tissues compared to normal human tissues. We also found constitutive expression of PKD1 in various pancreatic cancer cells as compared to normal pancreatic ductal epithelial cells. The overexpression of PKD1 in low PKD1 expressing pancreatic cancer cells enhanced tumorigenic characteristics. We observed that specific knockdown of PKD1 inhibited key oncogenic signaling components in pancreatic cancer cells suggesting its oncogenic role in pancreatic cancer. Additionally, during the PKD1 overexpression, cells displayed increased glucose consumption and lactate production. This suggested role of PKD1 in metabolic
\end{abstract}


reprogramming in pancreatic cancer. Also, we observed that PKD1 stimulates the glucose uptake through mTORC1, a component of mTOR signaling pathway. Additionally, the knockdown of PKD1 promoted the chemosensitivity of cells towards gemcitabine and 2DG (2-deoxyglucose). Overall, these results indicated a significant function of PKD1 in rewiring of glucose metabolism in pancreatic cancer. It was mandating to target and counteract the aberrant glucose metabolism along with the proteins identified in our previous results. In this perspective herein, we proposed steviol, a natural sweetener from plant origin to assess its inhibitory action towards dysregulated glucose metabolism and its associated proteins (MUC13 and PKD1). The reason behind steviol selection for this approach includes that it mimics the glucose molecule that facilitates enhanced uptake of drug (steviol) within the cells and on the other side, it provides glucose homeostasis to adjacent normal cells. These features made steviol as dual functional drug by overtaking other natural drugs for promising results. The exposure of steviol inhibited the proliferative (IC50: 10mM) and clonogenic abilities of pancreatic cancer cells. It also suppressed the cell migration and cell invasion capabilities of pancreatic cancer cells. Further, steviol caused the selective inhibition of intracellular glucose intake and lactate accumulation in a concentration dependent manner. The selective inhibition of MUC13 and PKD1 (upstream key proteins of glucose metabolism) that caused the disruption of glucose intake in cancer cells, was observed during steviol treatment. Secondly, steviol interfered with translation-initiation machinery, causing destabilization of the cellular functionality. The impairment of cellular translation process had promoted G1 cell cycle arrest in cells due to the lack of G1-S transition proteins during drug exposure. Collectively, all these events engendered the cellular integrity and directed towards activation of apoptosis. In conclusion, we evaluated the role of MUC13 and PKD1 in aberrant glucose metabolism leading to growth and aggressive metastatic phenotypes of pancreatic cancer. We also demonstrated efficacy of steviol to repress aberrant glucose metabolism via suppression of MUC13 and PKD1 proteins.

\section{Document Type}

Dissertation

Degree Name

Doctor of Philosophy (PhD)

\section{Program}

Pharmaceutical Sciences

Research Advisor

Meena Jaggi, Ph.D.

\section{Keywords}

Cancer Biology, Glucose Metabolism, Pancreatic Cancer

\section{Subject Categories}

Medicine and Health Sciences | Oncology 
Molecular Mechanisms of Metabolic Reprogramming in Pancreatic Cancer

\author{
A Dissertation \\ Presented for \\ The Graduate Studies Council \\ The University of Tennessee \\ Health Science Center
}

In Partial Fulfillment

Of the Requirements for the Degree

Doctor of Philosophy

From The University of Tennessee

By

Sonam Kumari

May 2019 
Copyright (C) 2019 by Sonam Kumari.

All rights reserved. 


\section{DEDICATION}

I want to dedicate my dissertation to my beloved parents,

Mr. Nalni Ranjan Saha and Mrs. Vidya Saha, whose encouragement enabled me to achieve this success. 


\section{ACKNOWLEDGEMENTS}

I want to sincerely acknowledge my advisor Dr. Meena Jaggi for her constant guidance throughout the Ph.D program. I also want to express my gratitude towards my co-mentor, Dr. Subhash Chauhan for his tremendous support all these years. They always discussed my research work and provided helpful suggestions which improved my studies. I am extremely grateful to my co-supervisor, Dr. Sheema Khan for her continuous guidance and help all these years.

In addition, I am really grateful to committee members Dr. Santosh Kumar, Dr. Murali Yallapu, Dr. Yi Lu, and Dr. Nadeem Zafar, for their constructive feedback all through my Ph.D studies. Also, I would extend my special gratitude to Dr. Bilal Bin Hafeez for his suggestions and support all the time. Finally, I would thank my family for providing me support and encouragement in both good and bad times. 


\begin{abstract}
Pancreatic cancer ranks as third deadliest cancer worldwide in both men and women. The lack of diagnostic methods, chemo-resistance and invasiveness of the disease diminishes the overall survival rate in patients. Dysregulated glucose metabolism and several molecular determinants including oncogenic signaling pathways play pivotal roles in the development, progression and metastasis of pancreatic cancer. The aberrant glucose metabolism is the most prevalent key factor that influences pancreatic tumorigenesis. The cancer cells of pancreas utilize oxygen for the breakdown of glucose to lactate, which is known as Warburg effect. This phenotype of pancreatic cancer encourages proliferation, growth, migration and invasive phenotypes of cancer cells that worsens the disease. The upstream regulatory molecules responsible for deviant activity of glucose transporters during aberrant glucose metabolism was not very well defined. Thus, identification of new molecular determinants that regulate glucose metabolic pathway would provide promising therapeutic outcomes. Our lab has established the role of MUC13 protein in pancreatic cancer development. Another interesting molecule, Protein Kinase D1 has been reported to play essential role in hypoxic metabolism in squamous cell carcinoma, and glucose uptake in cardiac hypertrophy. Also, it has been extensively studied in our research group, and has been suggested by some other groups to have an essential role in pancreatic cancer. However, its role in regulating glucose metabolism in pancreatic cancer remains elusive. In this dissertation, we investigated that MUC13 and PKD1 proteins are involved in abrupt glucose metabolism in pancreatic cancer cells. We have demonstrated that targeted overexpression of these oncogenes upregulates key oncogenic signaling components involved in aberrant glucose metabolism in pancreatic cancer cells. We also investigated a natural agent Steviol that restored glucose metabolism in pancreatic cancer cells via repression of MUC13 and PKD1 expression.
\end{abstract}

MUC13 is a transmembrane glycoprotein which has an elevated expression in pancreatic cancer. Due to the presence of a cytoplasmic domain, it confers kinase activity and regulates many signaling processes. Therefore, we investigated the effect of MUC13 in metabolic reconditioning of pancreatic cancer. As a result, it was observed that presence of MUC13 in pancreatic cancer upregulated glucose uptake and lactate secretion. This in turn led to more aggressive behavior of the tumor, as evidenced by enhanced proliferation, migration and invasive characteristics of the cells as compared to MUC13 null cells. Interestingly, we observed the interaction of MUC13 with Glut-1 protein, which influences glucose uptake in cancer cells. This was confirmed through various assays such as: immunoprecipitation, immunofluorescence, co-capping and proximity ligation assay. The interpretation of inhibitor results envisaged the role of NFкВ pathway during the molecular interactions of MUC13 and Glut-1. To understand the clinical importance of this mechanism, we evaluated this interaction in tumor samples from the patients with pancreatic disease in advanced stages. Our findings fortified the mechanistic role of MUC13 in rewiring of aberrant glucose metabolism. Altogether, we believed that MUC13 has clinical implication as a key molecule that might be responsible for dysregulated glucose metabolism. 
As discussed earlier about the paucity of associated proteins related to dysregulated glucose metabolism, we further investigated the possible key players with kinase domain for association and activation with other signaling molecules. Protein Kinase D1 (PKD1), a Ser/Thr kinase has been shown to be involved in progression of pancreatic cancer. In this study, we established PKD1 as a novel molecular target and its involvement in regulating aberrant glucose metabolism in pancreatic cancer. We observed that PKD1 was overexpressed in pancreatic cancer tissues compared to normal human tissues. We also found constitutive expression of PKD1 in various pancreatic cancer cells as compared to normal pancreatic ductal epithelial cells. The overexpression of PKD1 in low PKD1 expressing pancreatic cancer cells enhanced tumorigenic characteristics. We observed that specific knockdown of PKD1 inhibited key oncogenic signaling components in pancreatic cancer cells suggesting its oncogenic role in pancreatic cancer. Additionally, during the PKD1 overexpression, cells displayed increased glucose consumption and lactate production. This suggested role of PKD1 in metabolic reprogramming in pancreatic cancer. Also, we observed that PKD1 stimulates the glucose uptake through mTORC1, a component of mTOR signaling pathway. Additionally, the knockdown of PKD1 promoted the chemosensitivity of cells towards gemcitabine and 2DG (2-deoxyglucose). Overall, these results indicated a significant function of PKD1 in rewiring of glucose metabolism in pancreatic cancer.

It was mandating to target and counteract the aberrant glucose metabolism along with the proteins identified in our previous results. In this perspective herein, we proposed steviol, a natural sweetener from plant origin to assess its inhibitory action towards dysregulated glucose metabolism and its associated proteins (MUC13 and PKD1). The reason behind steviol selection for this approach includes that it mimics the glucose molecule that facilitates enhanced uptake of drug (steviol) within the cells and on the other side, it provides glucose homeostasis to adjacent normal cells. These features made steviol as dual functional drug by overtaking other natural drugs for promising results. The exposure of steviol inhibited the proliferative (IC50:10mM) and clonogenic abilities of pancreatic cancer cells. It also suppressed the cell migration and cell invasion capabilities of pancreatic cancer cells. Further, steviol caused the selective inhibition of intracellular glucose intake and lactate accumulation in a concentration dependent manner. The selective inhibition of MUC13 and PKD1 (upstream key proteins of glucose metabolism) that caused the disruption of glucose intake in cancer cells, was observed during steviol treatment. Secondly, steviol interfered with translation-initiation machinery, causing destabilization of the cellular functionality. The impairment of cellular translation process had promoted G1 cell cycle arrest in cells due to the lack of G1-S transition proteins during drug exposure. Collectively, all these events engendered the cellular integrity and directed towards activation of apoptosis.

In conclusion, we evaluated the role of MUC13 and PKD1 in aberrant glucose metabolism leading to growth and aggressive metastatic phenotypes of pancreatic cancer. We also demonstrated efficacy of steviol to repress aberrant glucose metabolism via suppression of MUC13 and PKD1 proteins. 


\section{TABLE OF CONTENTS}

CHAPTER 1. INTRODUCTION .....................................................................................

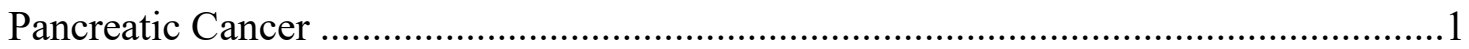

Statistics, Disease Prognosis and Risk Factors ...................................................... 1

Pancreatic Cancer and Tumor Metabolism ................................................................. 1

Glucose Metabolism in Normal versus Cancer Cells ............................................

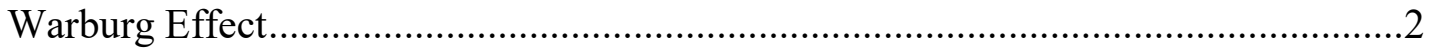

Alterations in Glucose Metabolic Pathways in Pancreatic Cancer .............................2

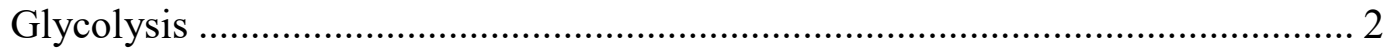

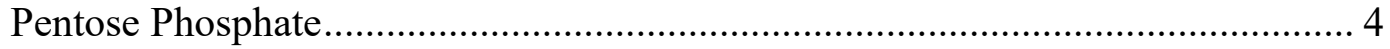

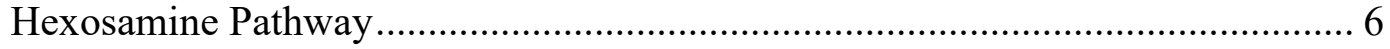

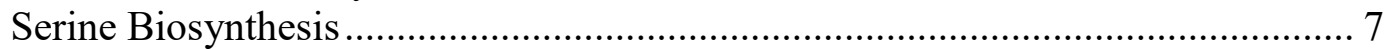

Fatty Acids and Lipid Metabolic Signaling ..................................................... 7

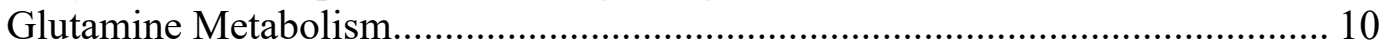

One-Carbon Metabolism Cycle .................................................................... 12

Oncogenic Signaling Implicated in Pancreatic Tumorigenesis ...............................12

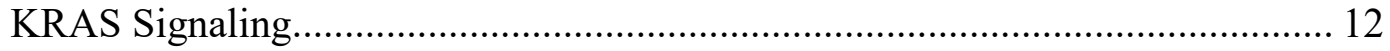

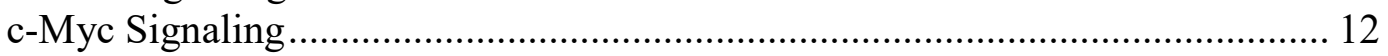

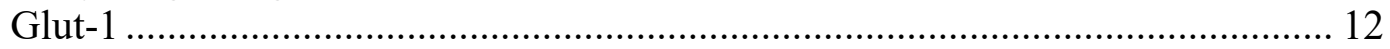

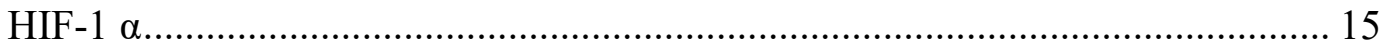

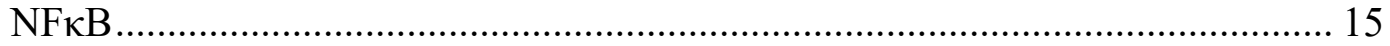

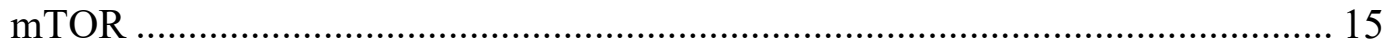

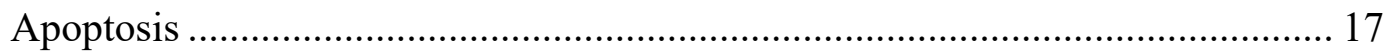

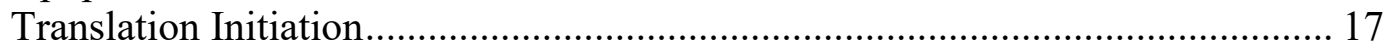

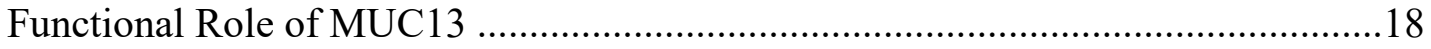

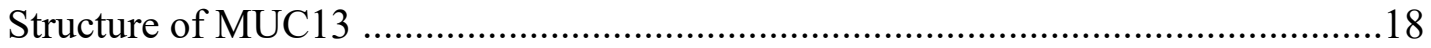

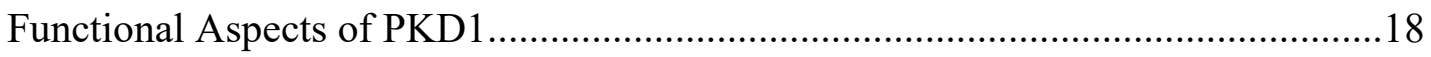

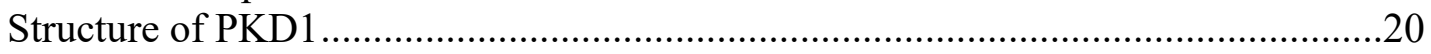

Current Treatment Options for Pancreatic Cancer..............................................20

Molecular Targeting of Glucose Metabolism in Pancreatic Tumors ........................22

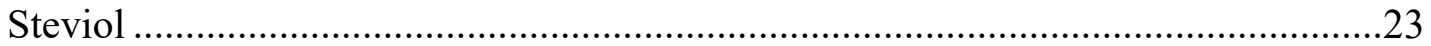

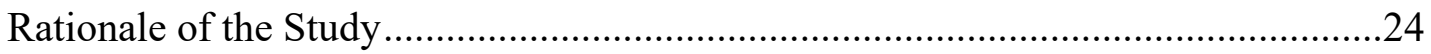

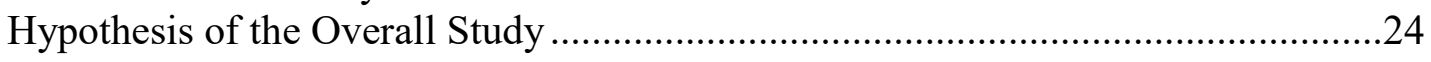

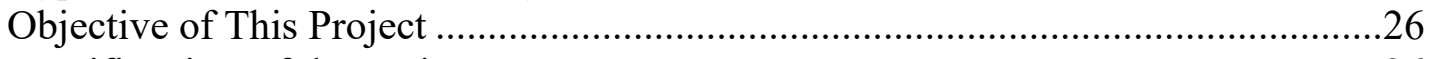

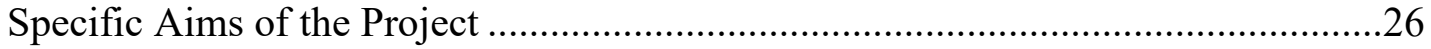

\section{CHAPTER 2. MUC13 CONTRIBUTES TO REWIRING OF GLUCOSE} METABOLISM IN PANCREATIC CANCER ..................................................27

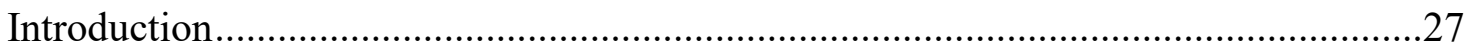

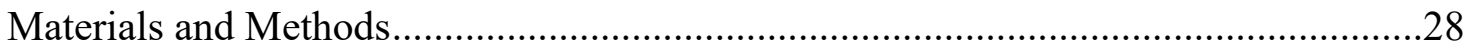

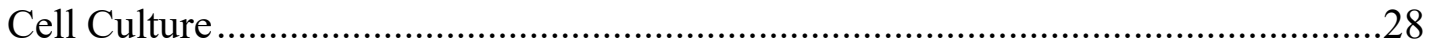

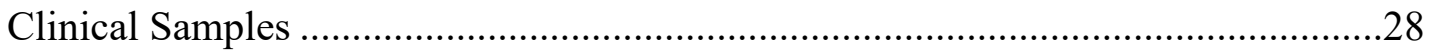

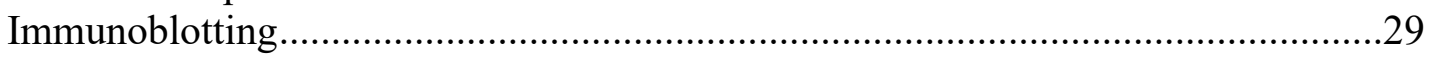

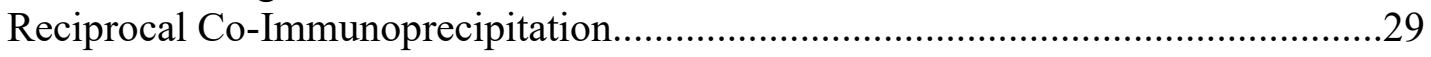


Confocal Immunofluorescence ……………….................................................29

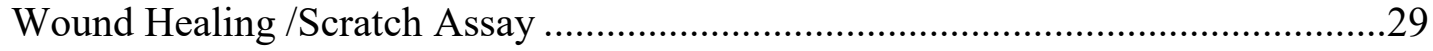

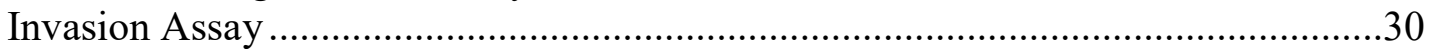

Cell Proliferation Assays ....................................................................................

Lactate and Glucose Assays....................................................................................30

Isolation of RNA and Polymerase Chain Reaction (PCR) ........................................30

In Situ Proximity Ligation Assay (PLA) ……………........................................30

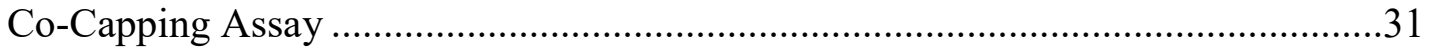

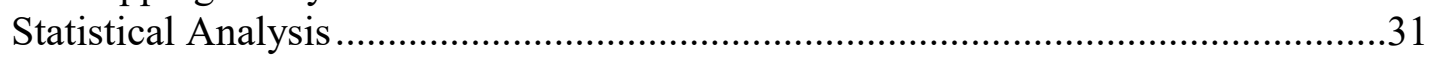

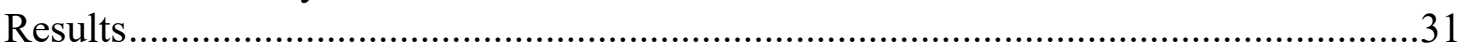

MUC13 Enhances Glucose Metabolism and Invasiveness in PDAC Cells...............31

MUC13 Upregulates c-Myc Expression and the Downstream Effectors of

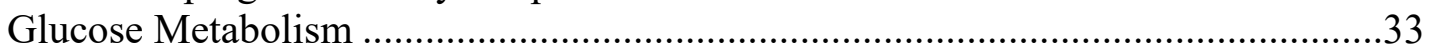

MUC13 Expression Leads to the TNF-Induced Activation/Nuclear Shuttling of

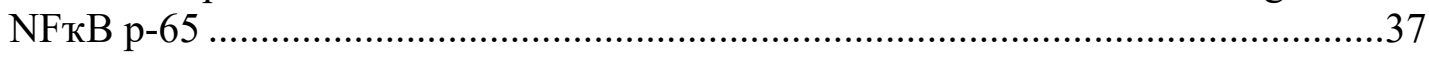

MUC13 Interacts with Glut-1 and This Interaction Is Disrupted by NFкB

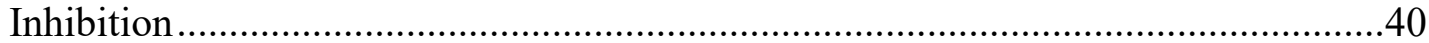

MUC13 and Glut-1 Expression Directly Correlate in Pancreatic Cancer Tissues ....40

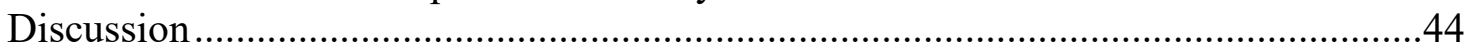

\section{CHAPTER 3. PROTEIN KINASE D1 INDUCES METABOLIC SWITCH IN PANCREATIC CANCER VIA MODULATION OF MAMMALIAN TARGET OF RAPAMYCIN...............................................................................................................46}

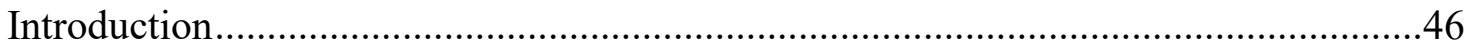

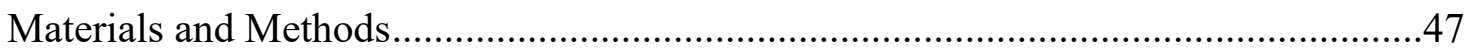

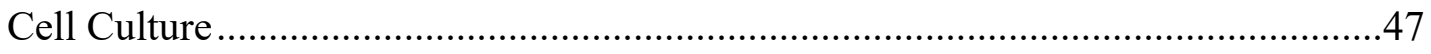

Procurement and Scoring of Human Pancreatic Cancer Tissues for Analysis of

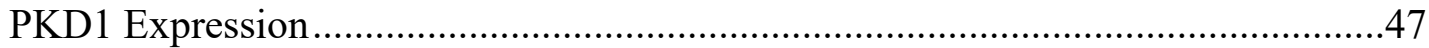

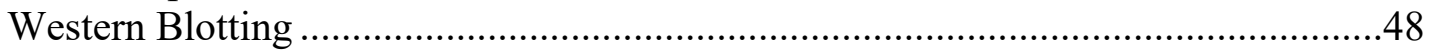

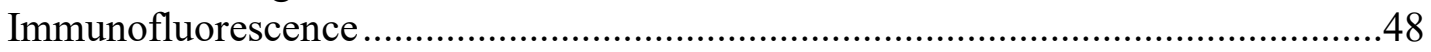

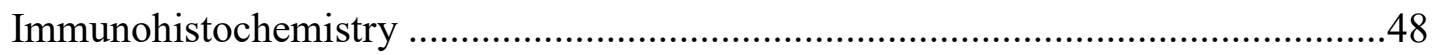

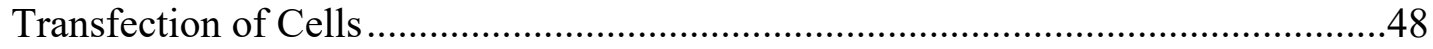

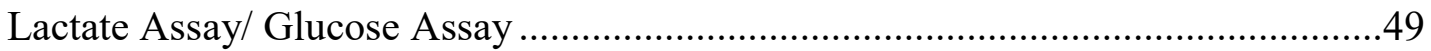

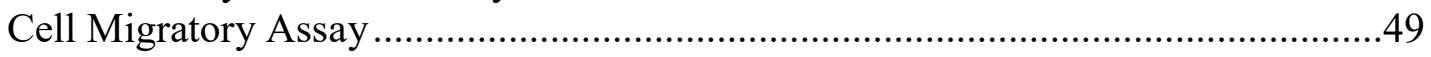

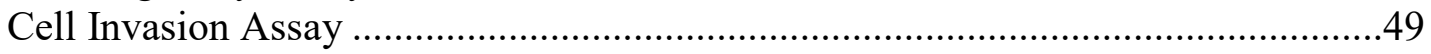

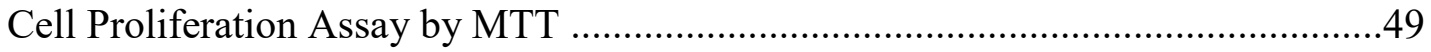

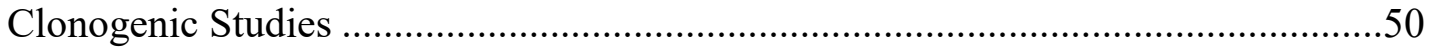

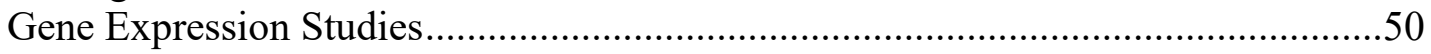

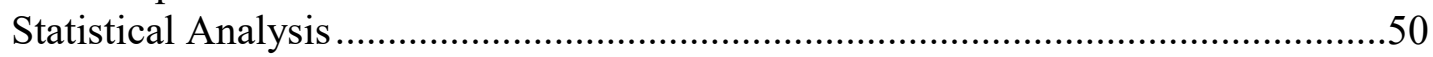

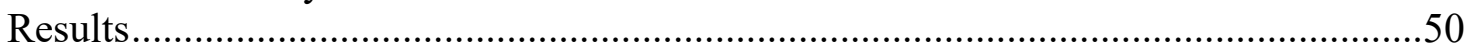

PKD1 Is Overexpressed in Human Pancreatic Cancer Tissues and Cells ..................50

PKD1 Contributes to Tumorigenic Characteristics via Glucose Modulation.............52

PKD1 Expression Leads to Increased Expression of Signaling Proteins That

Trigger Enhanced Glucose Metabolism.....................................................................58

PKD1 Increases Glucose Metabolism via mTOR C1 Activation................................58 
PKD1 Expression Leads to Gemcitabine Resistance and Its Inhibition Sensitizes

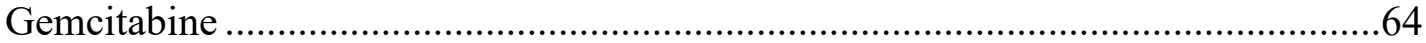

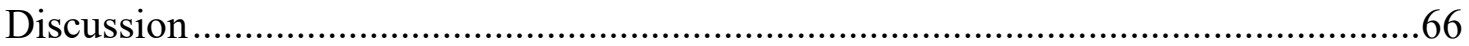

\section{CHAPTER 4. STEVIOL: A NATURAL SWEETENER, INHIBITS PANCREATIC CANCER PROGRESSION THROUGH SUPPRESSION OF ABERRANT GLUCOSE METABOLISM ......................................................................71}

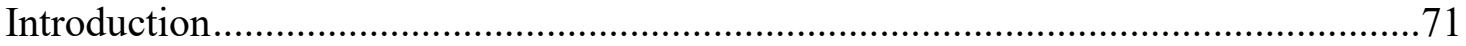

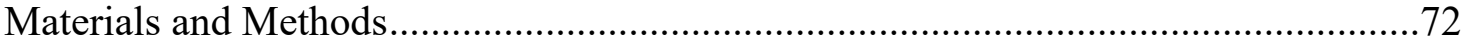

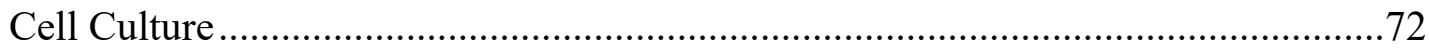

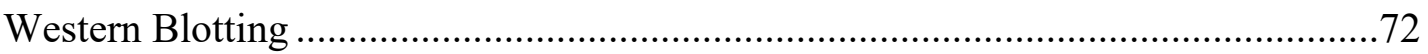

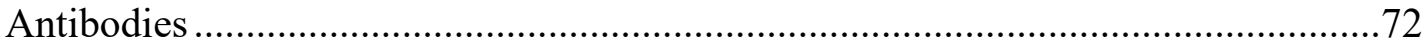

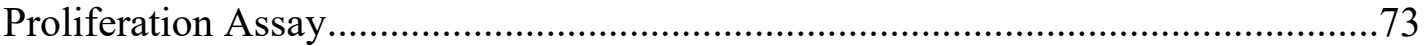

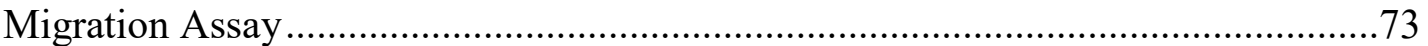

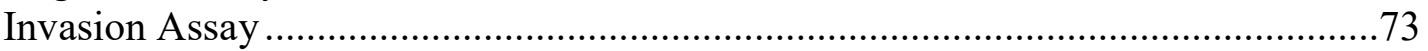

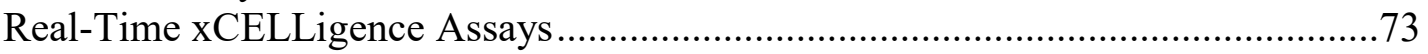

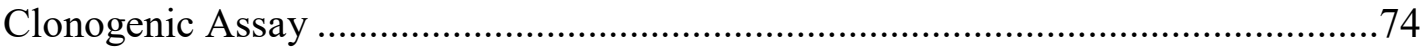

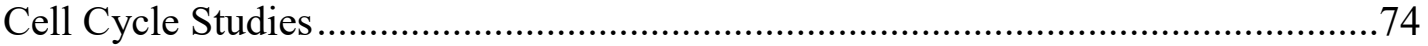

Lactate Assay/ Glucose Assay ............................................................................

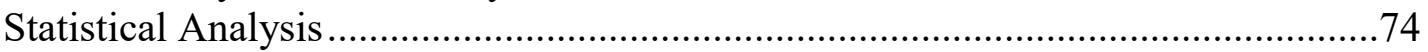

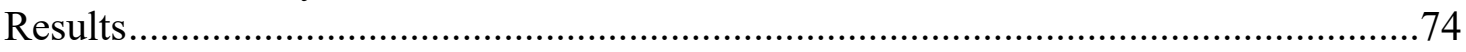

Steviol Effectively Suppressed MUC13 and PKD1 Proteins ....................................74

Steviol Inhibits Cell Proliferation and Colony Formation of PDAC Cells.................75

Steviol Suppresses Cell Migration and Cell Invasion Capabilities of Pancreatic

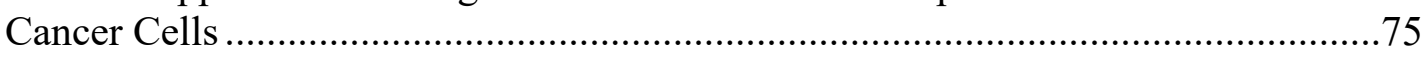

Steviol Suppresses Translation Initiation of Pancreatic Cancer .................................75

Steviol Decreases Glucose Uptake and Lactate Secretion in PDAC Cells.................81

Steviol Induces Apoptosis in Pancreatic Cancer Cells ...............................................81

Steviol Treatment Results in G1 Cell Phase Arrest and Also Regulates the

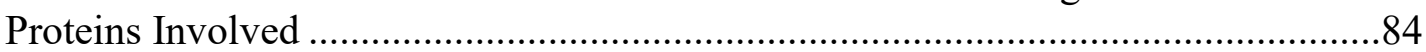

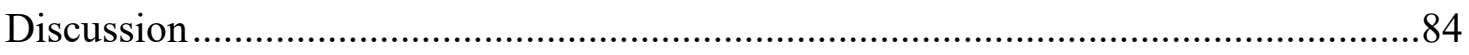

CHAPTER 5. CONCLUSION AND FUTURE DIRECTIONS ....................................88

LIST OF REFERENCES ..............................................................................................93

VITA 


\section{LIST OF FIGURES}

Figure 1-1. Differences Between Normal Cell and Cancer Cell Metabolism ...................3

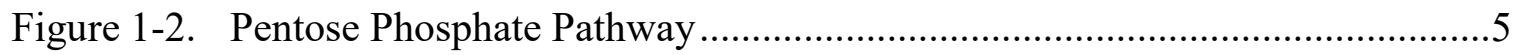

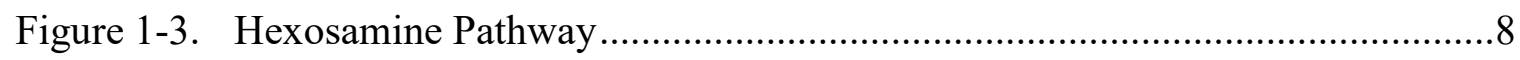

Figure 1-4. Serine Biosynthesis Pathway ...............................................................

Figure 1-5. Fatty Acid and Lipid Metabolism ......................................................... 11

Figure 1-6. Glutamine Metabolic Pathway.................................................................... 11

Figure 1-7. Mechanism of KRAS Signaling............................................................ 13

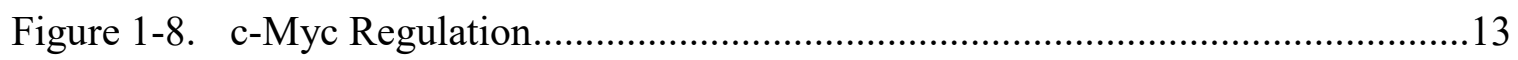

Figure 1-9. Schematic Representation of Function of Glut-1 in Cells ..........................14

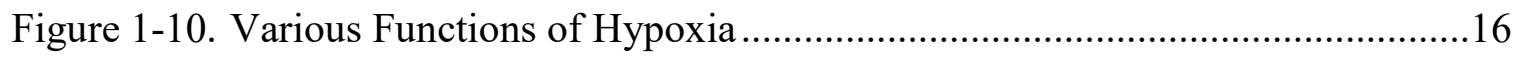

Figure 1-11. Structure of MUC13 Protein ................................................................... 19

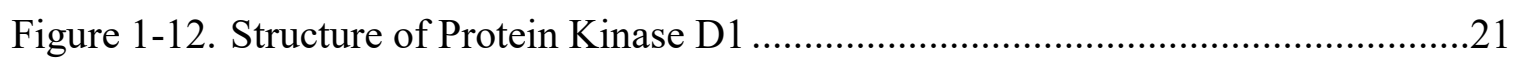

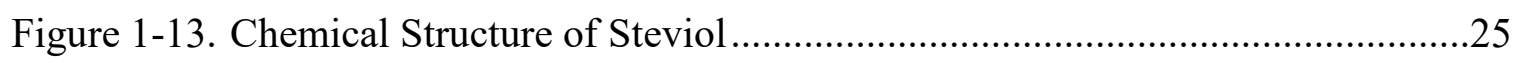

Figure 2-1. MUC13 Enhances Glucose Metabolism in PDAC Cells .............................32

Figure 2-2. MUC13 Regulates Protein and mRNA Expression of Key Factors Involved in Glucose Metabolism ......................................................... 34

Figure 2-3. MUC13 Enhances Expression of c-Myc and Glut-1 in Cells .......................35

Figure 2-4. MUC13 Enhances Expression of HIF-1 $\alpha$ in Cells ......................................36

Figure 2-5. MUC13 Expression Leads to Enhanced Activation/Nuclear Translocation of NFкB p-65 .........................................................38

Figure 2-6. MUC13 Expression Leads to the OA-Induced Activation/Nuclear Translocation of NFKB p-65 ..............................................................39

Figure 2-7. MUC13 Interacts with Glut-1 Protein................................................. 41

Figure 2-8. MUC13-Glut-1 Interaction is Disrupted by Sulfasalazine..........................42 
Figure 2-9. MUC13 and Glut-1 Expression Directly Correlate in Human Pancreatic Cancer Tissues.

Figure 3-1. PKD1 is Highly Expressed in Human Pancreatic Cancer Tissues ...............51

Figure 3-2. Differential Expression of PKD1 in Pancreatic Cancer Cells 53

Figure 3-3. Overexpression of PKD1 Increases Cell Proliferation in Pancreatic Cancer Cells .54

Figure 3-4. PKD1 Enhances Glucose Metabolism in Pancreatic Cancer .55

Figure 3-5. Glucose Metabolism is Enhanced by PKD1 in Pancreatic Cancer Cells......56

Figure 3-6. PKD1 Accelerates Glucose Metabolism in Pancreatic Cancer Cells .57

Figure 3-7. PKD1 Modulates the Expression of Important Proteins Involved in Enhanced Cancer Cell Metabolism

Figure 3-8. PKD1 Elevates the Expression of Associated Genes with Glucose Metabolism.

Figure 3-9. PKD1 Overexpression Increases Cell Proliferation Under Both Normoxic and Hypoxic Conditions.

Figure 3-10. PKD1 Induced Cell Proliferation is Independent of Oxygen Tension

Figure 3-11. PKD1 Enhances Glucose Metabolism Through Activation of AKTmTOR Pathway.

Figure 3-12. Glucose Metabolism is Accelerated by PKD1 Through mTOR Pathway ....65

Figure 3-13. PKD1 Expression Leads to Chemoresistance and its Inhibition Sensitizes Gemcitabine

Figure 3-14. Proposed Mechanism Describing the Role of PKD1 in Impaired Glucose Metabolism in Pancreatic Cancer. .70

Figure 4-1. Steviol Inhibits the Expression of MUC13 and PKD1 Proteins..... .76

Figure 4-2. Steviol Inhibits Cell Proliferation and Clonogenic Potential of Pancreatic Cancer Cells .77

Figure 4-3. Steviol Inhibits Cell Migration and Cell Invasion in Pancreatic Cancer ......78

Figure 4-4. Steviol Causes Reduction in Cell Proliferation and Cell Migration in HPAF-II Cells . .79

Figure 4-5. Steviol Impedes Translation Initiation .80 
Figure 4-6. Steviol Inhibits Lactate Secretion and Glucose Uptake............................82

Figure 4-7. Steviol Inhibits Apoptosis in Pancreatic Cancer Cells ................................83

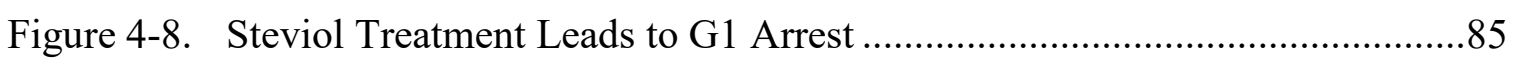

Figure 4-9. Steviol Modulates the Key Proteins Involved in G1 Cell Phase Arrest .......86

Figure 5-1. Schematic Diagram Representing the Overall Conclusion of the Study ......91 


\section{LIST OF ABBREVIATIONS}

$\begin{array}{ll}\text { 2-DG } & \text { 2-Deoxyglucose } \\ \text { DISC } & \text { Death-Inducing Signaling Complex } \\ \text { FADD } & \text { Fas-Associated Death Domain } \\ \text { Glut-1 } & \text { Glucose Transporter-1 } \\ \text { HIF-1 } \alpha & \text { Hypoxia Inducible Factor-1 alpha } \\ \text { KRAS } & \text { Kirsten Rat Sarcoma virus } \\ \text { mTOR } & \text { mammalian Target of Rapamycin } \\ \text { NFkB } & \text { Nuclear Factor-kappa B } \\ \text { PanCa } & \text { Pancreatic Cancer } \\ \text { PDAC } & \text { Pancreatic Ductal Adenocarcinoma } \\ \text { PET } & \text { Positron Emission Tomography } \\ \text { PKD1 } & \text { Protein Kinase D1 } \\ \text { TM } & \text { Transmembrane } \\ \text { TNF } & \text { Tumor Necrosis Factor } \\ \text { TR } & \text { Tandem Repeat }\end{array}$




\section{CHAPTER 1. INTRODUCTION}

\section{Pancreatic Cancer}

Pancreatic cancer is one of the leading causes of cancer-related deaths in the United States, and soon is expected to become the second deadliest type of cancer [1]. Therefore, it is of utmost importance to develop effective strategies to tackle this lethal disease. Pancreatic tumors become highly resistant to the individual chemotherapies. Inaccessible location of the pancreas, late diagnosis, lack of knowledge of molecular determinants, chemoresistance and altered glucose metabolism are the key factors responsible for the aggressiveness of this deadly disease $[2,3]$. In addition to this, several oncogenic signaling pathways play pivotal role in the development, progression and metastasis of pancreatic cancer. Hence, identification of new molecular factors, which play pivotal role in altered glucose metabolism and chemoresistance in pancreatic cancer are urgently required.

\section{Statistics, Disease Prognosis and Risk Factors}

The statistics for pancreatic cancer are extremely alarming. It has been estimated that more than 55,000 people will be newly diagnosed with this disease in the US, including both men and women. This data indicates the severity of this disease [1]. The global sporadic spread of this cancer was profound in Western countries belonging to North America and Europe. This disease accounts to 3\% of the globally occurring cancers with mortality rate of $7 \%$, a major contributor to cancer-related deaths [4]. At the time of diagnosis, $80 \%$ of the patients have advanced metastatic pancreatic cancer [5]. The major risk factors involved in disease progression depend on familial and living lifestyle of the patients that encloses smoking, diabetic positivity, and genetic history [6]. More often, the high rate of pancreatic cancer incidence has been reported in elder people with age of 60 or more, but in few cases, the disease has been diagnosed in young individuals too [7]. Other risk factors include alcohol, obesity, infection (especially with Helicobacter pylori), diet intake and gut microbiome [8].

\section{Pancreatic Cancer and Tumor Metabolism}

Pancreatic tumors are very active metabolically owing to the activation of various oncogenic molecules which aberrantly dysregulate metabolic activities leading to growth, proliferation and drug resistance. The cancer cells have enhanced energy needs to sustain and thus facilitates alterations in constitutive metabolic pathways [9]. It has been reported that pancreatic tumors uptake more fluoro-2-deoxyglucose (FDG) as confirmed by PET (Positron Emission Tomography) [10].

Metabolic reprogramming refers to the increased rate of crucial metabolic processes within the cells during tumorigenic conditions. These include elevated 
glycolysis, pentose phosphate pathway, hexosamine pathway, serine biosynthesis pathway, and lipid and fatty acid metabolism.

\section{Glucose Metabolism in Normal versus Cancer Cells}

Altered glucose metabolism is known to facilitate cancer cell proliferation and survival [11]. The tumor cells highly depend on glucose metabolism for their growth as compared to normal cells. However, the cells with normal constitutive gene expression survive through intake of glucose as energy fuel. These normal cells convert glucose into pyruvate in order to yield energy through glycolysis process for their survival and growth. Subsequently after glycolysis, the initiation of Kreb's cycle promotes the conversion of pyruvate to $\mathrm{CO}_{2}$, and followed by the oxidative phosphorylation leading to the formation of 36 ATP molecules for each glucose molecule. Whereas, in cancer cells, lactate accumulations are predominant that promotes cells towards high rate of proliferation, transforming them towards invasive form, with generation of 2 ATPs [12]. This mechanism is depicted (Figure 1-1). Overall, knowledge on the signaling molecules controlling tumor glucose metabolism is limited which provides a major roadblock for the therapeutic targeting.

\section{Warburg Effect}

Warburg effect is a classical phenomenon discovered by Otto Warburg in 1924, and is widely researched [13]. According to Warburg effect, the cancer cells even under regular oxygen levels, produce abundant amount of lactate. This effect is also referred to as aerobic glycolysis [14]. Under this condition, glucose is consumed and transported to cancer cells, and leading to the excessive lactate accumulations [13]. The production of accumulation of lactate encourages proliferation and growth of cancer cells, which induces an aggressive phenotype. It is the main source of energy requirement for the cancer cells, and thus is a remarkable feature of the tumorigenic phenotype of cells.

\section{Alterations in Glucose Metabolic Pathways in Pancreatic Cancer}

\section{Glycolysis}

Glycolysis is a biological process prevalent in the cancer cells. These cells utilize the several regulatory pathways to achieve their metabolic requirements and also in order to adapt to the tumor microenvironment. This pathway is composed of many reversible steps and three irreversible steps. These steps are also referred as the committed steps. Out of these steps, the first one involves the hexokinases which leads to the generation of glucose-6-phosphate (G6P) from glucose. This step is very significant as glucose molecule is facilitated to enter the cells, and also because it is the branching point for pentose phosphate pathway (PPP), glycogenesis and hexosamine synthesis pathway [15]. 
Quiescent (Normal) cells

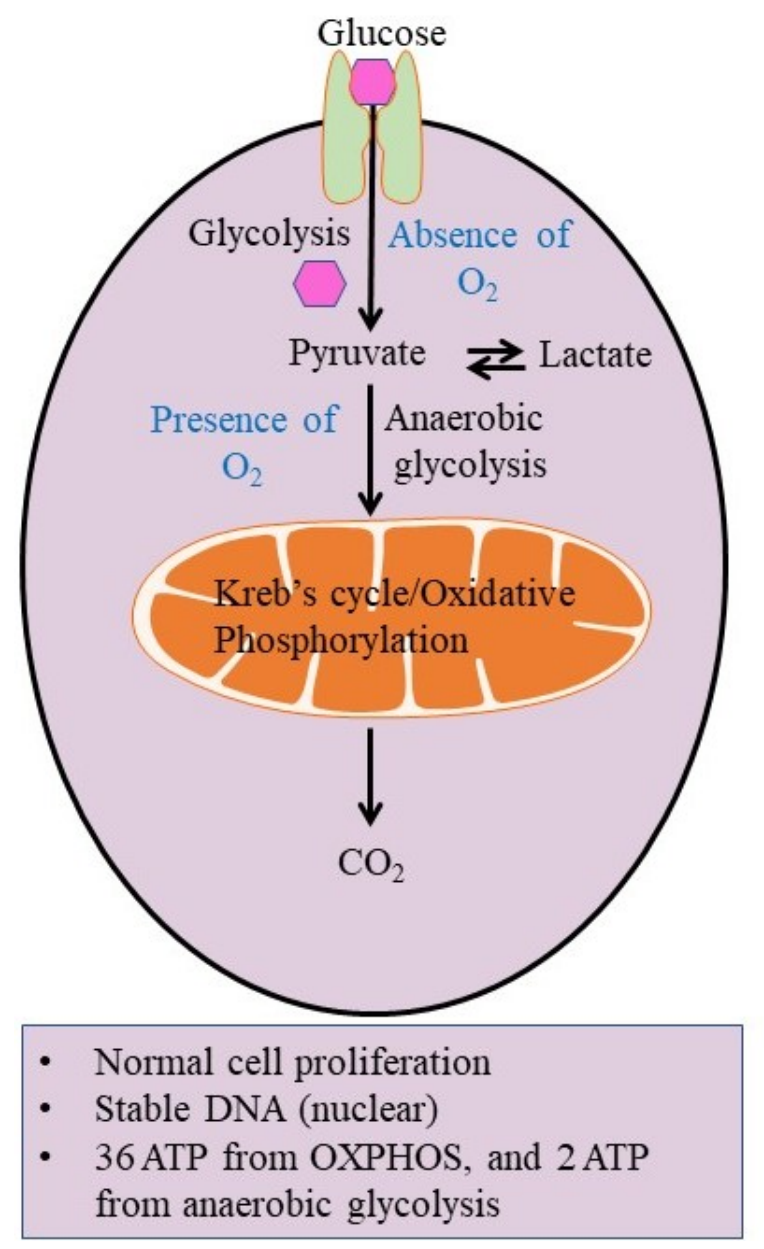

Proliferating (Cancer) cells

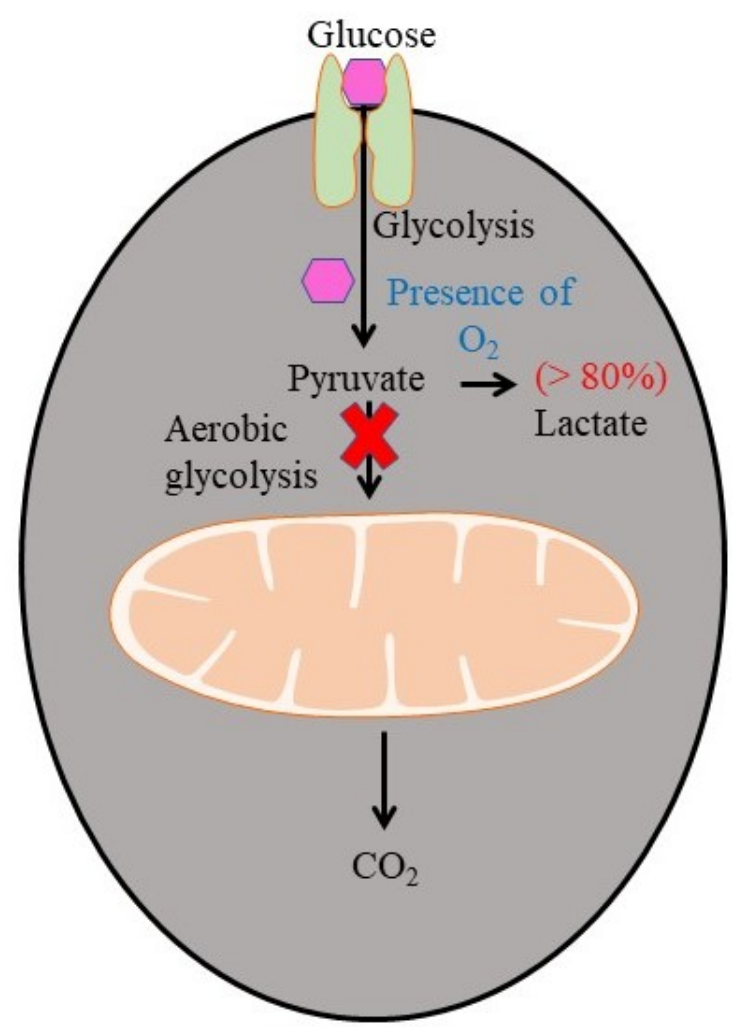

- Uncontrolled cell proliferation

- Unstable nuclear DNA

- Production of 2 ATP molecules from aerobic glycolysis

\section{Figure 1-1. Differences Between Normal Cell and Cancer Cell Metabolism}

The cancer cells are more metabolically active compared to the normal cells due to the enhanced glycolytic flux, ultimately resulting in increased cell proliferation and tumorigenic features. 
Lactate is an end product of glycolysis, and was not an attractive target until few years. But in recent years, it has evolved as a promising target for cancer metabolism. It has been reported that increased levels of lactate are directly proportional to enhanced cancer cell proliferation, growth, and metastasis. They transport lactate to proximal cancer cells, stroma and other regions such as the endothelial cells, which leads to metabolic alterations. It also promotes angiogenesis of tumors. Therefore, it is considered as an essential target for cancer therapeutics nowadays. Lactate dehydrogenase A (LDHA) is a critical enzyme involved in cancer cell metabolism. The other isoform is lactate dehydrogenase B (LDHB). Precisely, LDHA is the mainly expressed isozyme in cancer cells. Both these isozymes are capable of forming homo- or hetero-tetramers [16, 17]. Increased levels of LDHA is associated with tumor progression involving high rates of glycolysis, and makes the prognosis of the disease extremely difficult [18]. Also, many genetic studies have indicated that targeting this enzyme can be extremely useful for cancer therapy.

The cancer cells export lactate, instead of utilizing them as an energy source. This leads to acidification of the tumor microenvironment [19]. Monocarboxylate transporters (MCT) are used to transport lactate and protons under high glycolytic conditions. Therefore, they can control the $\mathrm{pH}$ and lactate concentration in cell. There are four isoforms of MCT transporters- MCT1, MCT2, MCT3 and MCT4 [20]. Of these, high levels of MCT1 and MCT4 indicate tumorigenic condition and poor prognosis in different types of cancer [21-26]. But their role in pancreatic cancer is still unknown.

It has been reported that the transcription factor Forkhead box protein M1 (FOXM1) enhances aerobic glycolysis (Warburg effect), and induces lactate dehydrogenase A (LDHA) expression, thereby leading to progression of pancreatic cancer. Therefore, targeting the FOXM1-LDHA signaling pathway can be useful for pancreatic cancer treatment [27].

\section{Pentose Phosphate}

Pentose phosphate pathway branches out from glycolysis and plays an essential role in fulfilling the anabolic requirements of cancer cells, and also to inhibit the oxidative stress of the cells. This pathway has both oxidative, as well as non-oxidative mechanisms. The oxidative arm mainly leads to production of NADPH and ribulose-5phosphate, mainly in three different irreversible steps. The non-oxidative arm of this pathway produces pentose phosphates, such as ribose 5-phosphate, which is essential for synthesis of nucleic acids and further leading to increased cell proliferation. Some other molecules are also involved in these processes such as fructose-6-phosphate and glyceraldehyde-3-phosphate[28, 29]. This overall pathway is illustrated in Figure 1-2.

As this pathway generates NADPH, which is utilized for the production of fatty acids and also for pentose phosphate molecules, it has a significant role in tumor cell metabolism. The level of cellular metabolism and redox potential determines the 


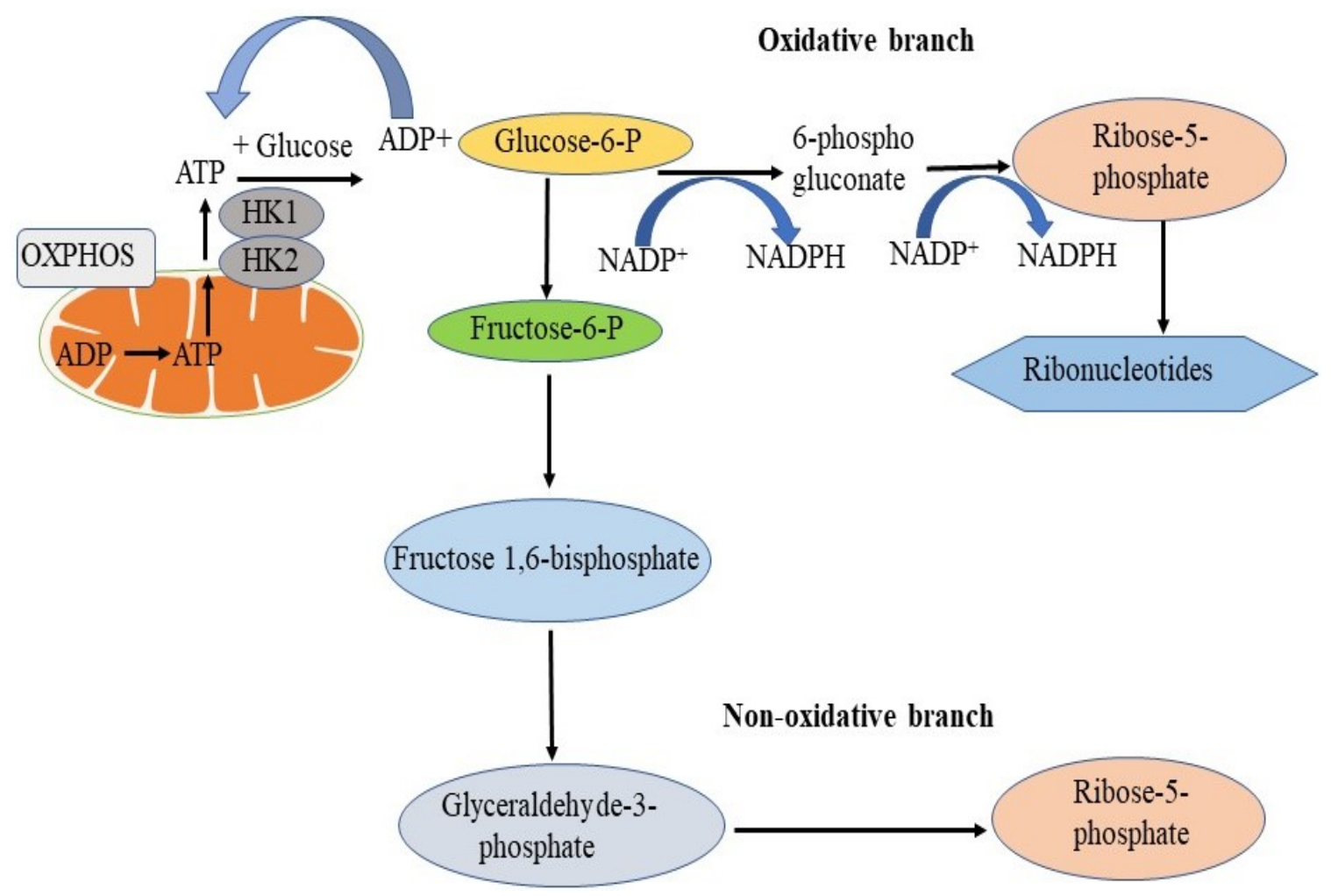

Figure 1-2. Pentose Phosphate Pathway

The enzymes hexokinase 1 (HK1) and hexokinase 2 (HK2) generate glucose 6-phosphate from glucose by utilizing the ATP molecule produced during oxidative phosphorylation (OXPHOS). 
oxidative and non-oxidative mechanisms. This pathway is regulated by many agents, and in cancer cells both mechanisms (oxidative and non-oxidative) of this pathway are accelerated by various oncogenes $[28,29]$. The tumor cells mainly utilize the nonoxidative branch of this pathway to produce nucleotides for RNA/DNA generation. This has been reported in some study that about $80 \%$ of the nucleotides are obtained through the nonoxidative arm of this pathway [30].

The most important role of the PPP in the context of carcinogenesis is its protective role from cell death. Recently, it has been identified that the emerging lesions of cancer cells can regulate the fluctuations of this pathway. The enzymes which are involved in this pathway are very critical in regulating the rate and the functioning of this pathway. This is led by various mechanisms which determine the regulation and activities of these enzymes. The activation of glucose-6-phosphate dehydrogenase (G6PDH) is mediated by activation of various pathways such as PI3K/Akt and Ras. The increased rate of PPP in proliferating cells is one of the distinguishing factor from quiescent cells. It has been reported that the occurrence of drug resistance occurs due to the increased levels of PPP in various cancers. Also, oxidative stress [31], radiations [32] and some other chemotherapeutic agents [33] contribute towards the elevated levels of this pathway, which further results in increased ROS generation.

The increased levels of G6PDH are indicators of high oxidative mechanism in this pathway, after the occurrence of drug resistance. But this resistance can be overcome by some agents such as DHEA or 6-AN, as reported in colon cancer. These molecules are capable of restraining the first two steps of the oxidative branch of the PPP [34]. During dysregulated condition of this pathway, increased growth and proliferation of cancer occurs. Proper understanding of this pathway and its correlation with glycolysis can enable the emergence of effective therapeutic targeting.

\section{Hexosamine Pathway}

Hexosamine pathway branches from glucose metabolism and is initiated by glucose- 6 phosphate and fructose- 6 phosphate. It has been reported that this pathway has a significant role in tumor progression and insulin resistance $[35,36]$. About $5 \%$ of glucose is utilized in this pathway which is then converted to fructose-6-phosphate (F6P) in two steps. Further, the enzyme glutamine:fructose-6-phosphate amidotransferase (GFAT1) catalyzes the rate-limiting step of this pathway. The amide group on glutamine gets transferred to F6P leading to the formation of GlcN-6-P, and this reaction is catalyzed by GFAT1. The pancreatic cancer cell lines are very responsive to glutamine starved conditions. Therefore, GFAT1 enzyme is able to combine the glucose as well as glutamine metabolism, and leads to tumor progression. In a study, it was found that elevated level of this enzyme results in poor prognosis of patients suffering from pancreatic cancer [37]. In another study, it was reported that the genetic variations of this enzyme can lead to risks of pancreatic cancer development [38]. The end product of hexosamine pathway, UDP-N-acetylglucosamine (UDP-GlcNAc) is involved in the post- 
translational modification of several proteins through $\mathrm{O}$-linked $\beta$-Nacetylglucosamine $(\mathrm{O}-$ GlcNAc). This Hyper-O-GlcNAcylation mechanism is anti-apoptotic and results in activation of NFאB pathway in PDAC [39]. In another study, the researchers found that by altering the $\mathrm{Sp} 1$ (transcription factor regulating cancer cell survival and proliferation) modification mechanism through O-GlcNAc, pancreatic cancer cell death occurs through triptolide, which is undergoing phase I clinical trials [40]. Glycogen is produced during this process, and can act as an alternative for glucose when it is limited for cancer cells [41]. This mechanism is shown in illustration (Figure 1-3).

\section{Serine Biosynthesis}

The serine biosynthesis pathway (Figure 1-4) involves the production of 3phospohoglycerate (3PG) during glycolysis to form some non-essential amino acids. The enzyme phosphoglycerate dehydrogenase $(\mathrm{PHGDH})$ is inhibited in certain cancer cells. The initial step in this pathway is catalyzed by PHGDH, leading to the formation of 3phosphohydroxypyruvate. Inhibitors of this enzyme have recently been reported, and it was found that they inhibited the growth and proliferation of cancer cells which had increased rate of serine biosynthesis. Few of these inhibitors are currently undergoing clinical studies and some are approved for therapies as well [15]. PHGDH is required for the proliferation of cancer cells with or without the availability of serine. Therefore, it is unclear what leads the cancer cells to increase the activity of the initial steps of this pathway. Excessive amount of serine accounts for increased glycolysis rate leading to tumorigenesis [42].

Liver kinase B1 (LKB1) is a known tumor suppressor. In a recent study, it was observed that loss of LKB1 is linked to serine metabolism in pancreatic cancer, and leads to DNA methylation and tumor progression. The researchers found that due to activation of the serine-glycine-one-carbon pathway, in conjunction with S-adenosylmethionine formation, through mTOR, there was increased correlation between loss of LKB1 and activation of KRAS [43]. In another study, the researchers investigated if the uptake of fluorine-18 fluorodeoxyglucose (FDG) has any association with mTOR signaling pathway. They observed that the expression levels of Glut-1, p70S6 and EGFR were correlated with FDG uptake, and this in turn was associated with glucose metabolism and tumorigenesis in pancreatic cancer patients [44].

\section{Fatty Acids and Lipid Metabolic Signaling}

Fatty acids serve as an energy source for tumor cells, and act as precursors for catabolism in cancers. Their metabolism is modulated by 5'-AMP-activated protein kinase (AMPK), which gets activated when less ATP is produced. Increased levels of fatty acids are one of the important factors for aberrations in the metabolic pathways of cancer cells. It leads to increased cancer cell proliferation and survival, and the knockdown of essential enzymes associated with fatty aid metabolism enables the retardation of cell growth and survival [45]. 


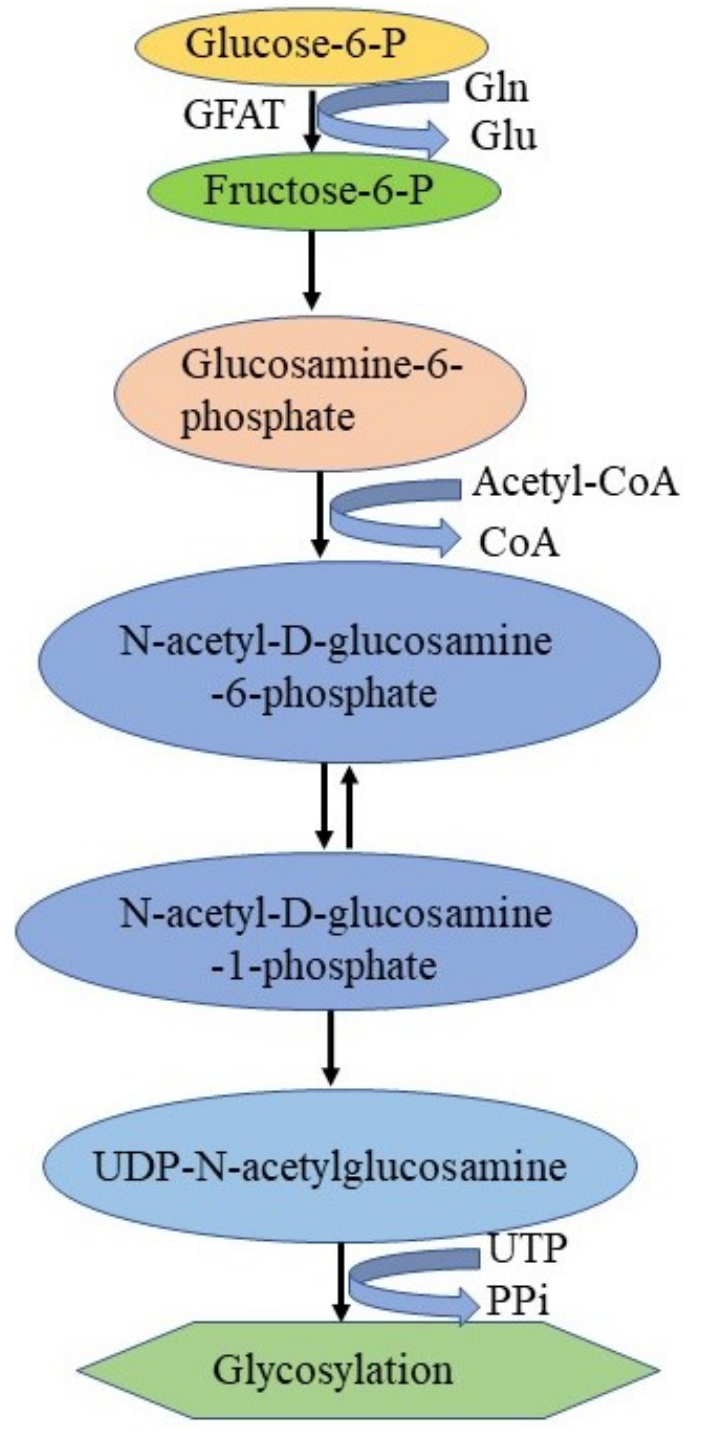

Figure 1-3. Hexosamine Pathway

This is an intermediate pathway which is essential for the production of glycosylation molecules. 


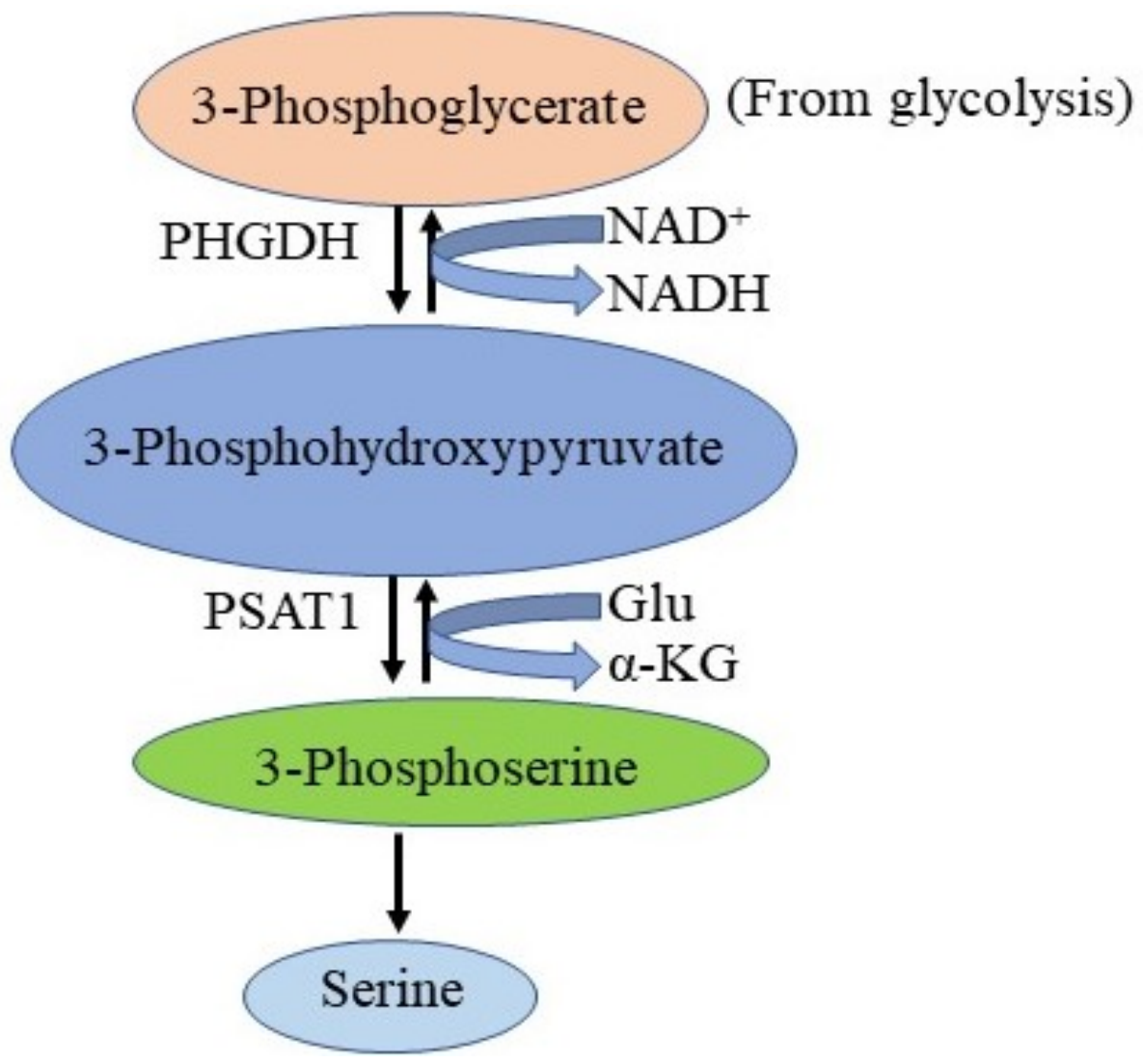

Figure 1-4. Serine Biosynthesis Pathway

This pathway uses the 3-phosphoglycerate produced during glycolysis to generate 3phosphohydroxypyruvate. In the following step, 3-phosphoserine is produced, which is later converted to serine. This molecule (serine) is consumed during folate metabolism and methionine cycle. 
In a recent research, it was found that the serum fatty acid synthase (FASN) had high levels and it was considered as an indicator of neoplasia. Based on the data, it showed a potential for a therapeutic target. In some other research, it was found that some PDAC cells are able to get fatty acids through circulation, liver, adipose tissues for their proliferation [45]. These correlations can be exploited in the future for evaluation of new therapeutic targeting strategies for pancreatic cancer.

The cancer cells require fatty acids for their proliferation. In another study, it was found that by inhibiting fatty acid synthesis, the pancreatic cancer cells undergo more apoptosis. In this study, an inhibitor of lipid synthesis: 5-(tetradecyloxy)-2-furoic acid (TOFA) was used. It inhibited AC carboxylase (ACC). Further, they also assessed the efficacy (in inhibiting the proliferative abilities of pancreatic cancer cells) of fatty acid synthase inhibitors: cerulenin and irgasan. It was observed that these inhibitors markedly decreased the proliferation of the pancreatic cancer cells [46]. Some researchers have also studied the role of fatty acids and metabolic pathways (mevalonate) towards the growth and proliferation of pancreatic cancer stem cells [47]. The fatty acid/lipid metabolism process is illustrated through Figure 1-5.

\section{Glutamine Metabolism}

Glutamine is an amino acid which is highly used by the cancer cells to fuel anabolic metabolism processes. This nutrient is used mainly for cellular growth, and through the Kreb's cycle, it can be metabolized to generate energy. It also contributes to synthesis of fatty acids in cells under hypoxic conditions. In case of lipid synthesis, it serves as a carbon donor instead of glucose. Also, it is critical for glutathione production, which is essential for growth and proliferation of tumor cells during oxidative stress and depicted in Figure 1-6. In PDAC, a different role of glutamine is to transaminate glutamate and oxaloacetate into $\alpha$-ketoglutarate and aspartate. In a study, the authors have identified that oncogenic KRAS plays an important role in altering glutamine metabolism. Therefore, targeting this pathway can provide effective therapeutic strategies for PDAC patients [48]. Researchers have found that by transfer of glutamine, adipocytes increase cell proliferation in pancreatic cancer [49]. Research has shown that glutamine anaplerosis decreases growth and proliferation of pancreatic cancer by inhibition of autophagy [50]. In normal cells, glutamate dehydrogenase (GLUD1) is used in the mitochondria for the conversion of glutamate into $\alpha$-ketoglutarate during the TCA cycle. But in PDAC, aspartate gets converted into oxaloacetate through aspartate transaminase (GOT1), which takes place in the cytoplasm. Further, the oxaloacetate gets converted to malate and later to pyruvate. This increases the NADPH/NADP $(+)$ ratio and leads to the maintenance of redox state in the cells. The researchers established that oncogenic KRAS is strongly involved in the metabolic reprogramming of glutamine, and therefore targeting this pathway can provide novel therapeutic strategies for the treatment of these deadly tumors [48]. 


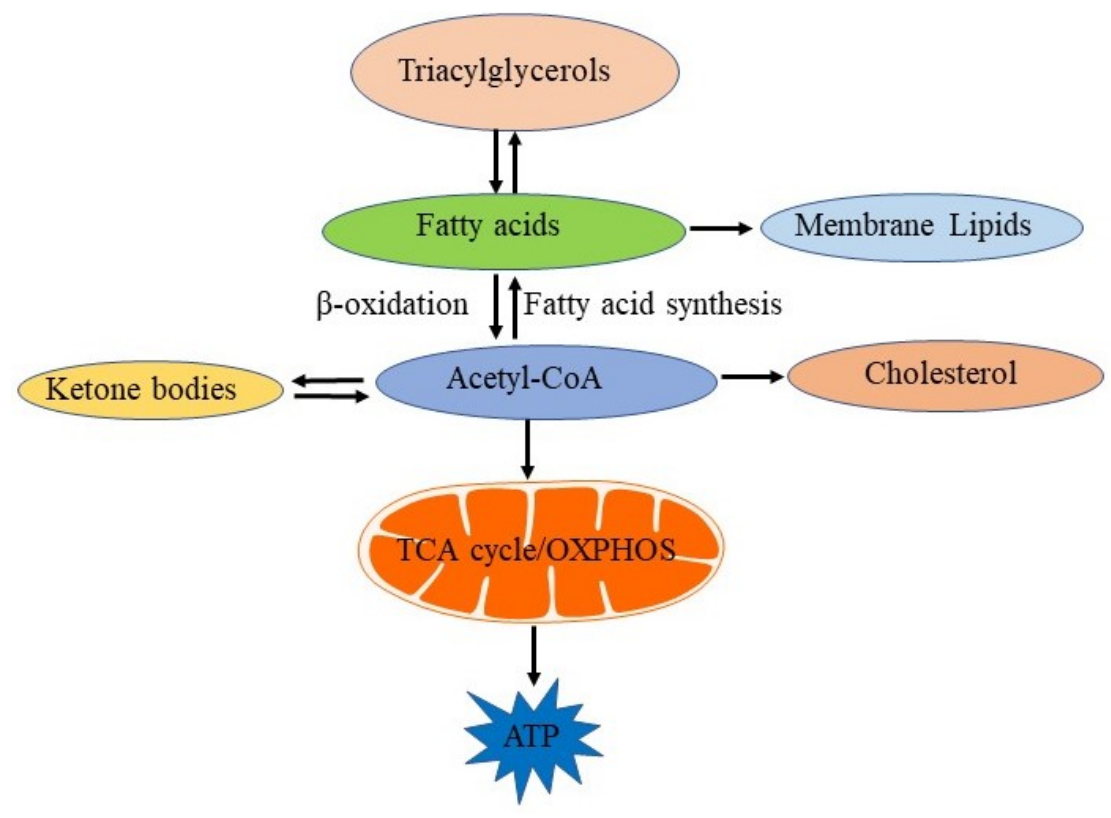

\section{Figure 1-5. Fatty Acid and Lipid Metabolism}

Elevated levels of fatty acids and lipids, both account for metabolic aberrations in cancer. This further leads to increased cell proliferation and metastatic characteristics in cancer cells.
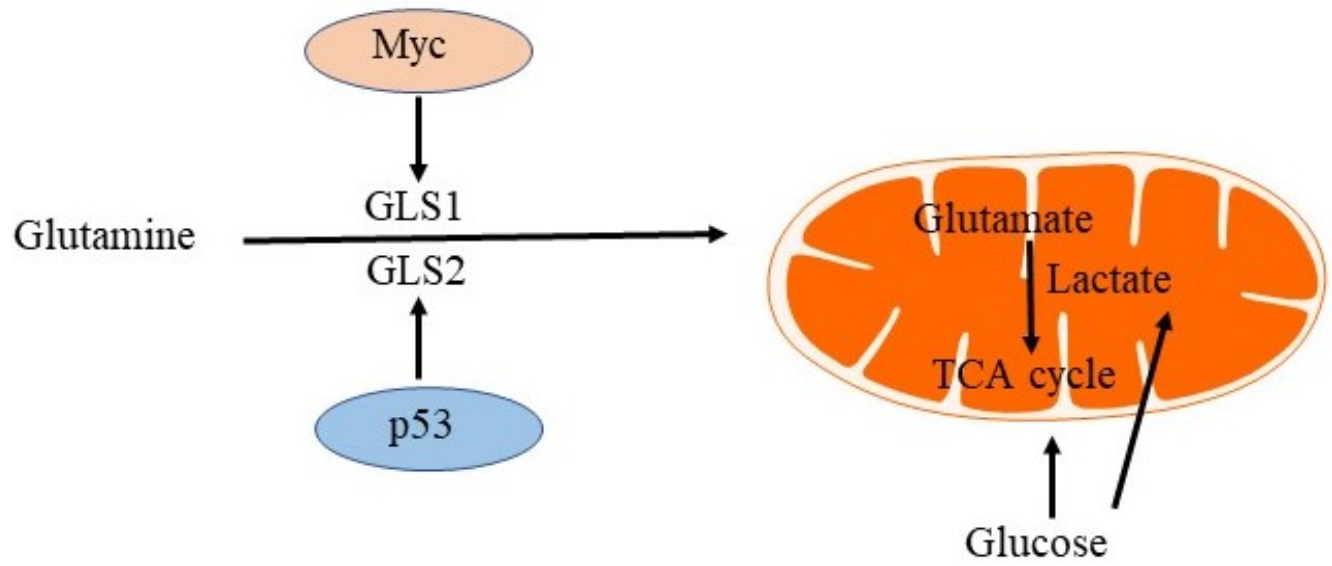

Figure 1-6. Glutamine Metabolic Pathway

This pathway is also utilized by cancer cells for their rapid growth and to obtain energy. GLS1 and GLS2 refers to glutamine synthase 1 and glutamine synthase- 2 enzymes respectively. 


\section{One-Carbon Metabolism Cycle}

Serine and glycine are responsible for one-carbon metabolism to form nucleotides. This cycle generates precursor molecules such as NADPH for other pathways such as methionine and purine synthesis cycle, and is also involved in methylation. For this, transfer of carbon groups takes place and the reactions are facilitated by various enzymes and intermediates. Many inhibitors for the enzymes for this pathway have been identified and are currently under clinical trials [51].

\section{Oncogenic Signaling Implicated in Pancreatic Tumorigenesis}

\section{KRAS Signaling}

KRAS signaling pathway is the most prevalent pathway involved in pancreatic cancer. About $95 \%$ of pancreatic tumors harbor KRAS mutation [52]. It is well studied that oncogenic form of KRAS is involved in metabolic alterations of cancer cells [53]. KRAS mutations also serve as an important factor in the growth, progression and resistance mechanisms of pancreatic cancer [54]. This molecular signaling mechanism also upregulates proliferation of cells, migratory potential and autophagy in cells [55]. A schematic depicting KRAS signaling with respect to glucose metabolism is illustrated in Figure 1-7.

\section{c-Myc Signaling}

c-Myc is an oncogenic molecule which regulates cell growth and metabolism. Due to its involvement in various associated processes, it is considered as a "master regulator" [56]. It is also a crucial transcriptional factor associated with cancer. [57]. Studies have suggested that c-Myc is associated with glycolysis [58]. It is also involved in Warburg effect, as it increases the expression level of associated genes in this process [59]. Various studies have indicated that c-Myc is associated with pancreatic carcinogenesis, and therefore its suppression can have beneficial therapeutic outcomes for PDAC [60]. It performs a plethora of functions as depicted in Figure 1-8.

\section{Glut-1}

Glut-1 stands for Glucose transporter-1 and belongs to the GLUT family. It facilitates the uptake of glucose within the cells. A schematic depicting the role of Glut-1 is shown in Figure 1-9. Glut-1 is present in endothelial cells, red blood cells, blood-brain barrier regions as well as in placenta $[61,62]$. Its overexpression leads to high glucose metabolism [63]. Many reports have suggested that overexpression of Glut-1 directly correlates with increased tumorigenic features, and thus it can be used as a biomarker for 


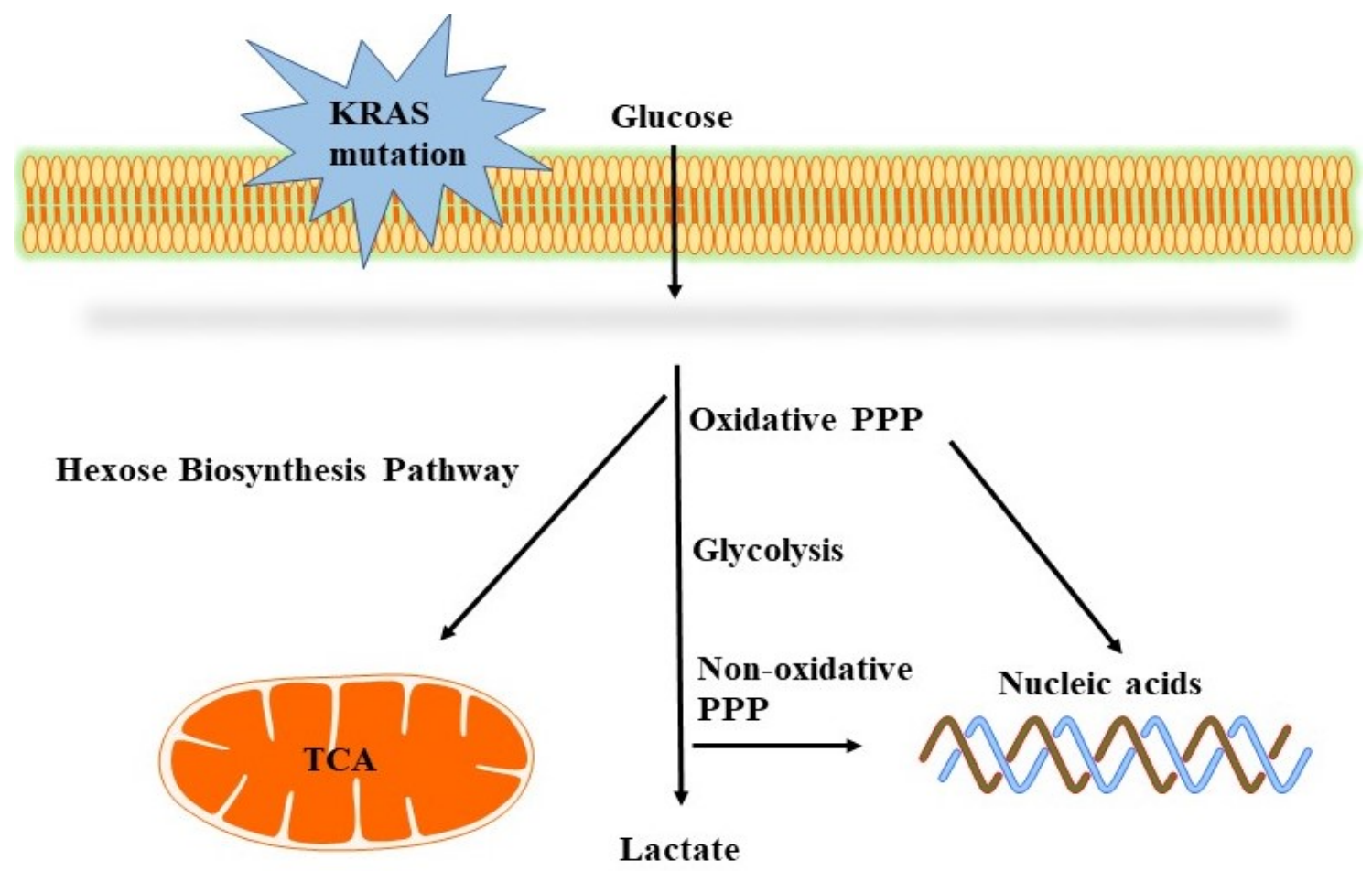

Figure 1-7. Mechanism of KRAS Signaling

This molecular pathway is predominant in pancreatic cancer, and is also closely associated with aberrant glucose metabolism.

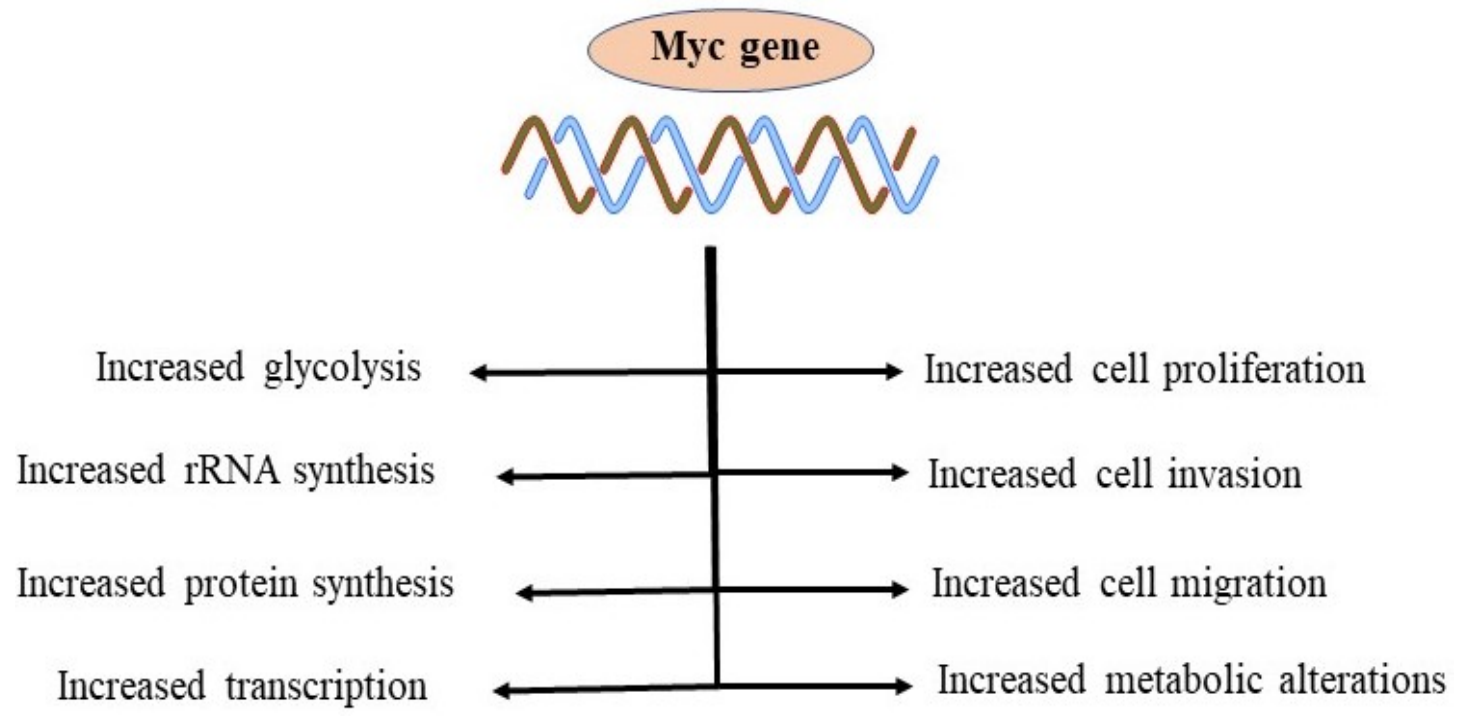

Figure 1-8. c-Myc Regulation

This oncoprotein is involved in various cellular functions as depicted in this figure. 


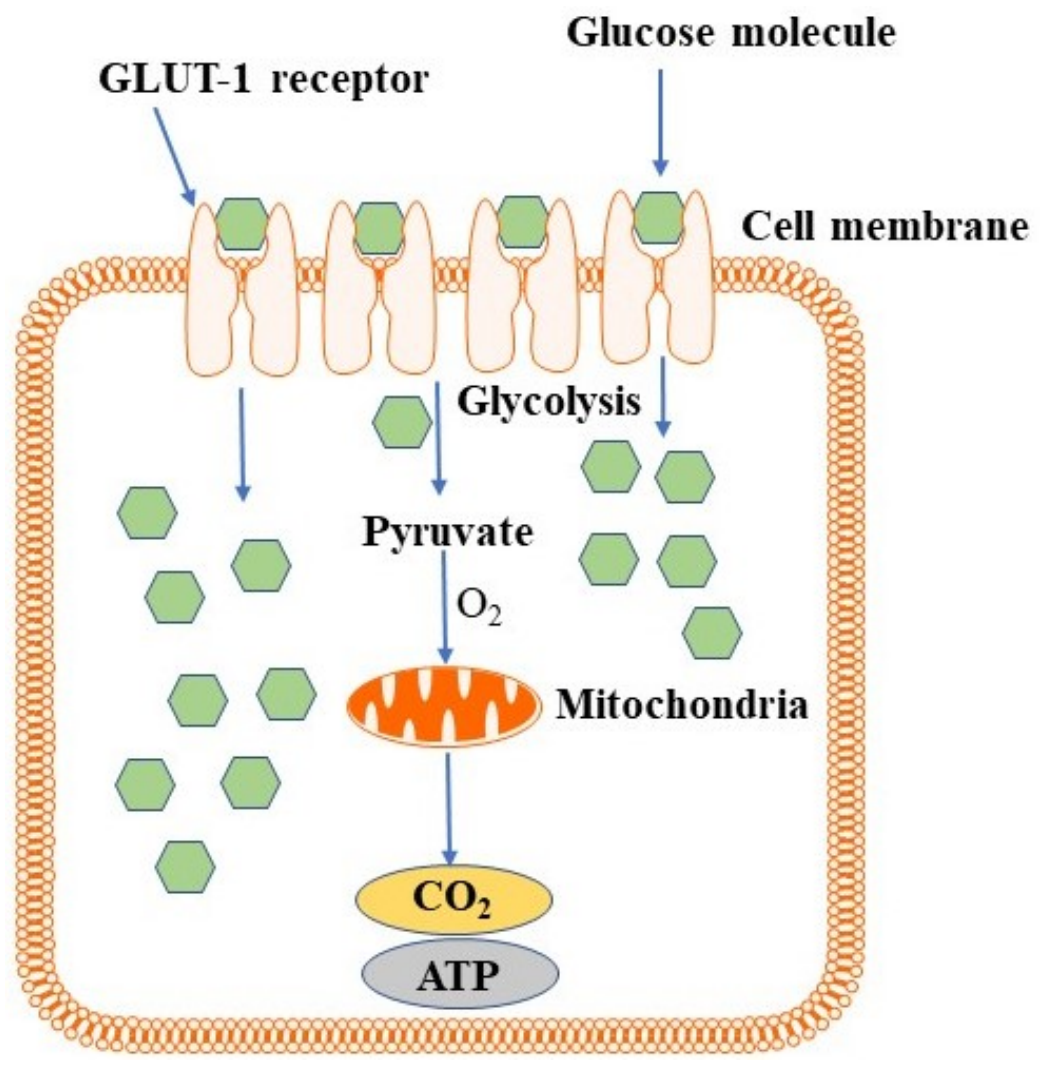

Figure 1-9. Schematic Representation of Function of Glut-1 in Cells

Glucose is taken inside the cells when glucose molecule binds to the Glut-1 receptor on the cell membrane and is later released within the cell. The glucose molecule is further used by the cells to generate energy in the form of ATP. (Original individual figure components were made using Motifolio software). 
pancreatic cancer. The other glucose transporters are GLUT2, GLUT3 and GLUT4, but GLUT1 is the prominent one [61].

Of all the glucose transporters, Glut-1 is believed to be involved in the consumption of FDG (fluorodeoxyglucose), which is a method to detect hypoxic condition within the tumors, through PET (positron emission tomography) technique [64]. Compelling evidences indicate Glut-1 can be a prognostic marker as well as a therapeutic molecule to target pancreatic carcinogenesis $[65,66]$.

\section{HIF-1 $\alpha$}

HIF-1 $\alpha$ stands for Hypoxia-inducible factor $\alpha$. It is an important transcription factor which helps tumor cells to survive under hypoxia. It enhances tumorigenic condition by increasing the expression of genes associated with metabolism. It is a key molecule which regulates cancer metabolism through stimulation of essential genes involved in glycolytic pathway.

Hypoxia inducible factors are formed of two parts: $\alpha$ - subunit, and a $\beta$-subunit. The alpha $(\alpha)$ portion is sensitive to oxygen. HIF-1 $\alpha$ is a key molecule which controls the oxygen homeostasis in cells, and in turn functions in various pathophysiological conditions, growth and development $[67,68]$. Under hypoxic conditions, the genome becomes very unstable and repair of DNA gets ineffective. This in turn leads to a tumorigenic condition [69]. The various functions of HIF-1 $\alpha$ are illustrated in Figure 1-10.

\section{NFкB}

$\mathrm{NF \kappa B}$ is a principal regulator involved in various pathological conditions such as: cancer, autoimmune diseases and inflammation. It plays a key function in the metabolic adaptation of both normal and tumor cells, and also controls mitochondrial respiration [54].

The NFאB complex includes the $\mathrm{p} 65$ protein, the $\mathrm{p} 50$ protein and the inhibitory $\mathrm{I} \kappa \mathrm{B}$ kinase (IKK) protein. In inactive state, NFאB protein is present in the cytoplasm, but in the activated state it translocates to the nucleus [70]. NFKB protein is associated with tumor growth and subsequent progression. Therefore, several studies have been conducted to exploit this pathway as a potential therapeutic strategy to target various cancers, including pancreatic cancer.

\section{mTOR}

The mammalian target of rapamycin (mTOR) is a prime player of the PI3K signaling. It is involved in several aspects such as: cellular growth and proliferation with respect to the energy level and nutrient source [71]. 

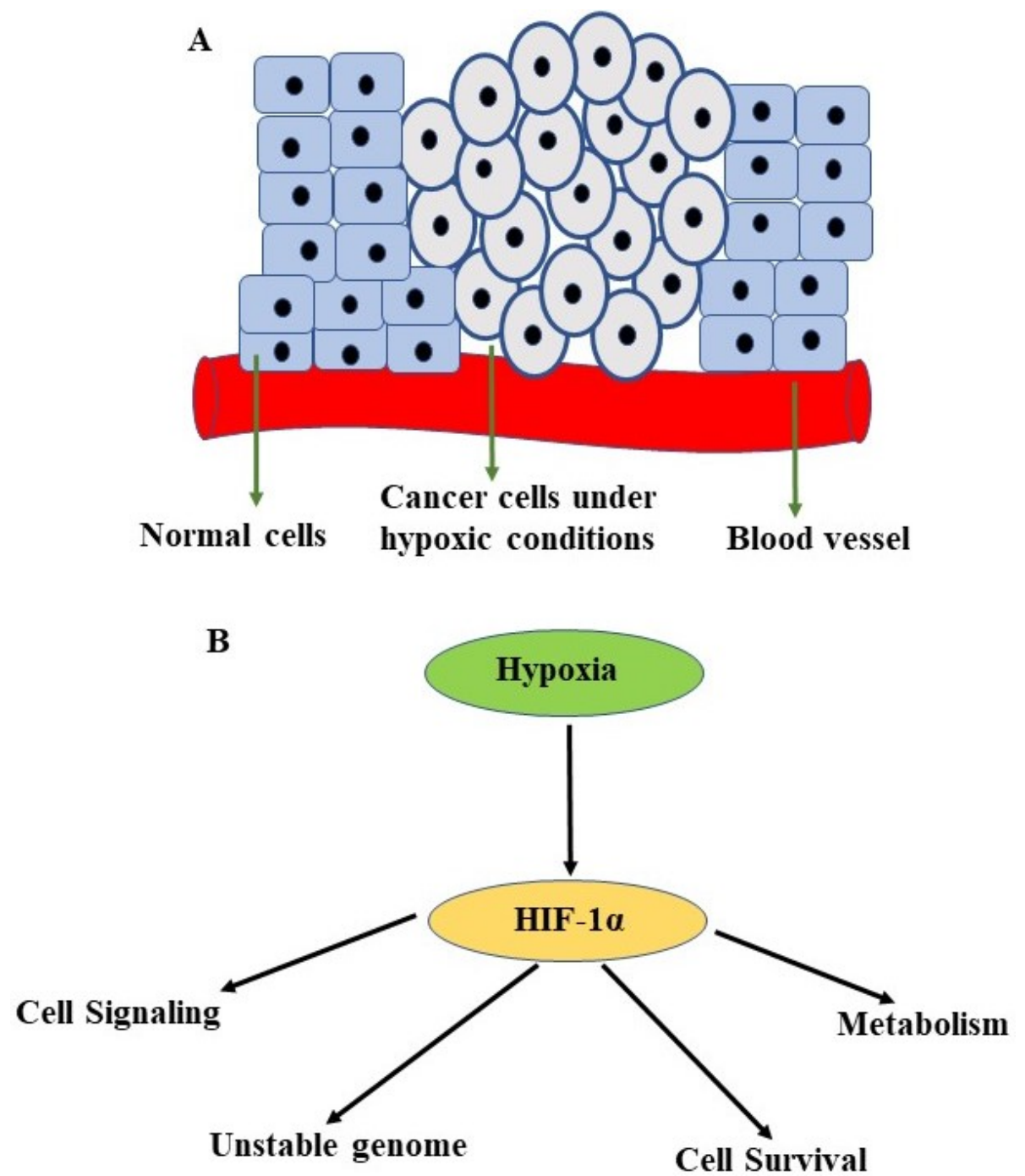

Figure 1-10. Various Functions of Hypoxia

(A) Schematic showing the changes in morphology of normal and cancer cells during hypoxia. (B) The involvement of hypoxia in various cellular functions is mentioned. 
mTOR complex subsist in two different isoforms viz., mTORC1 and mTORC2. The phosphorylation site for mTORC1 is S2448, whereas for mTORC2, it is S2481. Also, mTORC1 is sensitive to rapamycin, but mTORC2 is not. The two complexes are different in the type of proteins they are associated with [72]. The mTORC1 is comprised of mTOR, mLST8 and raptor proteins while mTORC2 complex is composed of mTOR, PRR5, mSIN1, mLST8 and rictor proteins [73].

The major proteins involved in mTORC1 pathway are 4EBP1 and S6kinase. On the other hand, AKT is the main player in mTORC2 pathway. Inefficient functioning of this pathway leads to various pathological conditions such as: diabetes, cancer, and heart diseases [73].

\section{Apoptosis}

This is one of the most common mechanisms involved in multicellular organisms. It is also known as programmed cell death. This mechanism is important for cellular homeostasis and proper development of the organisms [74]. Apoptosis is subdivided into extrinsic, and intrinsic pathways [75]. These two pathways (both intrinsic and extrinsic) have distinct functions in promoting cell death, that enables conventional functioning of the cellular processes.

During the extrinsic process, many proteins are involved that activate apoptosis. These proteins are specifically associated with the death receptors, such as Fas, the tumor necrosis factor (TNF) and TRAIL (TNF-related apoptosis-inducing ligand) [76, 77]. This process activates another protein, Fas-associated death domain (FADD), leading to the creation of DISC (death-inducing signaling complex). This is followed by stimulation of procaspase 8 and other caspases, finally resulting in apoptosis of the cells [74].

The intrinsic pathway occurs in the mitochondria, where it begins with the lysosome breakdown followed by several steps. Upon activation, the proteins associated with the Bcl-2 family produces cytochrome c after mitochondrial translocation. Thereafter, caspase- 3 is activated, and apoptosis occurs through key associated proteins [78].

\section{Translation Initiation}

The translation initiation process in eukaryotes is very complex. This phenomenon involves many factors and proteins, each operating different functions. Mainly, there are 6 eukaryotic initiation factors (eIFs): eIF1, eIF2, eIF3, eIF4, eIF5 and eIF6 [79]. It has been suggested that inappropriate regulation of the translation machinery results in tumor initiation and progression. Therefore, the molecules associated with this machinery are considered as emerging targets for therapeutic intervention of cancer [79]. The main proteins involved with the translation initiation complex are: eIF4B, eIF4E and eIF4G. They form a complex and tightly regulate the translation process. 
In the normal cells, this process is involved in the expression of genes, energy sources, and formation of ribosome. Whereas, in the tumorigenic status, the translation initiation pathway translates to a highly oncogenic form [80, 81].

\section{Functional Role of MUC13}

MUC13, a mucin member of glycoprotein family with molecular weight of 50$150 \mathrm{kDa}$ with induced expression pattern in pancreatic cancer. It has minimal expression in normal cells and has been reported to play a significant role in cancer progression, as well as modulating several tumorigenic signaling pathways [82].

In our previous publication, MUC13 has been reported to interact with Her2 protein, which promotes the progression of PDAC [83]. This protein also has enormous clinical importance as a diagnostic biomarker for pancreatic cancer, as elucidated in our recent publication [84]. However, the role of MUC13 in driving aberrant glucose metabolism in pancreatic cancer has never been investigated.

\section{Structure of MUC13}

The MUC13 structure is mainly composed of two domains: $\alpha$-domain and $\beta$ domain. The $\alpha$-domain consists of the TR (Tandem Repeat) domain, and the EGF-like 1 domain. In addition, the $\beta$-domain has the EGF-like 2 and 3 regions, TM (transmembrane domain), and the cytoplasmic-tail domain (containing the phosphorylation sites). The intermediate domain (SEA) is the cleavage region for proteins, and also contains a motif region for interaction between proteins.[82]. These domains of MUC13 protein are responsible for the oncogenic profile.

The large domain with glycosylation sites outside the cells primarily contributes for attachment of the cells with outside matrix. On the other hand, the cytoplasmic domain of MUC13 is involved in the cell signaling mechanisms, as it contains the phosphorylation sites. This protein is suggested to have potential as a biomarker to detect cancer [85]. The structure of MUC13 is shown in Figure 1-11.

\section{Functional Aspects of PKD1}

PKD1 is a kinase protein which plays important roles in many signaling pathways. It is involved in cell proliferation, cell survival, cell-cell adhesion, cell migration, etc. There are three family members of the Protein Kinase D family: PKD1/2/3 [86]. Among the three isoforms, PKD1 is the most prevalent member of the family with an extensive functional role in progression of pancreatic cancer. It is mainly present in the cytoplasm of cells, and in sparse amount in the Golgi body and also in the mitochondria [86]. 


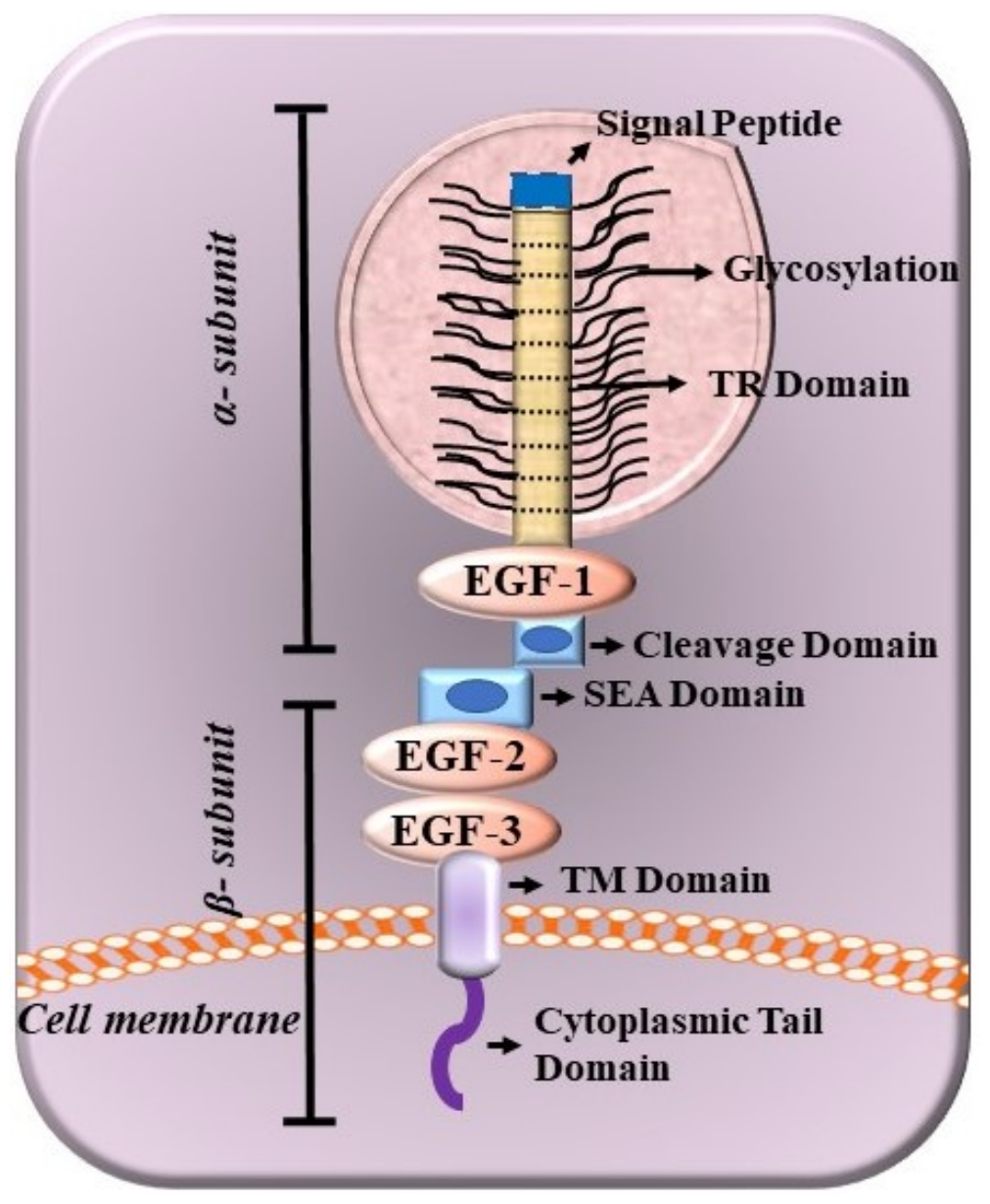

Figure 1-11. Structure of MUC13 Protein

Representative figure depicting the structural components of MUC13. The different domains of this protein are involved in various functions. SEA domain contains the cleavage site, and lies between the $\alpha$-subunit and the $\beta$-subunit of the MUC13 protein. The cytoplasmic domain comprises of a Protein Kinase C (PKC) motif, which suggests its role in various cell signaling processes. TR and TM domain refers to tandem repeat and transmembrane domains respectively. (Idea of this figure was taken from Maher $e t$ al., 2011 [85]). 
Previous reports have indicated that PKD1 can be a marker for tumor detection in the initial stages, and also be a promising drug target for pancreatic cancer [87] Another study suggested that stimulation of PKD1 through oxidative stress can lead to pancreatic tumor invasive phenotype [88] Other researchers had previously reported that PKD1 enhances growth, angiogenesis, and invasive features of pancreatic cancer, and thus can be exploited for therapeutic purposes [89]. Despite of having a potential role in pancreatic cancer pathogenesis, the underlying signaling mechanisms regulated by PKD1 has not been completely elucidated. So far, no studies have been investigated about the clinical implications of PKD1 in glucose metabolism of pancreatic cancer.

\section{Structure of PKD1}

PKD1 protein is composed of 912 amino acids with molecular weight of $115 \mathrm{kDa}$. The structure of PKD1 is divided into two distinct domains: the regulatory domain and the catalytic domain flanked through the $\mathrm{N}$-terminal to the $\mathrm{C}$-terminal regions [86].

The regulatory region is composed of alanine-proline (AP) rich domain, cysteinerich domain, amino-acid domain and a pleckstrin homology $(\mathrm{PH})$ domain [86]. Each of these regions possess distinct functions. The exact function of the AP region has not been elucidated yet. However, its suggested possible role can be in lipid binding [90], and providing proper protein conformation as well as maintenance of PKD1 stability [91]. The cysteine-rich regions are essential for binding of lipids, and the $\mathrm{PH}$ region is involved in interaction of protein with proteins/lipids [92]. The structure of PKD1 is shown in Figure 1-12.

In this dissertation, we demonstrate the role of MUC13 and PKD1 involved in regulating glucose metabolism, further leading to growth and aggressive metastatic phenotypes of pancreatic cancer (Chapter 2 and 3).

\section{Current Treatment Options for Pancreatic Cancer}

Very limited number of treatment options are available for pancreatic cancer. If detected early, surgery is also an option. But in the metastatic state, a combination of drugs named, FOLFIRINOX is employed. This chemotherapy option contains the drugs: oxiplatin, leucovorin, fluorouracil and irinotecan [93]. The available therapeutic options for pancreatic cancer are: chemotherapy, radiotherapy, resectable surgery and combination of chemotherapy and radiotherapy [94] Most of the times, the standard regimen employed is the drug gemcitabine along with 5-FU (5-fluorouracil) [95]. Gemcitabine is preferred over other drugs as it has minimal toxicity and high tolerance capabilities [96]. On the other hand, 5-FU is mainly used when a patient is unable to respond to gemcitabine [97].

It has been observed that all patients do not have uniform response towards

FOLFIRINOX. Therefore, other combinatorial options have been approved by FDA such 


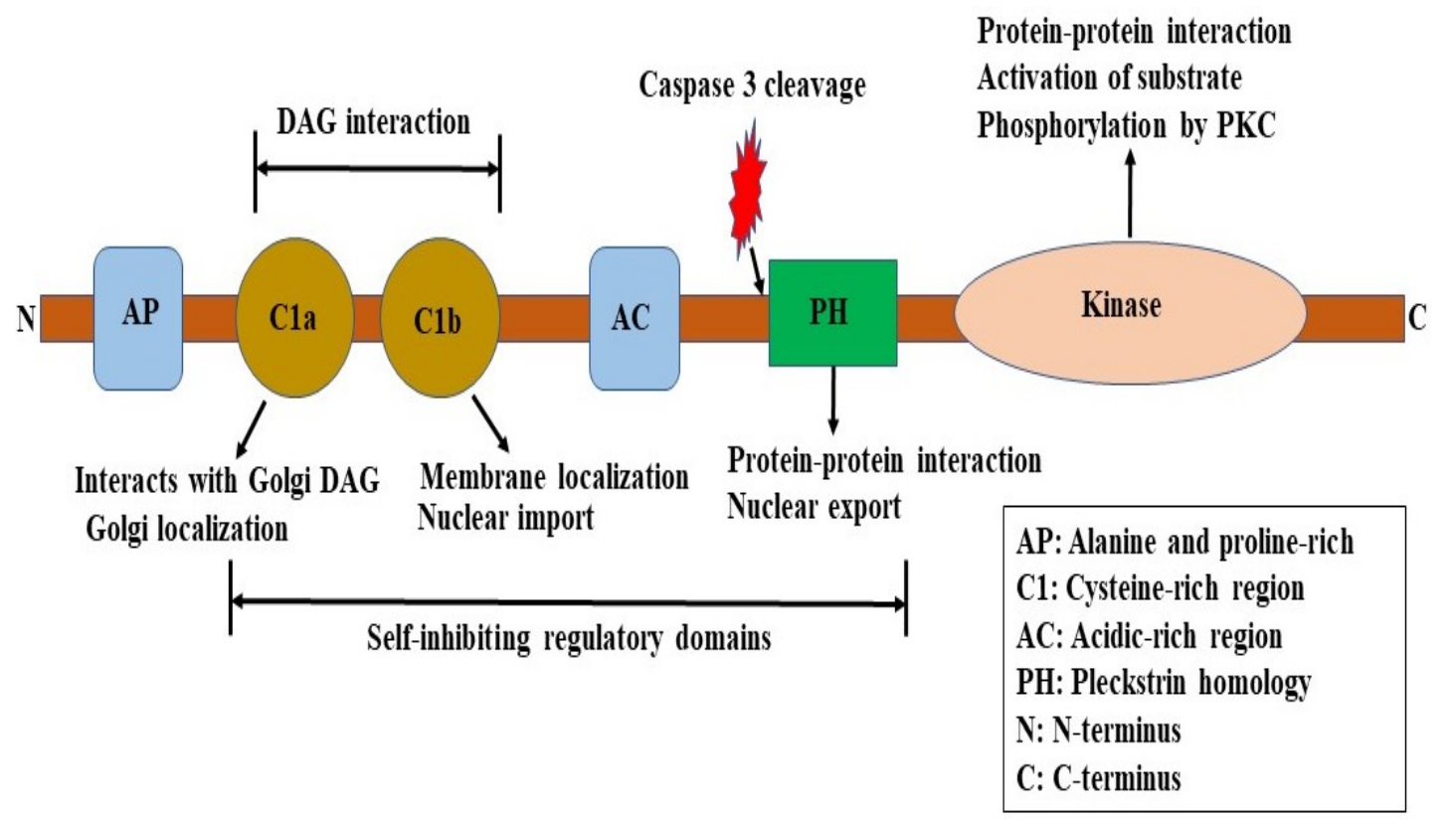

Figure 1-12. Structure of Protein Kinase D1

Schematic diagram representing the different functional domains of PKD1 protein. The various domains of PKD1 protein are mentioned in the inset. The activation/phosphorylation of the kinase domain occurs at S744 and S748 sites. This in turn leads to release of inhibition by itself and also at S916 site, autophosphorylation occurs. (The idea of this schematic diagram emerged from Sundram et al., 2011 [86]). 
as paclitaxel (albumin-bound) along with gemcitabine, and they have demonstrated better efficiency [98]. Accumulating evidences suggest that aberrant glucose metabolism plays pivotal role in the development, progression and metastasis of pancreatic cancer. Several glucose metabolic targeting agents have been discovered which show potent therapeutic efficacy against pancreatic cancer and are discussed below.

\section{Molecular Targeting of Glucose Metabolism in Pancreatic Tumors}

Therapies targeting tumor metabolism can inhibit growth of tumors. The tumor metabolism can be inhibited by reduction of glucose uptake and its further secretion into lactate. It can also be targeted by inhibition of signaling pathways involved in cancer growth and less availability of essential nutrients required for growth and proliferation.

Previous research had suggested that 2-deoxyglucose (2-DG) treatment, which is a known suppressor of glycolysis, results in reduction in pancreatic cancer cell viability mediated by oxidative stress mechanism and also by abnormalities in thiol metabolic pathway [99]. Recent studies by Chapiro et al. [100], demonstrated the therapeutic efficacy of microencapsulated 3-bromopyruvate (3-BrPA), which was assessed in orthotopic xenograft mouse models of PDAC. It represented the systemic delivery of the formulation as an antiglycolytic tumor treatment. The results showed remarkable anticancer effects with no toxicity, which warrants clinical trials in PDAC patients [101]. One of the mTOR inhibitors, everolimus is also a metabolism inhibitor, and has shown promising results in pancreatic tumors, as well as several other types of tumors [102]. EZN-2968 is an antisense oligodeoxynucleotide and an inhibitor of HIF-1 $\alpha$, which has demonstrated distinct results in phase I clinical trials [103].

Previous studies have suggested that the various sources of energy are responsible for the different metabolic phenotypes in PDAC. Therefore, targeting these phenotypes with their individual inhibitors can provide an effective therapeutic strategy for PDAC patients [104]. The derivative of perylene (TEL03) is known to regulate HIF-1 $\alpha$ and STAT3 signaling. This derivative is a dual inhibitor, which can target both these pathways, in pancreatic cancer [105]. Previous research had shown that the histone deacetylase (HDAC) inhibitor, chidamide is a potent anti-cancer agent. The researchers have identified that its mechanism of action is by inhibiting the aerobic metabolism and degradation of Mcl-1, and leading to reduced cell proliferation of pancreatic cancer cells [106]. Recent studies have identified the anti-cancer role of this inhibitor in pancreatic cancer cells [107]. Chidamide is being tested in clinical trials in China and the US for its therapeutic efficacy in pancreatic cancer [108]. A TGF-beta receptor I kinase inhibitor, galunisertib, has shown promising results in phase I clinical trials in pancreatic cancer patients [109].

The glycolysis of tumor cells can be exploited as attractive molecular target in chemotherapy. [110]. Some researchers have highlighted the importance of mitochondrial glutaminase 1(GLS1) inhibitors and $\beta$-lapachone for high selectivity in PDAC patients [111]. New researches involve the combined use of modulation of the autophagy 
mechanism (through autophagy inhibitors) with anticancer therapy regimens [112]. Another inhibitor which is undergoing investigation is metformin. It is an inhibitor of the electron transport chain [113]. Other research groups have suggested the importance of metformin and some $\beta$-blockers for controlling the emergence of pancreatic cancer [114]. In a recent research, an inhibitor of glutamine was used and through nanoparticle-based drug delivery approach, in combination with metformin, reduced pancreatic tumor growth [115].

Hexokinase II (HKII) facilitates the conversion of glucose to Glu-6-P (glucose 6phosphate) by its enzymatic action in glycolysis. An inhibitor of HKII, lonidamine had undergone phase III clinical trials, but was unsuccessful due to some toxicities [116]. 2Deoxyglucose (2-DG) is an analog of glucose, which showed anti-cancer effects, but also increased pro-survival activities [117]. Also, it was reported that during hypoxia, the cells became chemo-resistant to this drug [118]. Although this drug has not been successful alone, but in combination has shown promising results in other types of cancer [119, $120]$.

Two of the most studied drug candidates for cancer prevention are aspirin and metformin in the recent years. Both these drugs decrease cell proliferation in pancreatic cancer cells [121]. Some researchers have conducted transcriptomic studies in order to analyze the gene regulation of these drugs [122]. Also, investigations have been performed for metabolomic profiling using serum samples from pancreatic cancer patients and control group. This included mass spectrometry studies to obtain knowledge about some biomarkers for diagnostic purpose [123]. Moreover, several inhibitors have been identified in recent years for targeting lactate metabolism. FX-11 is an inhibitor of lactate dehydrogenase A (LDHA), which has been reported to retard the proliferation of pancreatic cancer cell lines [124]. Also, several potential mitochondrial activity inhibitors have been tested for their efficacy, such as: berberine, resveratrol, curcumin and epigallocatechin gallate [125].

We have evaluated the efficacy of a phytochemical drug for targeting dysregulated metabolism in pancreatic cancer in this research work, which is described below.

\section{Steviol}

Steviol is plant product from Stevia rebaudiana, with a molecular weight of $318.45 \mathrm{kDa}$. It is a natural sweetener, which is almost 300 times sweeter than cane sugar. Several studies have indicated its beneficial properties in being anti-inflammatory, and anti-hyperglycemic. Reports also suggest its anti-cancer properties in gastrointestinal cancers and breast cancer [126-129]. However, its role in pancreatic cancer still remains unidentified.

Steviol is formed through conversion of steviol 16, $17 \alpha$ epoxide from its precursor, stevioside, through the bacteria in the human gut [130]. Reports have indicated 
that the precursor molecule, stevioside is very efficient in lowering the blood glucose levels in humans suffering from diabetes [131], and similar observations were also found in rats [132]. These reports indicate the efficacy and benefits of this drug for effective glucose metabolism. The chemical structure of steviol is depicted in Figure 1-13.

In this dissertation, we have evaluated the therapeutic efficacy of this natural product (Steviol), which suppresses the growth and metastatic phenotypes of pancreatic cancer (Chapter 4).

\section{Rationale of the Study}

Altered glucose metabolic phenotype is a hallmark of pancreatic cancer [133]. It is essential to identify molecular targets and drugs which can suppress elevated glucose metabolism. MUC13 protein is a transmembrane glycoprotein (mucin) with high expression levels in pancreatic cancer. This protein offers fueled tumor microenvironment to growing cells for adoption of their enhanced tumorigenic behavior $[82,85]$. Some of the mucins such as MUC1 $[134,135]$ and MUC16 [136] have been reported to play a significant role in abnormal glucose metabolism. However, the role of MUC13 in upregulated tumor metabolism had never been investigated earlier. Therefore, we were interested in deciphering this aspect of MUC13. Further, in order to find some other molecular targets involved in abnormal cancer metabolism, we found a kinase protein, Protein Kinase D which had been reported to have an important role in cardiac glucose uptake leading to hypertrophy [137]. Also, Protein Kinase D1 (PKD1) has been recognized to have significant role in hypoxic tumor metabolism [138]. Nevertheless, its involvement in cancer glucose metabolism was never studied. So, it was interesting to investigate this molecule and the associated signaling mechanisms pertaining to tumor metabolism. Also, it was mandatory to identify natural agents which could restore glucose metabolism in pancreatic cancer, with no to minimal side effects. For this, several compounds were screened, which finally led to the identification of an important constituent, steviol from the plant Stevia rebaudiana, that could inhibit enhanced glucose consumption and associated altered metabolism in abnormal cells.

\section{Hypothesis of the Overall Study}

Dysregulation of glucose metabolism plays a crucial role in the initiation, progression and metastatic status in pancreatic cancer. The regulatory factors (MUC13/PKD1) responsible for deviant glucose metabolism need further investigation in order to develop effective therapies. Also, we hypothesize that steviol can suppress the altered glucose metabolism, ultimately leading to less invasive pancreatic cancer. 


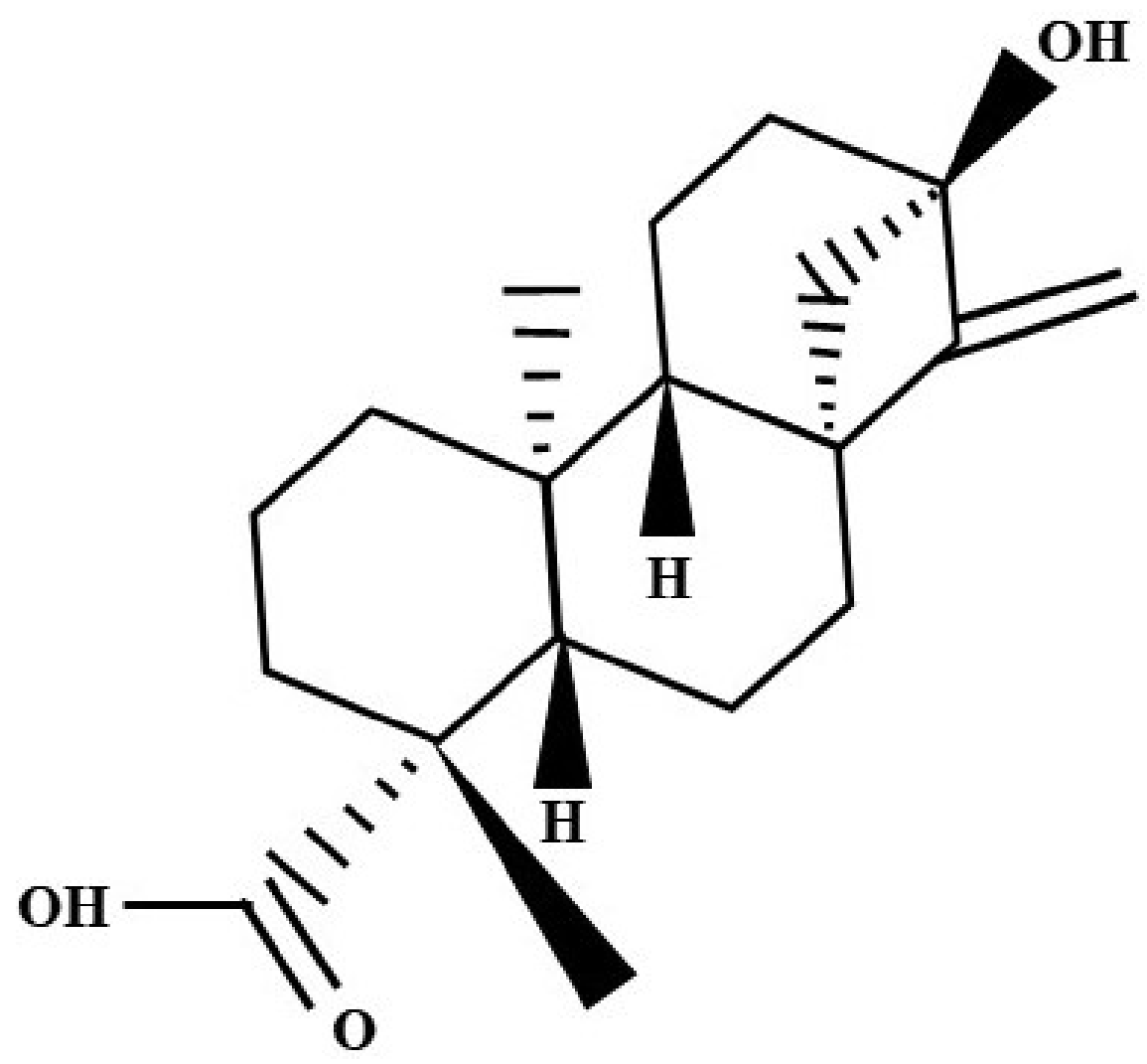

Figure 1-13. Chemical Structure of Steviol 


\section{Objective of This Project}

To identify the oncogenic proteins involved in metabolic reprogramming of pancreatic cancer, the underlying mechanisms involved, as well as examining the potential role of non-toxic natural agents in reducing the elevated glucose metabolism and its impact on pancreatic cancer.

\section{Specific Aims of the Project}

Aim 1: To determine the role of a mucin, MUC13 in glucose metabolism and to investigate the molecular interactions of MUC13 with the key transporter of glucose, Glut-1 in pancreatic cancer.

Aim 2: To investigate role of Protein Kinase D1 in glucose metabolism of pancreatic cancer and the underlying mechanisms.

Aim 3: To investigate the role of a natural agent (Steviol) in glucose metabolism of pancreatic cancer, and study the mechanistic pathways involved. 


\section{CHAPTER 2. MUC13 CONTRIBUTES TO REWIRING OF GLUCOSE METABOLISM IN PANCREATIC CANCER ${ }^{1}$}

\section{Introduction}

Pancreatic cancer will be the second leading cause of cancer-related deaths very soon as per future projections [139]. Due to the late diagnosis, chemo-resistance and metastasis, 5-year survival rate still remains less than $8 \%$ [139]. The pancreas has an anatomically inaccessible location that prevents routine examination, early stage diagnosis and timely surgical resection of tumor(s). Additionally, because of highly aggressive nature, pancreatic cancer cells disseminate rapidly leading to early metastasis of the disease. At this stage, available treatment options are only moderately successful to extend the patient's survival rate. Therefore, it is highly important to understand and delineate the molecular pathways that lead to increased tumor aggressiveness and poor patient survival rate. One such unique characteristic physiology of pancreatic tumors includes a hostile and tumor microenvironment, which favors biochemical and metabolic adaptations to facilitate pancreatic tumor growth and metastasis. Alterations in metabolic pathways, which have a common feature of increased glucose uptake and its conversion to lactate, are highly responsible for cancer progression [140].

Cancer cells undergo an oncogene addiction, which makes them highly dependent on the activity of an oncogene for survival and proliferation [141]. The fibrotic pancreatic tumor microenvironment enables pancreatic cancer cells to rely on alternative sources of nutrients and adapt distinctive approaches to obtain them. Most cancer cells exhibit enhanced aerobic glycolysis, known as the Warburg effect, an alteration in glucose metabolism resulting in an acidic tumor microenvironment that facilitates local invasion of tumor cells [142]. Warburg effect is the enhanced conversion of glucose to lactate observed in tumor cells, even in the presence of normal levels of oxygen. Glucose is the major metabolic precursor that supports the Warburg effect and glucose transporter 1 (Glut-1) mediates cellular glucose transport required to fuel anaerobic metabolism in proliferating cancer cells [143]. The increased lactate production results in enhanced tumorigenic characteristics such as cell invasion, migration, metastasis of the tumor and correlates with tumor reoccurrence [144]. Thus, oncogenic networks required for this process, are highly desirable to develop novel strategies for selective targeting of aberrant pancreatic tumor metabolism and tumor microenvironment. Pancreatic cancer metabolism is dramatically rewired by oncogenic KRAS that induces a series of metabolic alterations, which includes enhanced glycolysis and glutaminolysis, resulting in enhanced cell growth and proliferation [145]. Hypoxia is one of the factors in the pancreatic tumors that leads to enhanced glucose metabolism, hypoxia-inducible factor 1

${ }^{1}$ Reprinted from final submission with open access permission. Kumari S, Khan S, Gupta SC, Kashyap VK, Yallapu MM, Chauhan SC, Jaggi M. MUC13 contributes to rewiring of glucose metabolism in pancreatic cancer. Oncogenesis. 2018 Feb 22; 7(2):19. doi: 10.1038/s41389-018-0031-0 [11]. 
(HIF-1) being an important regulator of cellular oxygen homeostasis [146]. NFkB plays an important role to regulate the metabolic adaptation in normal and cancer cells by controlling the balance between the utilization of glycolysis and mitochondrial respiration [147].

Mucins are a family of high molecular weight and glycosylated proteins that play significant roles in pancreatic cancer pathogenesis [148]. MUC13 protein, is a transmembrane mucin, which is aberrantly expressed in pancreatic cancer [82] and enhances pancreatic tumor progression through various mechanisms; one such mechanism is being mediated through its interaction with HER2, a member of EGFR family [83]. In the present study, we report a novel role of MUC13 in metabolic reprogramming of pancreatic cancer. We demonstrate the metabolic alterations induced by MUC13 in pancreatic cancer cells and the underlying molecular mechanisms that drive the associated tumorigenic characteristics. Our studies indicate that the MUC13induced metabolic alterations require $\mathrm{NF} \kappa \mathrm{B}$ activation that precedes the triggering of events in controlling glucose metabolism in pancreatic cancer cells. In addition, we demonstrated the functional interaction of MUC13 and Glut-1 and established their positive correlation among patient clinical samples belonging to pancreatic ductal adenocarcinoma. Briefly, our study delineate role of MUC13 in aberrant glucose metabolism in pancreatic cancer and suggest it as a novel molecular target for therapeutic intervention of this frightening disease.

\section{Materials and Methods}

\section{Cell Culture}

MUC13 null pancreatic cancer cells, Panc-1 and MiaPaca (P-V, M-V), and stably expressing MUC13 (P-M13, M-M13) were used for the study, as described earlier [82]. We also generated MUC13 expressing HPAF-II (sh-V) and knockdown (sh-M13) cells using a lentiviral transfection, for our study. Cells were maintained in DMEM or DMEM/F12 media supplemented with $10 \%$ fetal bovine serum and $100 \mu \mathrm{g} / \mathrm{ml}$ of G418 antibiotic or $5 \mu \mathrm{g} / \mathrm{ml}$ puromycin. These cells were grown at $37^{\circ} \mathrm{C}$ in an incubator which supplied 5\% CO2 in a humidified environment.

\section{Clinical Samples}

Human pancreatic cancer tissue samples were procured from Baptist Memorial Health Center. Additionally, cDNA of pancreatic cancer tissue specimens was purchased from Origene. 


\section{Immunoblotting}

Whole cell lysates were prepared followed by immunoblotting as described earlier $[83,149,150]$. The expression level of several proteins were analyzed using specific primary antibodies obtained from Cell Signaling: NFкB p-65 (catalog number: 8242), phospho-NFкB p-65, Ser536 (catalog number: 3033), ІкB $\alpha$ (catalog number: 4814), phospho- IкB $\alpha$, Ser32/36 (catalog number: 8219), Histone H3 (catalog number: 4499), GAPDH (catalog number: 5174), HIF-1 $\alpha$ (catalog number: 3716), Bcl-2 (catalog number: 4223), c-Myc (catalog number: 9402), Glut-1 (catalog number: 12939), alphatubulin (catalog number: 2144), p27 Kip1 (catalog number: 2552), Ras (catalog number: 3965), KRAS-12D (catalog number:14429). The anti-MUC13 monoclonal antibody used for this manuscript was produced in our lab. The secondary antibodies for rabbit (catalog number: 4011), and mouse (catalog number: 4021) conjugated with horseradish peroxidase were obtained from Promega.

\section{Reciprocal Co-Immunoprecipitation}

The reciprocal co-immunoprecipitation experiments were performed using AsPC1 and HPAF-II cells, which express MUC13. 500 $\mu \mathrm{g}$ of protein was used and incubated with antibodies at $4^{\circ} \mathrm{C}$ overnight. Immuno-precipitates were eluted with sodium dodecyl sulfate sample buffer obtained from Santa Cruz Biotechnology, TX, USA following the procedures as described earlier [83, 151].

\section{Confocal Immunofluorescence}

Confocal immunofluorescence was performed as described earlier [83, 150]. Briefly, cells were fixed and incubated with respective primary antibodies overnight. This was followed by incubation with $\mathrm{Cy} 3$, or Alexa Fluor 488, donkey secondary antibodies for 1 hour. The images were then captured at 400X magnification using a confocal microscope (Nikon Corporation, Melville, NY, USA).

\section{Wound Healing/Scratch Assay}

Cell migration assay was performed using wound healing assay [149, 152]. Cells were seeded in 12 well plate at a density of $2 \times 105$ cells. The cells were treated with LLactate $(2 \mathrm{mM})$ and 2-Deoxyglucose $(10 \mathrm{mM})$ and further incubated for 24 hours. The cells were photographed at 0 and $24 \mathrm{~h}$. 


\section{Invasion Assay}

Cell invasion assay was performed using BD Biocoat Matrigel Invasion Chambers (BD Biosciences), as described earlier [149]. Cells were seeded in matrigel invasion chambers in a serum free medium. The cells were treated with L-Lactate $(2$ $\mathrm{mM})$ and 2-Deoxyglucose $(10 \mathrm{mM})$ followed by incubation for 24 hours. Then, the cells were fixed using methanol and were stained with crystal violet. The images were captured at 24 hours.

\section{Cell Proliferation Assays}

Cell proliferation assay was performed in MUC13 null (P-V) and MUC13 expressing (P-M13) cells after treatment with lactate $(2 \mathrm{mM})$ and 2-DG $(10 \mathrm{mM})$. The experiment was terminated after $48 \mathrm{hrs}$ with the addition of $20 \mu \mathrm{l}$ of tetrazolium dye, MTT reagent: 3-(4,5-dimethylthiazol-2-yl)-2,5-diphenyltetrazol-2-yl)-2,5-diphenyltetrazolium bromide. After $2 \mathrm{hrs}, 100 \mu \mathrm{l}$ of dimethyl sulfoxide was added to the cells and was kept on shaker for 10 minutes. Finally, the absorbance was recorded at 570 $\mathrm{nm}$, and the percentage cell viability was quantified.

\section{Lactate and Glucose Assays}

Lactate and Glucose assays were performed using kits from Cayman Chemicals (Lactate assay kit catalog number: 600450, Glucose assay kit catalog number: 10009582). MUC13 expressing and null cells were seeded (104 cells/ well in 96 wells plate) and media collected to measure the amount of lactate after 24 hours, and unused glucose levels after 48 hours. The samples were analyzed according to the instructions provided in the kit, the readings were recorded and calculations were done.

\section{Isolation of RNA and Polymerase Chain Reaction (PCR)}

RNA from pancreatic cancer cells and tissues was isolated using Qiagen kit and quantified using Nanodrop instrument 2000 (Thermo Scientific). RNA $(2 \mu \mathrm{g})$ was reverse transcribed using SYBR green RNA Reverse Transcription kit. The expression of genes was determined by using specific primers by qPCR and semi quantitative PCR. GAPDH was used as an internal control for the reactions.

\section{In Situ Proximity Ligation Assay (PLA)}

This assay was performed using the Duolink Red Starter PLA Kit as described earlier [83] to detect the subcellular localization of the protein-protein interaction at a single-molecule resolution. Briefly, oligonucleotide-conjugated anti-mouse minus and anti-rabbit plus PLA secondary probes were added and incubated in a humidified 
chamber for 1 hour at $37{ }^{\circ} \mathrm{C}$. The oligonucleotides were ligated for 30 minutes at $37{ }^{\circ} \mathrm{C}$ to produce rolling circle amplification products tagged with a red fluorescence probe.

Further, the nuclei were counterstained with 4', 6-diamidino-2-phenylindole (DAPI), and were then visualized using a Zeiss confocal microscope.

\section{Co-Capping Assay}

This assay was performed using MUC13-expressing HPAF-II and AsPC1 cells as described before [83]. The cells were incubated with NHS-Rhodamine-labeled antiMUC13 mAb $(30 \mu \mathrm{g} / \mathrm{ml})$ for 1 hour at $4{ }^{\circ} \mathrm{C}$, and plated for adherence at $37{ }^{\circ} \mathrm{C}$ for about 2 hours. The cells were incubated with Glut-1 (Cell Signaling, catalog number: 12939), or $\alpha$-tubulin negative control (Cell Signaling, catalog number: 2144), overnight. Cells were further incubated with Alexa-Fluor 488 anti-Rabbit secondary antibody, and the images captured using 710 Zeiss confocal microscope [150].

\section{Statistical Analysis}

The data in this manuscript were evaluated and analyzed using Microsoft excel software, and the statistical analysis is represented as means+/-SEM of three independent experiments. In all cases, the significance of the data was analyzed through unpaired twosided Student's $t$ test, and p-value less than 0.05 was considered statistically significant.

\section{Results}

\section{MUC13 Enhances Glucose Metabolism and Invasiveness in PDAC Cells}

Processes in malignant transformation involve enhanced glucose uptake and lactate production which consequently lead to the defects in the expression of glycolytic enzymes and metabolite transporters and in oncogenic alterations [153]. In order to investigate the role of MUC13 on glycolytic properties of pancreatic cancer cells, glucose and lactate assays were performed in MUC13 null Panc-1/MiaPaca cells (P-V/M-V) or stably MUC13 overexpressing (P-M13/M-M13) cells. We observed significantly $(\mathrm{p}<0.05)$ higher upregulation of L-lactate production and glucose consumption in MUC13 expressing cells as compared to MUC13 null control cells (Figure 2-1A,.B). Therefore, our results demonstrate that MUC13 enhances glucose metabolism of pancreatic cancer cells. Previous reports demonstrate that MUC13 enhances invasion and migration in pancreatic cancer cells. In this study, our observations suggest that MUC13 expression modulates glucose consumption in PDAC cells, therefore, we sought to investigate if MUC13 induced changes in proliferation, invasion and migration are mediated through altered glucose metabolism. We demonstrate that MUC13 expression (P-M13 and M-M13) cell invasion (Figure 2-1C, D) and migration (Figure 2-1E, F) that can be further enhanced by adding lactate $(2 \mathrm{mM})$, an end product of aerobic glycolysis, 

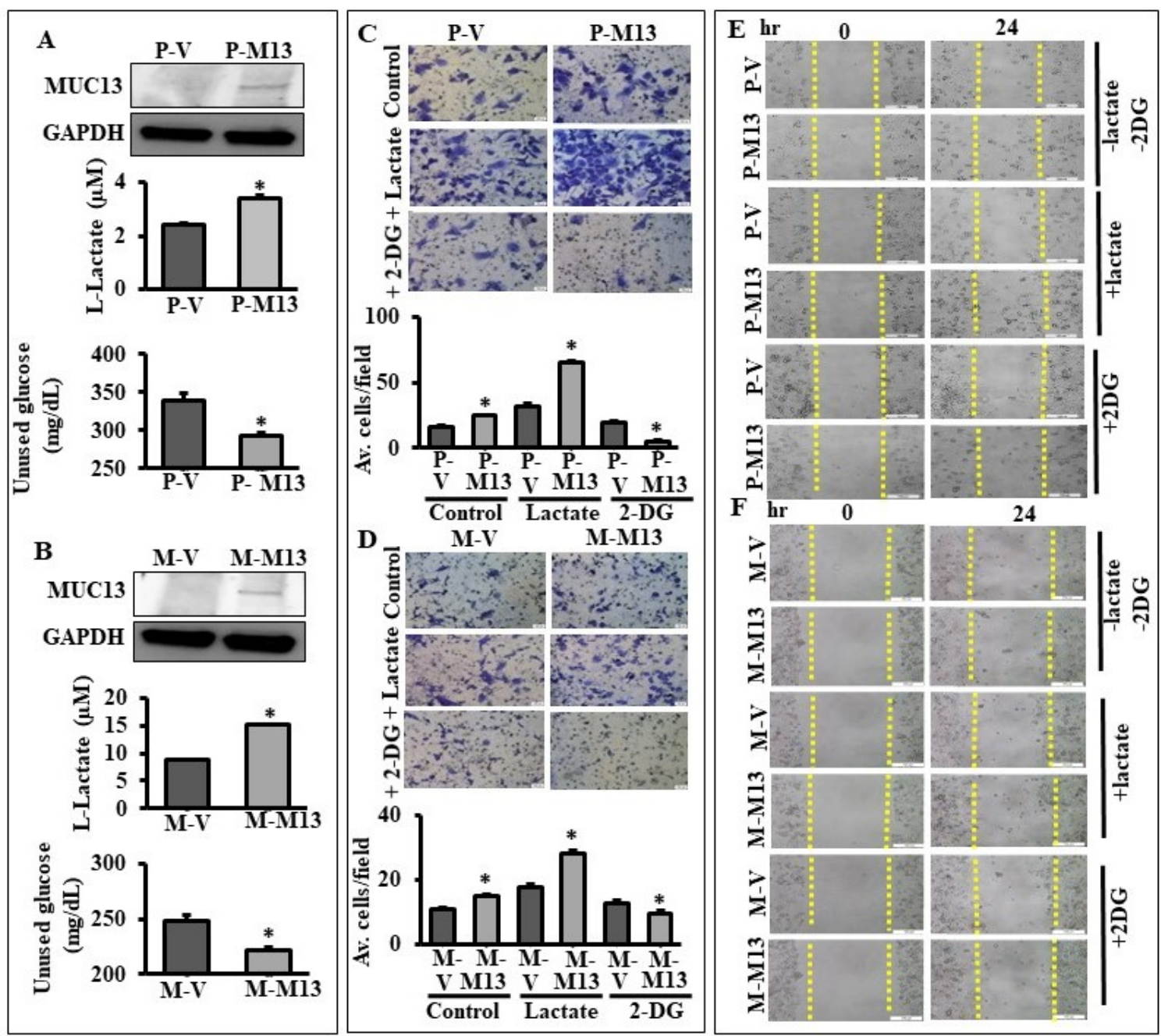

Figure 2-1. MUC13 Enhances Glucose Metabolism in PDAC Cells

Expression of MUC13 shown by Western blotting, lactate and glucose assay in MUC13 null Panc-1 (P-V) and stably expressing MUC13 (P-M13) cells (A) and in MUC13 null MiaPaca (M-V) and stably expressing MUC13 (M-M13) cells (B). Invasion assay performed in P-V and P-M13 (C) and M-V and M-13 cells (D) after treatment with lactate $(2 \mathrm{mM})$ and 2-DG $(10 \mathrm{mM})$. Images were captured after $24 \mathrm{hrs}$ and the relative number of invasive cells was quantified. Wound healing (scratch) assay performed in PV and P-M13 (E) and M-V and M-13 cells (F) after treatment with lactate ( $2 \mathrm{mM})$ and 2DG $(10 \mathrm{mM})$. The images were captured at 0 hrs and $24 \mathrm{hrs.} n=3 ;{ }^{*}<<0.05$. 
to the media in which cells are cultured. This effect, however, was abrogated by inducing glucoprivic conditions using 2-Deoxyglucose (2-DG, 10mM) (Figure 2-1C through F). These results suggest that MUC13 influenced metastatic characteristics of PDAC cells are partly the consequence of its modulation of the glucose metabolism.

\section{MUC13 Upregulates c-Myc Expression and the Downstream Effectors of Glucose Metabolism}

Since our results showed a marked upregulation of glucose uptake and lactate production, we evaluated effects of MUC13 expression on the important proteins involved in enhanced cancer cell metabolism. Immunoblotting and immunofluorescence analysis demonstrated an enhanced expression of c-Myc (Figure 2-2A, B). c-Myc is a master regulator of downstream processes that are involved in cell growth and metabolism [56]. The changes in c-Myc expression were accompanied by overexpression of glucose transporter1 (Glut-1), which ensures accumulation of glucose in the cell and secretion of lactate out of the cell (Figure 2-2A, B). Also, we observed that KRAS levels were induced in MUC13 expressing cells. This KRAS has prominence in regulating anabolic glucose metabolism [145]. One of the mediators of KRAS-induced transcriptional changes of metabolic genes is known to be HIF-1 $\alpha$, and its expression was found to be significantly higher upon MUC13 expression (Figure 2-2A, B) [145]. The mean fluorescence intensities for c-Myc, Glut-1 and HIF-1 $\alpha$ were also quantified using Zen software (Supplementary Figure S4 A and B). Additionally, MUC13 expressing cells were found to modulate proteins associated with cell death such as activation of antiapoptotic protein, Bcl-2 and inhibition of tumor suppressor/cell cycle inhibitor, p27 (Figure 2-2A). Immunoblotting and Immunofluorescence results were confirmed using qPCR analysis (Figure 2-2C) and semi-quantitative PCR (Figure 2-2D) showing increased mRNA expression of c-Myc, Glut-1 and HIF-1 $\alpha$ in MUC13 expressing Panc-1 and MiaPaca cells which was inhibited on MUC13 knockdown in HPAF-II cells (shMUC13). The activation of HIF-1 $\alpha$ prompted us to investigate whether MUC13 typically demands hypoxic environment to contribute to adaptive mechanisms of enhanced cell metabolism. Our results showed that the induced hypoxic conditions accompany enhanced production of lactate in MUC13 expressing cells as compared with MUC13 null cells (Figure 2-2E). The nuclear localization of c-Myc in MUC13 expressing Panc1 and MiaPaca cells (P-M13/M-M13) and the cellular localization of Glut-1 has been illustrated through confocal microscopy (Figure 2-3). Also, the localization of HIF-1 $\alpha$ protein in pancreatic cancer cells has been demonstrated by immunofluorescence techniques (Figure 2-4). These results indicate that MUC13 contributes to survival adaptation in hypoxic environment leading to enhanced metabolic changes for the survival of pancreatic cancer cells. This data suggest MUC13 expression induce rewiring of oncogenic signaling pathways that contribute to adaptive cellular mechanisms in tumor microenvironment. 

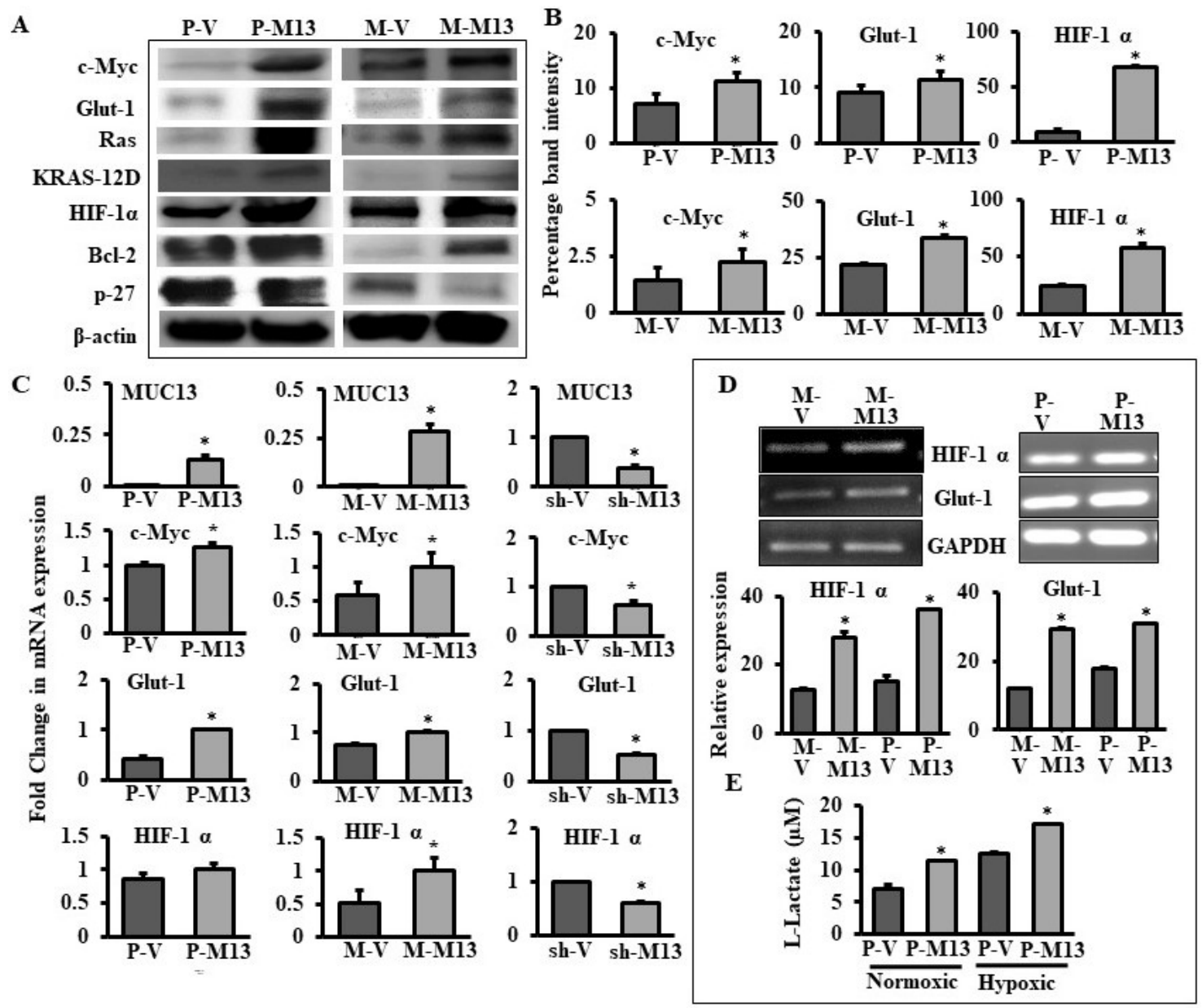

Figure 2-2. MUC13 Regulates Protein and mRNA Expression of Key Factors Involved in Glucose Metabolism

(A) Western blot showing the expression level of indicated proteins involved in glucose metabolism. Whole cell lysates were collected from cells and the levels of protein expression were determined. (B) Densitometric analysis of bands for c-Myc, Glut-1 and HIF-1 $\alpha . n=2 ; * p<0.05$. (C) qPCR depicting changes in mRNA expression in MUC13 null (P-V/M-V) cells and MUC13 expressing (P-M13/M-13) Panc-1 and MiaPaca cells; MUC13 expressing (sh-V) and knockdown (sh-M13) HPAF-II cells, ${ }^{*} p<0.05$. (D) Semiquantitative PCR performed to determine the expression of HIF-1 $\alpha$ and Glut-1 on MUC13 expression in PanCa cells and bars representing relative quantification of HIF-1 $\alpha$ and Glut-1 expression. (F) Lactate assay performed in Panc-1 cells under normoxic and hypoxic conditions. ${ }^{*} \mathrm{p}<0.05$. 
$\mathbf{A}$
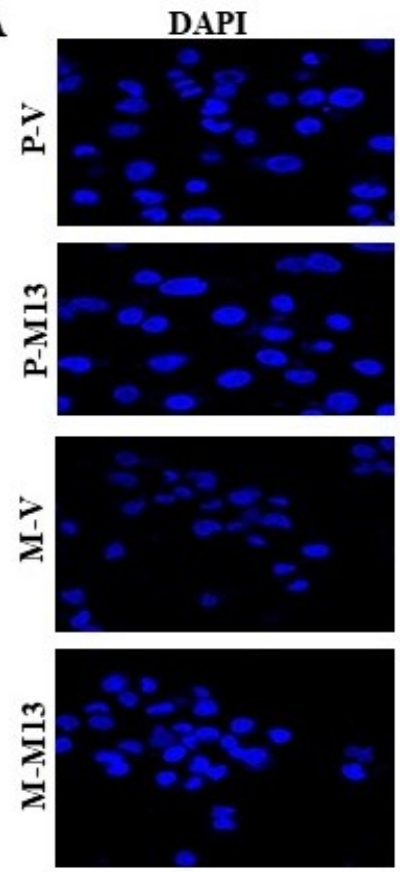

B
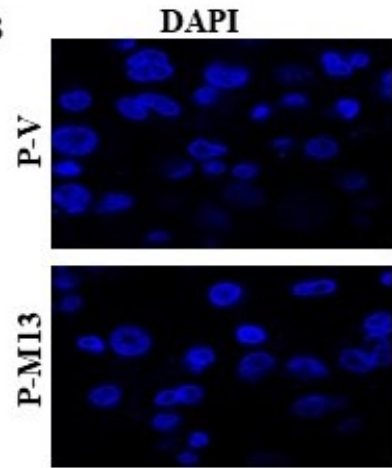

c-Myc
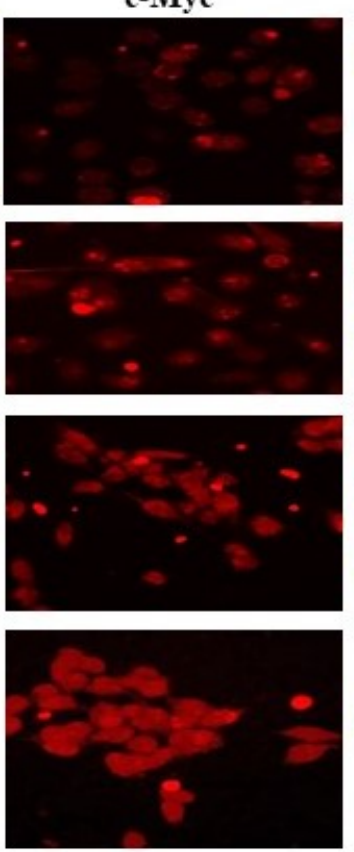

Glut-1
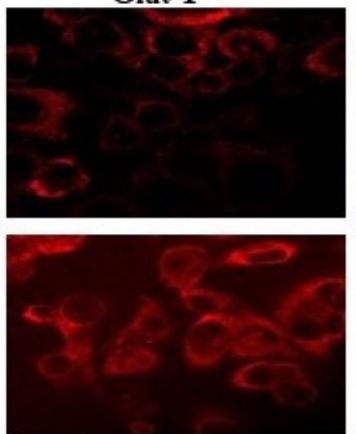

Merge
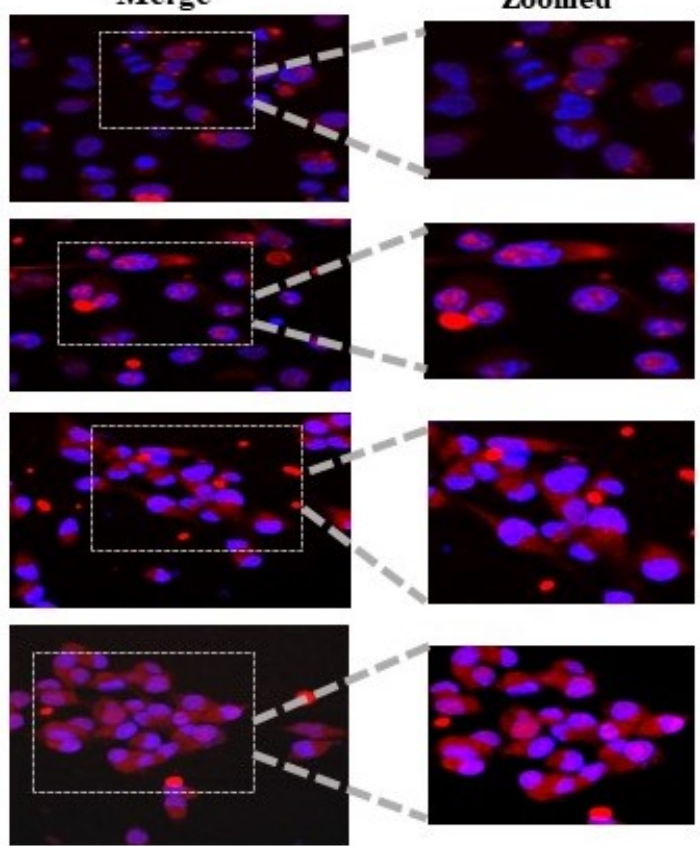

Merge

Zoomed
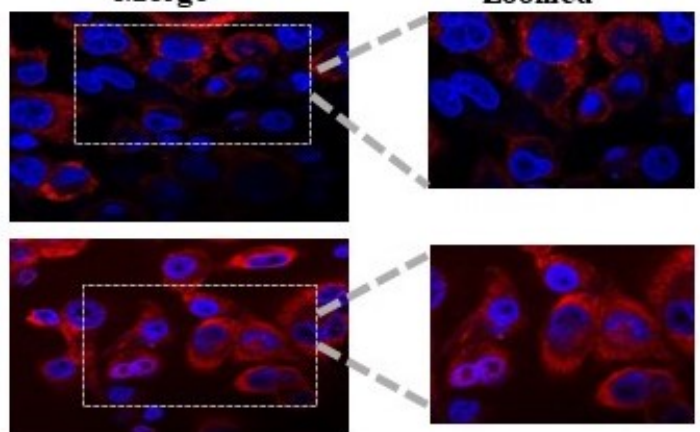

Figure 2-3. MUC13 Enhances Expression of c-Myc and Glut-1 in Cells Confocal microscopy images showing the expression of (A) c-Myc and (B) Glut-1 in indicated cells. Cells were seeded in 4-well chambered slides, fixed, permeabilized and stained with respective antibodies. The slides were further processed and analyzed for confocal microscopy. Images were captured at 400X. DAPI was used as a counter stain for the nucleus. 

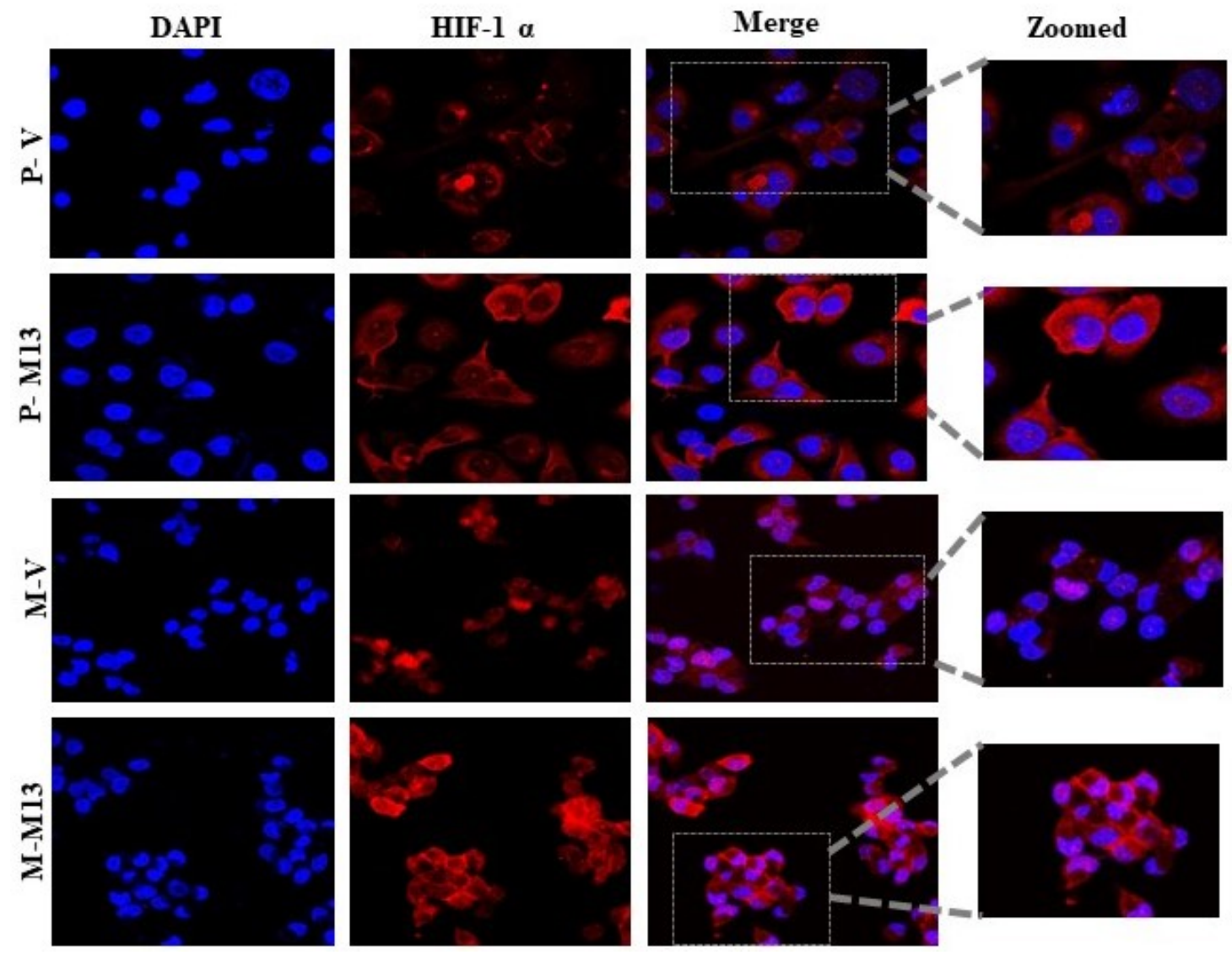

Figure 2-4. MUC13 Enhances Expression of HIF-1 $\alpha$ in Cells

Confocal microscopic images showing the expression of HIF-1 $\alpha$ in indicated cells. Cells were seeded in 4-well chambered slides, fixed, permeabilized and stained with HIF-1 $\alpha$. The slides were analyzed for confocal microscopy. DAPI was used as a counter stain for the nucleus. 


\section{MUC13 Expression Leads to the TNF-Induced Activation/Nuclear Shuttling of NFкB p-65}

We further wanted to investigate the signaling pathways that might be involved in the MUC13 influenced aberrant glucose metabolism in pancreatic cancer cells. Our results demonstrate that MUC13 expression facilitates TNF-induced activation/phosphorylation of NFKB-p65 at Ser536 and its nuclear translocation in Panc-1 and MiaPaca (P-M13 and M-M13) cells. Although, TNF- $\alpha$ induced p65 nuclear accumulation (0-30 min) was also observed in vector control cells (M-V and P-V), but a remarkable increase was observed upon MUC13 expression (M-13 and P-M13), suggesting its involvement in the MUC13 mediated signaling mechanisms (Figure 2-5A). These events were observed with concomitant degradation/decrease in the expression levels of I $\mathrm{B} \alpha$ (NFKB repressor) in the cytoplasm, which is ultimately known to release its inhibition from NFкB p-65 Western blotting depicting the effect of p65 silencing on the metabolism involved proteins in presence of TNF- $\alpha$ stimulation $(5 \mathrm{ng} / \mathrm{ml}$ for 24 hours $)$ accumulation and phosphorylation of NFKB p65 (Figure 2-5B) and accompany its translocation to the nucleus [154]. Similar results were corroborated on MUC13 knockdown in HPAF-II cells, which indicated decreased nuclear (Figure 2-5C) as well as cytoplasmic I $\mathrm{B} \alpha$ degradation in MUC13 knockdown cells (sh-M13) (Figure 2-5D). These results suggest the association of MUC13 to the promotion of TNF- $\alpha$ induced NFкB activation. These results indicate that MUC13 induces NF- $\kappa \mathrm{B}$ activation, which is independent of the mode of stimulation and may involve distinct mechanisms augmenting NF- $\kappa \mathrm{B}$ activation. One such mechanism may be similar to the one discussed previously in colon cancer, which suggests the role of MUC13 in augmented recruitment of cIAP1 to the TNFR/RIPK1 complex, and promotion of RIPK1 ubiquitination by

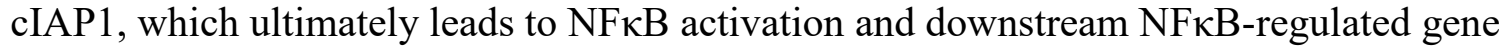
expression [155]. We then examined whether TNF- $\alpha$-induced I $\kappa \mathrm{B} \alpha$ degradation is due to $\mathrm{I} \kappa \mathrm{B} \alpha$ phosphorylation, and used a proteasome inhibitor, MG132 to block $\mathrm{I} \kappa \mathrm{B} \alpha$ degradation. Western blotting analysis revealed TNF- $\alpha$ induced phosphorylation/degradation of I $\kappa \mathrm{B} \alpha$ only in MUC13 expressing cells (M-M13) but not in vector control cells (M-V) (Figure 2-5E), which was further restored on MG132 treatment. These results confirm the role of MUC13 in the induction of $\mathrm{NF \kappa B}$ activation via $\mathrm{I} \kappa \mathrm{B} \alpha$ phosphorylation and degradation.

In order to determine if $\mathrm{NF}$ КB is required for MUC13 mediated alteration of glucose metabolism, we examined the expression of important proteins involved in regulation of glucose metabolism on silencing p65 in MUC13 null or stably expressing MiaPaca cells. Following TNF- $\alpha$ exposure for 24 hours, MUC13 expressing cells exhibited increased expression of c-Myc, HIF-1 $\alpha$, Bcl-2, and Glut-1 as compared to MUC13 null cells. This increase however, was repressed by silencing of p65 (Figure 2-5F). Also, we demonstrated that presence of MUC13 leads to the okadaic acid (another inducer of NFKB) led to the nuclear translocation of NFKB p-65 (Figure 2-6). These results altogether suggest that NFKB is required for MUC13 induced expression of proteins associated with enhanced glucose metabolism in pancreatic cancer cells. 


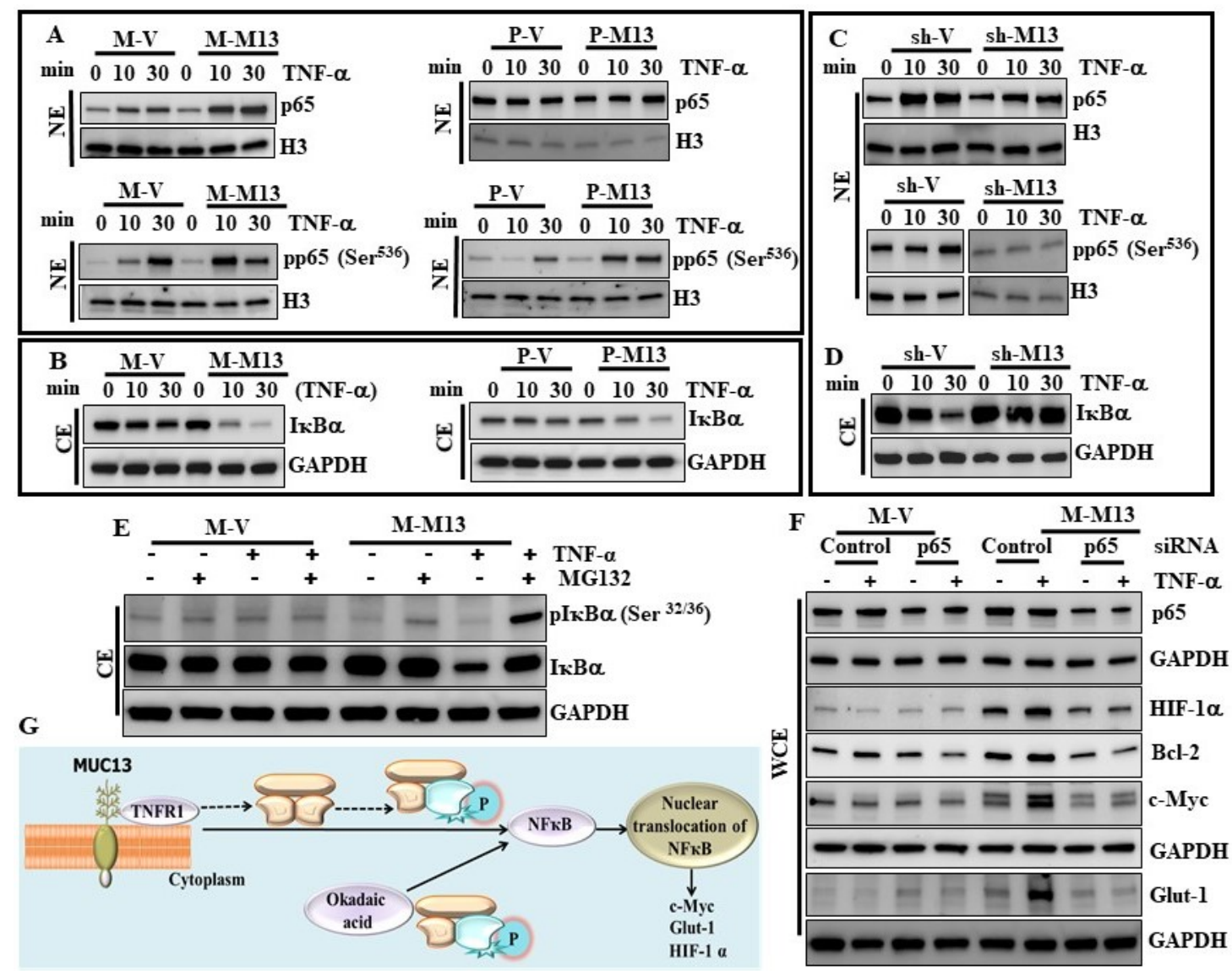

Figure 2-5. MUC13 Expression Leads to Enhanced Activation/Nuclear Translocation of NFKB p-65

(A) Western blots showing the expression level of p65 and phospho-p65 in M-V/M-M13 and P-V/P-M13 cells. Cells were spiked with TNF- $\alpha$ at a concentration of $20 \mathrm{ng} / \mathrm{ml}$ for 0 , 10 and 30 minutes, and the nuclear extracts prepared. Histone $\mathrm{H} 3$ served as the internal control. (B) Western blots indicating changes in the expression of I $\mathrm{B} \alpha$ shown in the cytoplasmic extracts in aforementioned cell lines. (C and D) Western blots showing the expression level of p65, phospho-p65 and I $\mathrm{B} \alpha$ in the nuclear and cytoplasmic extracts of MUC13 expressing (sh-V) and MUC13 knockdown (sh-M13) HPAF-II cells. (E) Western blotting depicting the degradation of $\mathrm{pI} \kappa \mathrm{B} \alpha$ and $\mathrm{I} \kappa \mathrm{B} \alpha$ after treatment with MG132 (1 hr, $10 \mu \mathrm{M})$ in presence of TNF- $\alpha$ stimulation $(20 \mathrm{ng} / \mathrm{ml}, 30$ minutes) (F) Western blotting depicting the effect of p65 silencing on the metabolism involved proteins in presence of TNF- $\alpha$ stimulation ( $5 \mathrm{ng} / \mathrm{ml}$ for 24 hours) (G) Schematic

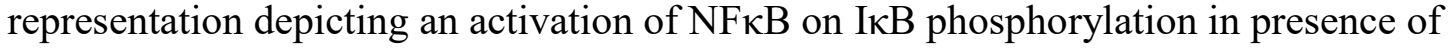
MUC13 following TNF- $\alpha$ or okadaic acid stimulation. 


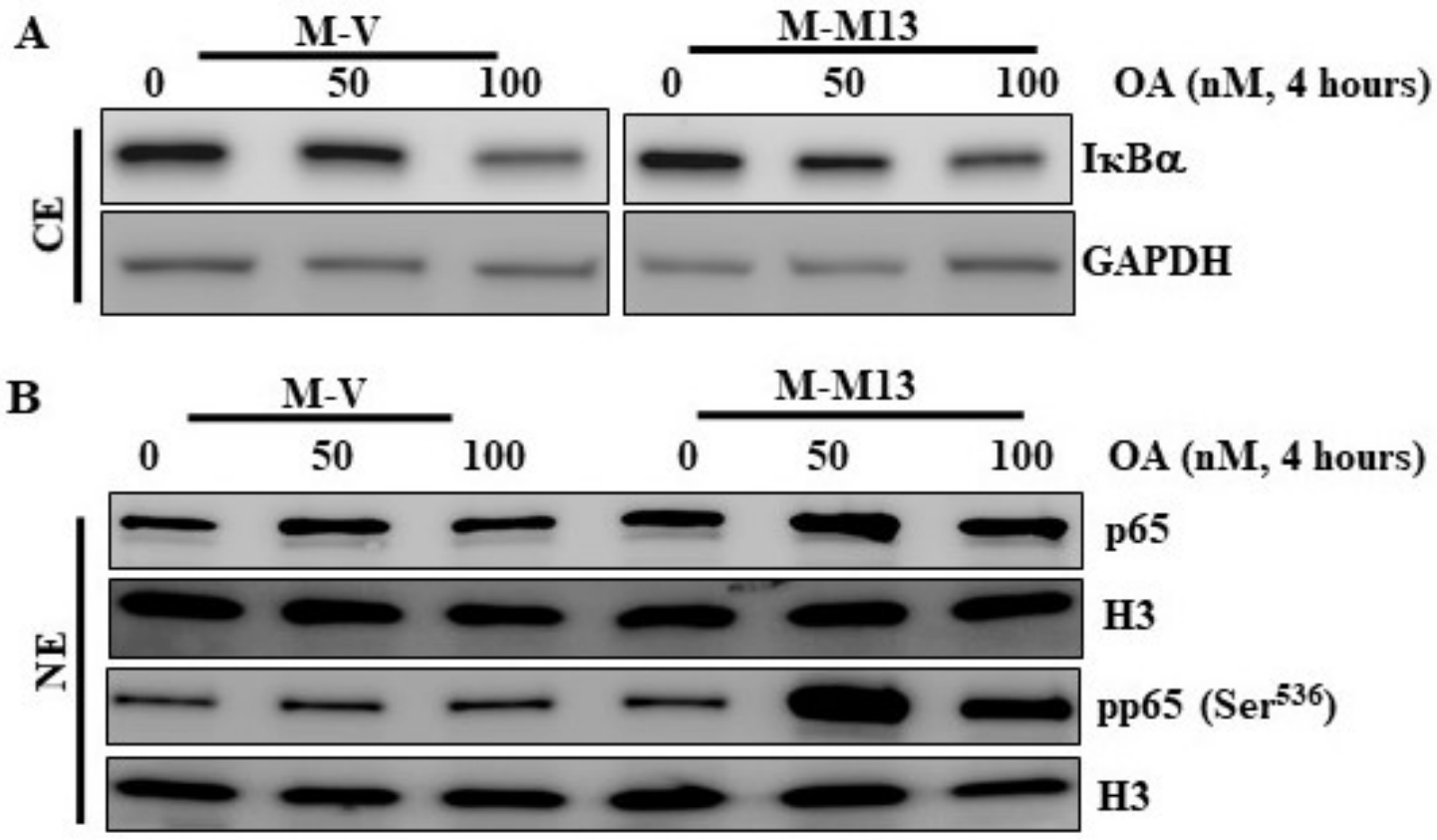

Figure 2-6. MUC13 Expression Leads to the OA-Induced Activation/Nuclear Translocation of NFкB p-65

(A) Western blots showing the expression level of I $\mathrm{KB} \alpha$ in cytoplasmic lysates from MiaPaca (M-V/M-M13) cells. Cells were stimulated with Okadaic acid at indicated concentrations $(0,50$ and $100 \mathrm{nM})$ for 4 hours. (B) Western blots showing the expression level of p65 and phospho-p65 in the nuclear lysates of aforementioned cells. Histone H3 served as the internal control. 


\section{MUC13 Interacts with Glut-1 and This Interaction Is Disrupted by NFאB Inhibition}

Our results demonstrate increased Glut-1 expression in Panc-1 and MiaPaca cells while MUC13 is exogenously overexpressed, thus it is warranted to investigate molecular cross talk between these proteins. Our investigations revealed a direct molecular interaction of MUC13 with Glut-1 in PDAC cells as demonstrated by multi-level experimental techniques, such as reciprocal co-immunoprecipitation (co-IP), proximity ligation assay (PLA), co-capping and co-localization assays (Figure 2-7A through F).

Results from reciprocal co-IP showed that MUC13 and Glut-1 remains in a molecular complex. This interaction stabilizes Glut-1 as demonstrated by cycloheximide chase assay with half-life as low as $1 \mathrm{hr}$ in MUC13 null P-V cells and greater than 3 hours in MUC13 expressing P-M13 cells (Figure 2-7C. D).

Proximity ligation assay demonstrated that the interaction between these proteins is direct as both proteins exist in a close proximity as indicated by multiple red spots on the cell surface (Figure 2-7E). MUC13 with HER2 serve as an experimental positive control in PLA as they are reported to physically interact in PDAC cells. Additionally, co-capping assay showed a progressively increasing distribution of both MUC13 and Glut-1 staining into membrane caps which strongly suggests their co-clustering (yellow color) and molecular interaction in PDAC (Figure 2-7F). Co-capping experiment, performed with $\alpha$-tubulin in HPAF-II cells, did not show any clustering with MUC13, and served as negative experimental control. We further confirmed our observations through confocal immunofluorescence utilizing antibody-mediated double immunofluorescence staining technique. Results of this analysis showed strong colocalization (yellow color) of MUC13 and Glut-1 in both HPAF-II and AsPC1 cells (Figure 2-8A). Interestingly, this co-localization was abrogated upon treatment with a pharmacological inhibitor of NFкB, sulphasalazine (Figure 2-8B). Sulphasalazine is known to inhibit NFкB activation/translocation to nucleus via direct inhibition of enzyme ІкВ kinase (IKK) that phosphorylates/inhibits NFкB repressor, ІкВ $\alpha$ [156]. Similar results were obtained in reciprocal co-IP in which MUC13 expressing PDAC cells failed to form MUC13-Glut-1 molecular complexes in presence of sulphasalazine (Figure 2-8C, D). We further sought to investigate whether the interaction between the two proteins and NFкB activation influence MUC13 mediated altered glucose metabolism in PDAC cells. We observed reduced lactate production in MUC13 expressing cells on treatment with sulphazalazine (Figure 2-8E). These results imply that molecular interaction between MUC13 and Glut-1 is mediated through NFKB activation and suggest the role of MUC13 in glucose metabolism via alteration of Glut-1 functionality in PDAC cells.

\section{MUC13 and Glut-1 Expression Directly Correlate in Pancreatic Cancer Tissues}

To understand clinical correlation of MUC13-Glut-1 molecular interaction, we examined human pancreatic cancer tissues for MUC13 and Glut-1 expression using

qPCR technique (Figure 2-9 A, B). Two independent sets of samples were utilized for 


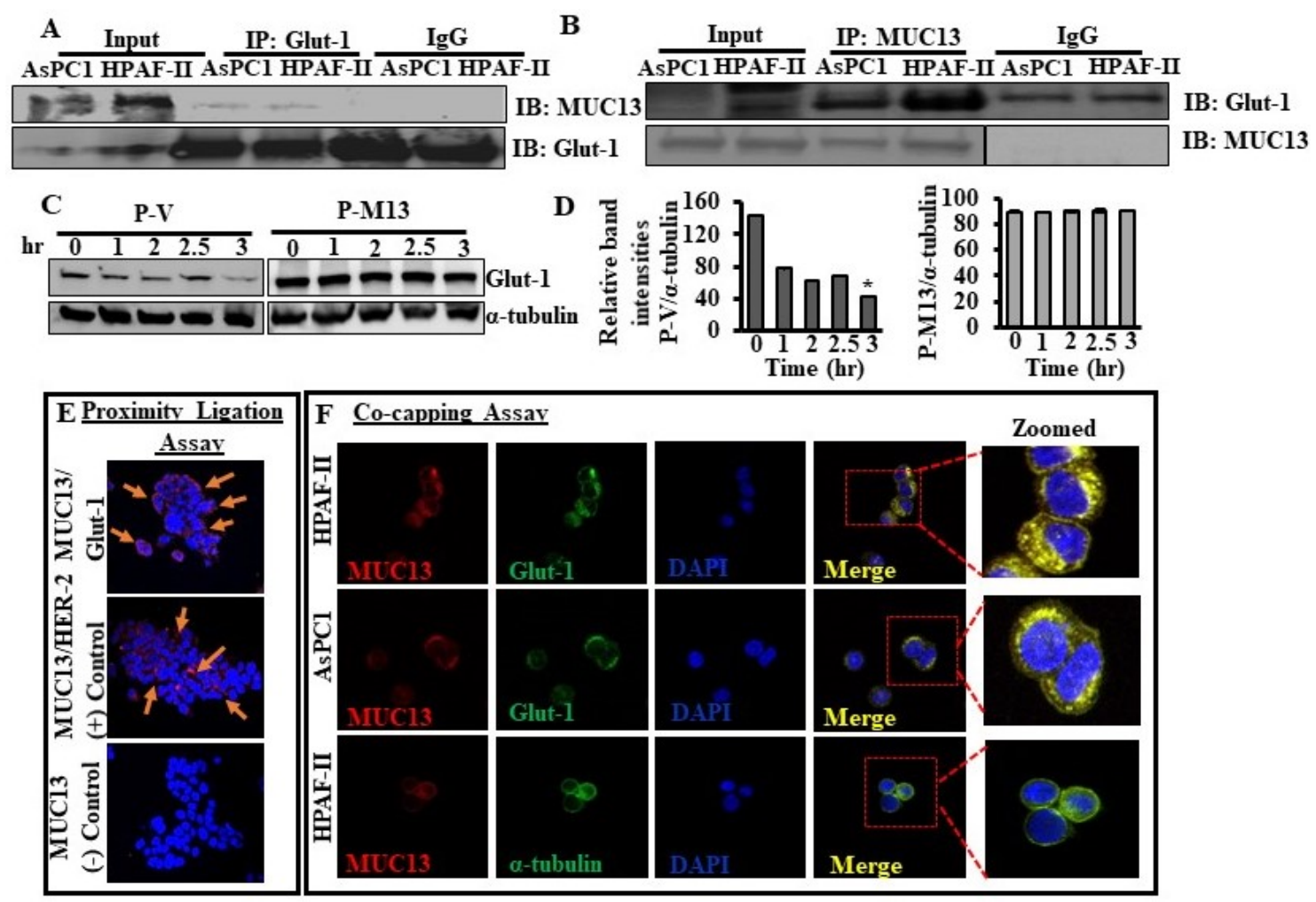

Figure 2-7. MUC13 Interacts with Glut-1 Protein

(A and B) Reciprocal co-immunoprecipitation assay performed between MUC13 and Glut-1 using whole cell protein lysates from MUC13 expressing, AsPC1 and HPAF-II cells. (C) Cycloheximide chase assay depicting the stability of Glut-1 in P-V and P-M13 cells. Cells were treated with cycloheximide $(10 \mu \mathrm{M})$ at different indicated time points followed by lysate preparation and Western blotting. Alpha-tubulin ( $\alpha$-tubulin) served as an internal control. (D) Bars representing relative quantification of the blots. (E) In situ proximity ligation assay (PLA) performed using HPAF-II cells with the Duolink Red starter kit. DAPI was used as a counter stain for the nucleus. MUC13/Her2 served as positive control, and MUC13 alone as negative control. The co-localization between MUC13/Glut-1 and MUC13/Her-2 has been indicated by arrows. (F) Co-capping assay to demonstrate interaction between MUC13 and Glut-1 in HPAF-II and AsPC1 cells. HPAF-II cells with anti- $\alpha$ tubulin is a negative control for this experiment. Images were captured at $400 \mathrm{X}$. 


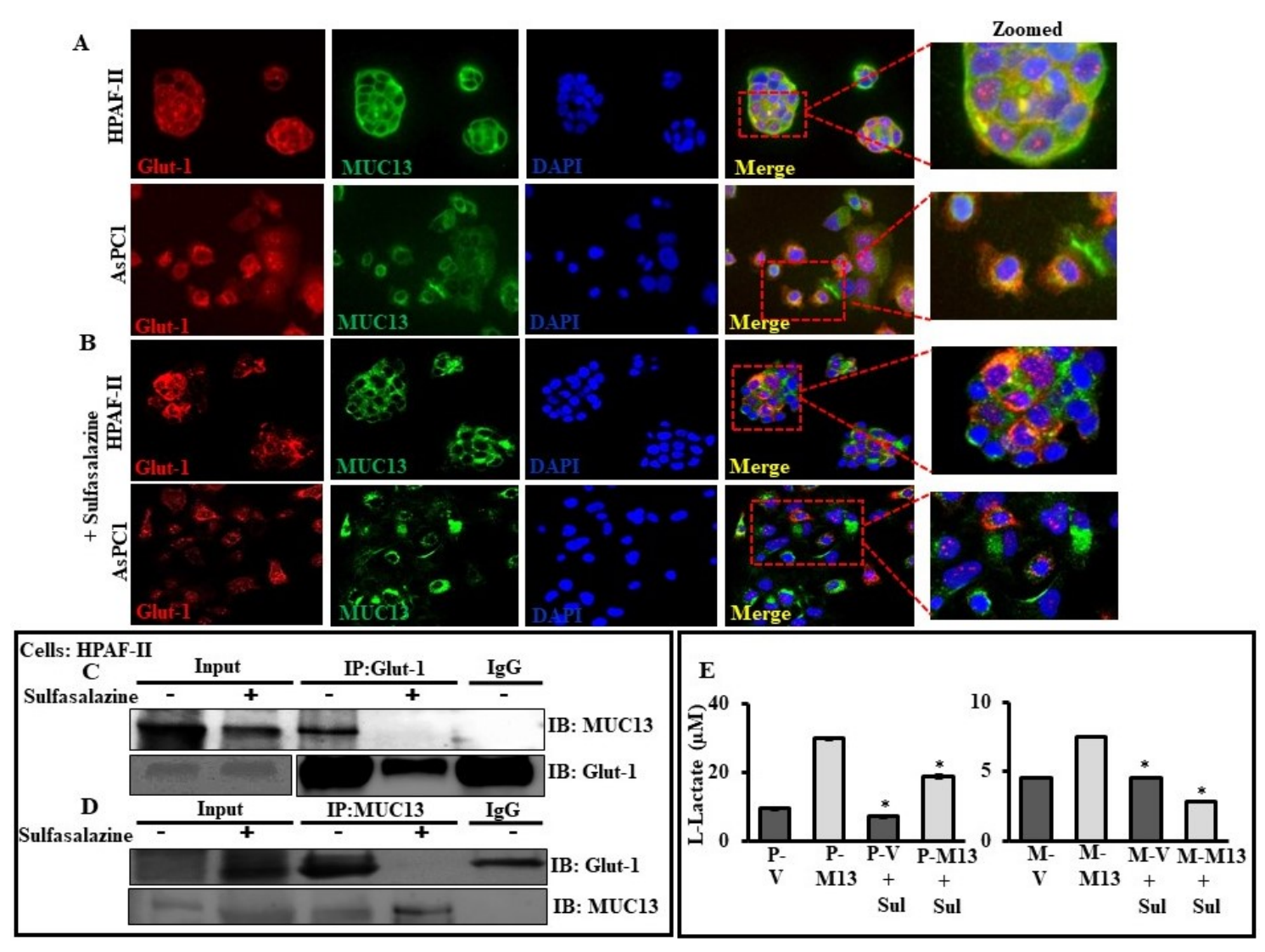

Figure 2-8. MUC13-Glut-1 Interaction is Disrupted by Sulfasalazine

(A) Confocal immunofluorescence of MUC13 and Glut-1 in MUC13 expressing, HPAFII and AsPC1 cells depicting co-localization (yellow color). Cells were cultured in 4-well chambered slides, fixed, permeabilized, stained with indicated antibodies and analyzed for confocal microscopy. DAPI was used as a counter stain for the nucleus. (B) Cells were treated with sulphasalazine $(0.9 \mathrm{mM})$ for $24 \mathrm{hrs}$ and processed for immunostaining and confocal microscopy. Images were captured at 400X. (C and D) Reciprocal coimmunoprecipitation assay between MUC13 and Glut-1 performed using protein lysates from HPAF-II cells. Cells were treated with sulfasalazine $(0.9 \mathrm{mM})$ for 24 hours and protein lysates prepared. The immunoprecipitates were resolved on a $10 \%$ gel, and followed by immunoblotting with anti-MUC13 and anti-Glut-1 antibodies. (E) Lactate assay was performed in MUC13 expressing and null cells after treatment with or without sulfasalazine. Cell culture media was collected after 48 hours to measure the amount of L-Lactate concentration using lactate assay kit. 

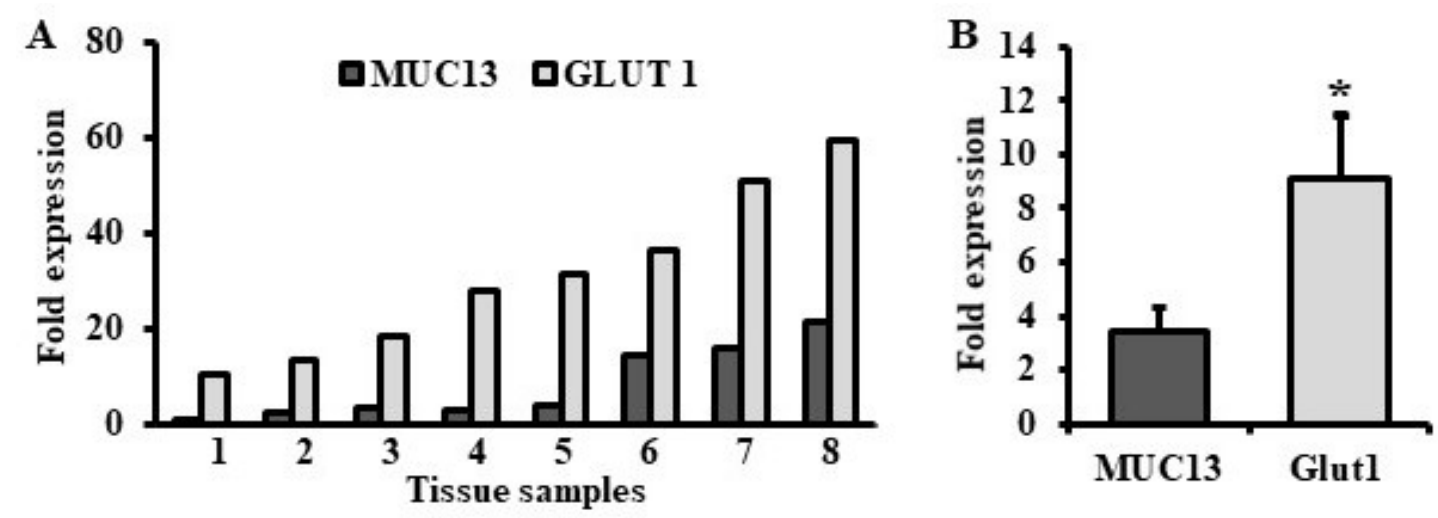

C

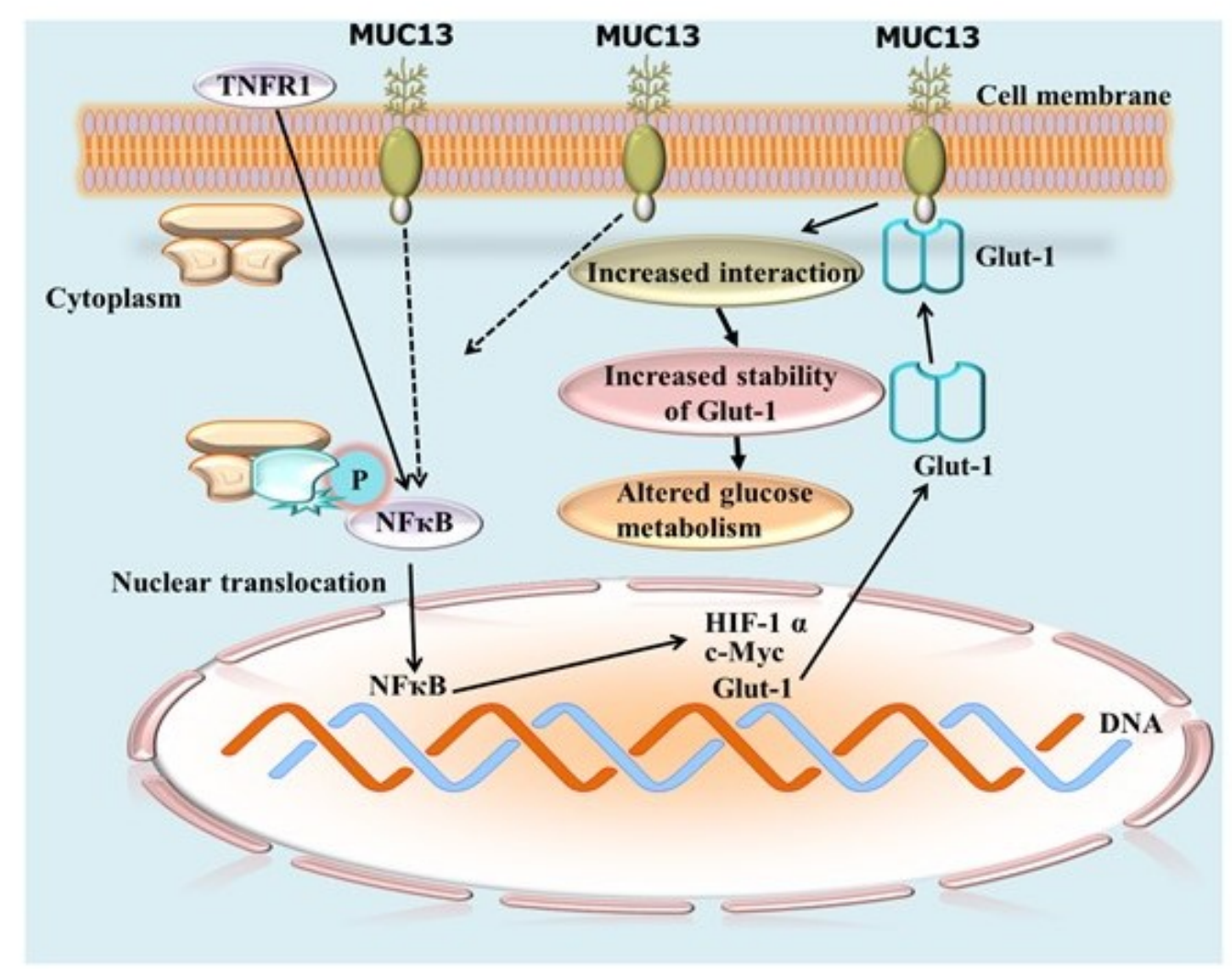

Figure 2-9. MUC13 and Glut-1 Expression Directly Correlate in Human Pancreatic Cancer Tissues

(A) qPCR was performed to analyze the levels of MUC13 and Glut-1 using RNA from freshly collected human PDAC tissues $(\mathrm{N}=8)$ procured from Baptist Memorial hospital, Memphis. (B) and cDNA from PDAC tissues $(\mathrm{N}=6)$ procured from Origene. The correlation coefficient $(r)$ and coefficient of determinations ( $(r 2)$ were 0.982 and 0.862 respectively. The analysis was performed by graph pad prism software. (C) Schematic representation for the mechanism of MUC13 mediated glucose metabolism. 
the experiment, which include freshly collected RNA from PDAC tissue samples $(\mathrm{N}=8)$ procured from Baptist Memorial hospital, Memphis (Figure 6A); and cDNA (N=6) procured from Origene (Figure 2-9B). Analysis of MUC13 and Glut-1 expression in both the sets revealed a positive correlation between the expressions of these genes in human pancreatic cancer tissues. All tissue samples were positive for MUC13 and Glut-1, which showed a similar trend in the expression levels. The correlation coefficient (r) and coefficient of determinations (r2) were 0.982 and 0.862 respectively. The analysis was done using graph pad prism software.

\section{Discussion}

High cellular glucose metabolism has been recently recognized as one of the hallmarks of cancer. Pancreatic cancer cells especially are highly active metabolically and programmed for extensively higher consumption of bio-fuels to operate oncogenic machinery. Increased glucose consumption of pancreatic cancer cells to meet the pathological requirements is supported by Warburg effect. This rewired metabolism is acquired to support their rapid proliferation and metastasis across the body. Although, several oncogenes and tumor suppressors are known to facilitate the metabolic adaptation in pancreatic cancer [157], investigation of novel molecular targets and their mechanisms are highly essential to improve treatment outcomes of this highly dreadful disease. In this study, we observed the mechanistic role of MUC13 as an oncogenic molecular target that modulates glucose metabolism in pancreatic tumor cells. Our study provides the first evidence for the critical involvement of MUC13 in metabolic reprogramming of pancreatic cancer cells by activating the NFKB survival pathway and molecular interaction with Glut-1 receptor.

Our results demonstrate molecular mechanisms that drive the glycolytic phenotype conferring growth advantage to pancreatic cancer cells. This signifies a critical role of MUC13 in tumor microenvironment favoring the development of a more aggressive and invasive phenotype. Our study presents three important conclusions implicating the mechanisms that stimulate increased glucose metabolism and lactate production in pancreatic tumors. Our data firstly demonstrates that MUC13 expression alters the metabolic phenotype of pancreatic cancer cells and influences their proliferation and invasiveness due to enhanced lactate secretion. This as a result, provides an acidic tumor microenvironment, that contributes to highly aggressive and metastatic behavior of cancer cells via influencing a number of oncogenic signaling pathways, including the activation of proteins that regulate glucose transport [158]. One such main protein is Glut-1, which represents a potential therapeutic target and its expression level is altered with MUC13 expression. 2-Deoxyglucose, a metabolic inhibitor of Glut-1 receptors, inhibits up to 59-95\% growth of PDAC cells [159]. HIF-1 $\alpha$ levels in MUC13 expressing cells increase under normoxic and hypoxic environments accompanied by increase in lactate production. These results suggest that MUC13 induced altered glucose metabolism involves the activation of important proteins, such as Glut-1 and HIF-1 $\alpha$, which may be contributing to MUC13 mediated adaptive cellular mechanisms in tumor microenvironment to maintain a glycolytic phenotype. Secondly, our investigations 
reveal MUC13 induced activation of NFאB survival pathway, which triggers a number of key proteins involved in altered glucose metabolism. Enhanced ІкВ $\alpha$ degradation and activation/nuclear translocation of NFאB in MUC13 expressing cells was observed following TNF- $\alpha$ or okadaic acid stimulation. These events were typically accompanied by activation of c-Myc, HIF-1 $\alpha$ and Glut-1 expression levels that are observed to be abrogated specifically in MUC13 cells following silencing of NFKB. Our data indicates that the metabolic events controlled by MUC13 occur following NFאB activation.

MUC13 has been recently reported to functionally interact with HER2 and this interaction mediates MUC13 induced pancreatic cancer progression. This study elucidated a novel, MUC13-Glut-1 interaction that may be one of the important events in driving mechanisms of aberrant glucose metabolism in pancreatic cancer cells. We found that the interaction between MUC13 and Glut-1 is governed by constitutive NFKB activation. MUC13 interaction stabilizes Glut-1 as suggested by increased half-life of Glut-1 in MUC13 expressing cells compared to MUC13 null cells. Interaction between these two proteins appears to be direct and in very close proximity, as evident by co-IP and PLA assays, respectively. Since MUC13 and Glut-1 complex is dependent on nuclear translocation of NFאB as it is prevented by sulphasalazine treatment, suggest an opportunity to develop novel therapeutic strategies to attenuate aggressive/metastatic behavior of pancreatic cancer cells. However, it also leads to questions, as to how NFkB activation promotes interaction between MUC13 and Glut-1, and which specific domains of both proteins are involved in this molecular interaction. Although, further investigations are warranted, we speculate that a cytoplasmic domain of MUC13 binds to Glut-1, as cytoplasmic domain of MUC13 is suggested to have role in cell signaling [160]. A positive correlation between the expression of MUC13 and Glut-1 in human pancreatic cancer tissues suggest clinical correlation of our proposed novel mechanism related to aberrant glucose metabolism in pancreatic tumors.

In summary, this study revealed a novel role of MUC13 in rewiring of a distinct glucose metabolic network that drives favorable tumor microenvironment and oncogenic signaling pathways in pancreatic cancer cells for the adoption of their enhanced tumorigenic and metastatic behavior. Thus, inhibition of MUC13 via conventional pharmacological agents and/or non-conventional siRNA/miRNA mediated technologies can selectively suppress pancreatic tumor growth and metastasis via inhibition of glycolytic influx in pancreatic cancer cells. Our observations suggest that the reliance of pancreatic tumor growth and metastasis on MUC13-Glut-1 mediated glucose metabolism can be exploited for the development of novel targeted therapeutic strategies for pancreatic cancer. Finally, our study offers a very promising target for therapeutic manipulation via disabling aberrant glucose metabolism in pancreatic cancer patients, thereby prolonging survival rate of patients suffering from this dreadful disease. Next, it was essential to identify other molecular targets which are involved in metabolic reprogramming leading to invasive pancreatic cancer. In this regard, we further investigated the role of another protein, Protein Kinase D1 (PKD1), in aberrant glucose metabolic network in pancreatic cancer, which has already been reported to have a role in pancreatic tumorigenesis. 


\section{CHAPTER 3. PROTEIN KINASE D1 INDUCES METABOLIC SWITCH IN PANCREATIC CANCER VIA MODULATION OF MAMMALIAN TARGET OF RAPAMYCIN}

\section{Introduction}

Pancreatic cancer is a highly malignant disease, and prevalent in the United States that ranks third in mortality rates related to cancer [1]. The lack of early detection, chemoresistance and increased metastasis contributes to the highly aggressive behavior of this disease and poor survival rate [1]. Therefore, it is essential to investigate and comprehend mechanisms that lead to the tumor aggressiveness and chemoresistance in pancreatic cancer. One such unique characteristic physiology of pancreatic tumors includes a hostile and tumor microenvironment, which favors biochemical and metabolic adaptations to facilitate pancreatic tumor growth and metastasis. The metabolic alterations enable cells to satisfy their bioenergetics needs by adapting to the changing environments for survival, longevity and availability of nutritional requirements[161]. Cellular signaling empowers cancer cells to acquire and process nutrients in a manner favorable to proliferation than production of ATP [162]. Through aerobic glycolysis, cancer cells produce energy by converting glucose into lactate, in presence of oxygen, which results in enhanced synthesis of lipids and nucleotides and stimulates growth and invasion. Herein, we postulate that the identification and strategic targeting of molecules that regulate altered glucose metabolism can help in the effective maintenance of the disease and development of therapeutics.

Protein Kinase D1 (PKD1), a serine-threonine kinase, is an important modulator of several kinase signal transduction pathways [163]. PKD1 belongs to the PKD family and is the most studied protein among the three family members (PKD1, PKD2 and PKD3) [163]. Previous reports have suggested that PKD1 is involved in pancreatic cancer pathogenesis $[88,164]$, but the underlying signaling mechanisms are largely unknown. Studies have suggested the role of PKD1 in increasing pancreatic cancer proliferation and growth rate through stimulation of $\mathrm{NF \kappa B}$, an important transcription factor involved in various cellular mechanisms [165]. PKD1 has also been reported to be involved in the Notch pathway being downstream of KRAS, leading to pancreatic neoplasia [164]. PKD1 also regulates the duct formation leading to pancreatic cancer initiation and growth [166]. PKD1 acts as one of the reactive oxygen species (ROS) sensing molecule, increases oxidative stress, ultimately resulting in pancreatic cancer progression [88]. Therefore, we were interested to investigate the mechanisms by which PKD1 may contribute to pancreatic tumorigenesis and understand the signaling processes involved therein.

One of the most important pathway regulating metabolic processes is mTOR (mammalian target of rapamycin) pathway [167]. mTOR is a serine/threonine kinase that exists as two different complexes, mTORC1 and mTORC2. mTOR, in association with raptor and $\mathrm{mLST} 8$ forms $\mathrm{mTORC1}$, regulates protein translation, cell-cycle progression 
and metabolism [161]. While, mTOR, in association with Rictor (rapamycin insensitive companion of mTOR) and mSIN1 (stress-activated protein) forms mTORC2 and regulates cell survival/glucose metabolism and cytoskeletal remodeling [167].

Rapamycin is known to inhibit mTORC1 activity and promotes $\beta$-oxidation for anabolic storage pathways.

Therefore, in this study we suggest a novel role of PKD1 in regulating glucose metabolism in pancreatic cancer, which drives pancreatic tumorigenesis, chemoresistance and progression. This takes place in correlation with mTOR signaling, wherein mTORC1 predominantly regulates PKD1 induced metabolic changes. Briefly, our study prospects PKD1 as a novel molecular target in pancreatic cancer for therapeutic intervention.

\section{Materials and Methods}

\section{Cell Culture}

Pancreatic cancer cells, HPAF-II/BxPC3 (low PKD1 expression); Panc1/AsPC1/Capan-1/MiaPaca (high PKD1 expression) were used for this study. All cells were maintained in DMEM/F12 or RPMI media containing $10 \%$ fetal bovine serum in an incubator at $37^{\circ} \mathrm{C}$ temperature with $5 \% \mathrm{CO}_{2}$. The normal human pancreatic cell line, HPNE, was maintained in DMEM media. The complete media, and the cell lines were

maintained. The cells were frequently investigated for mycoplasma growth and contamination.

\section{Procurement and Scoring of Human Pancreatic Cancer Tissues for Analysis of PKD1 Expression}

Pancreatic cancer tissue microarray from US Biomax (\# BIC14011a SL160) with chronic pancreatitis $(n=12), \operatorname{PanIN-1}(n=10), \operatorname{PanIN-2}(n=8)$ and PDAC $(n=8)$. Also, normal $(n=3)$ tissues were obtained from Baptist Memorial Hospital $(\mathrm{BMH})$, Memphis, TN. The scoring of the PKD1 expression was performed as described earlier in detail [84] . Stained slides were digitally scanned and analyzed for PKD1 staining at the UTHSC Pathology Department. Captured images were independently analyzed and scored by three reviewers, who were kept blinded to the patient history. The intensity of immunoreactivity of PKD1 was graded on a 0 to 4 scale ( 0 for no staining, 1 for weak immunoreactivity; 2 for moderate immunoreactivity; 3 for strong immunoreactivity, and 4 for very strong immunoreactivity). The percentage of cells positive for PKD1 immunoreactivity within the tumor and normal tissue sections were scored as follows: 0 $25 \%$ as $1,26-50 \%$ as $2,51-75 \%$ as 3 and $76-100 \%$ as 4 . The mean composite score (MCS) was determined by calculating an average of the composite scores (intensity $\mathrm{x}$ percentage) of respective samples in each category. 


\section{Western Blotting}

The whole cell lysates from pancreatic cancer cells were prepared and used for investigating the changes in protein expression through Western blotting procedure, as described before $[11,168]$. Briefly, $40 \mu \mathrm{g}$ of protein was loaded per well to perform SDS PAGE followed by Western blotting. The transfer of gels was done on PVDF (polyvinylidene difluoride) membrane and membranes blocked in either 5\% non-fat dairy milk or 5\% BSA (bovine-serum albumin for 1 hour followed by incubation with the primary antibodies overnight at $4^{\circ} \mathrm{C}$. The primary antibodies with the following catalog numbers were procured from Cell Signaling Technology: HIF-1 $\alpha$ (3716), Glut-1 (12939), Ras (3965), KRAS-12D (14429), eIF4e (9742S), peIF4e (9741S), 4EBP1 (9452S), p4EBP1 (2855S), mTOR (4517S), and p-mTOR (5536S). Beta-actin antibody was obtained from Sigma (A2228). The rabbit (4011), and mouse (4021) secondary antibodies were purchased from Promega company.

\section{Immunofluorescence}

Immunofluorescence was conducted as described earlier [150]. Briefly, cells were fixed, permeabilized and incubated overnight with primary antibodies. Following day, the cells were incubated with respective secondary antibodies for 1 hour, washed and mounted. Further, the images were taken under a confocal microscope at $400 \mathrm{X}$ magnification (Zeiss 710, Germany).

\section{Immunohistochemistry}

Pancreatic cancer human tissues microarrays were obtained from Baptist Memorial Hospital, Memphis and were immunostained using antigen-retrieval method, as described earlier [84] using commercially available Biocare kit from Biocare Medical, CA, USA,. Briefly, the tissue slides were deparaffinized, rehydrated, peroxidized and antigen retrieved. Further, the tissues were incubated with primary antibodies overnight followed by addition of secondary MACH4 Universal HRP mouse polymer (\#M4U534H) followed by staining with 3-diaminobenzidine (DAB) chromogen (\#DB801) and counterstaining with hematoxylin. The slides were then subjected to dehydration steps and mounting [83].

\section{Transfection of Cells}

Pancreatic cancer cells having low PKD1 expression (HPAF-II and BxPC3) were incubated in serum-free OPTI-MEM media overnight, and transiently transfected with PKD1 expression plasmid and control pEGFP using Lipofectamine 2000 reagent. Following six hours of transfection, $10 \%$ serum-containing regular media was added to the cells after removing the old media. The transfection procedure was terminated after 48 hours and the cells were used for further experiments. 


\section{Lactate Assay/ Glucose Assay}

Lactate and Glucose assays were performed by utilizing commercially available kits from Cayman Chemicals (lactate assay kit: catalog No.: 600450 and glucose assay kit: catalog No. 10009582). Cells were seeded as per the manufacturer's instructions in the kit, and media of cells was used to determine the amount of lactate production and glucose consumption upon termination of the experiment at 24 and 48 hours. Further, the absorbance was recorded at the indicated wavelength, and readings were analyzed/calculated.

\section{Cell Migratory Assay}

The cell migration assay was conducted using Boyden chamber plate $[11,169$, 170]. 50,000 pancreatic cancer cells were seeded on the top chamber of the 96 well plate in a serum-free media, and $250 \mu 1$ of serum-containing media was added to the lower chamber. L-Lactate $(2 \mathrm{mM})$ or 2-Deoxyglucose $(10 \mathrm{mM})$ were added to the cells, which was followed by incubation for 18 hours at $37^{\circ} \mathrm{C}$. Thereafter, the cells were fixed with $4 \%$ paraformaldehyde, stained with crystal violet and washed with water. The images of cells were captured after complete drying of the plate.

\section{Cell Invasion Assay}

Cell invasion assay was performed using Matrigel Invasion Chambers from BD Biosciences [149]. 25,000 cells were seeded in the chambers containing media without serum in the upper chamber and complete media (10\% FBS) in the lower one. L-Lactate $(2 \mathrm{mM})$ or 2-Deoxyglucose $(10 \mathrm{mM})$ were added to the cells, which was followed by incubation for 24 hours at $37^{\circ} \mathrm{C}$. Further, the cells were fixed with methanol and crystal violet stained. The plates were washed with water and left to dry, which was followed by capturing images.

\section{Cell Proliferation Assay by MTT}

MTT assay was performed in PKD1 expressing and null cells to determine the effect of PKD1 in cell proliferation as described before [11]. Following 48 hours, $20 \mu 1$ MTT reagent was added to the cells. Then, 2 hours after the addition of this reagent, 100 $\mu l$ of dimethyl-sulfoxide (DMSO) solution was added on top of the cells and the plate was subjected to vigorous shaking for another 10 minutes. Next, the absorbance was read at a wavelength of $570 \mathrm{~nm}$, the cell viability was calculated and graph was plotted. 


\section{Clonogenic Studies}

The pancreatic cancer cells were seeded in 12-well plate ( 250 cells/well). The cells were subjected to both normoxic and hypoxic conditions at the same time. The experiment was terminated after 7 days, after PBS wash, adding fixative (cold methanol) for 10 minutes, and staining by crystal violet. This was followed by removal of stain, washing with water, drying of plate and capturing images.

\section{Gene Expression Studies}

RNA samples were isolated from various pancreatic cancer cells using Qiagen kit. The reaction was conducted as described earlier [11]. The concentration of samples was determined and $2 \mu \mathrm{g}$ of RNA was reverse transcribed. The expression of genes (PKD1, HIF-1 $\alpha$ and Glut-1) was determined by precise primers through real-time PCR experiment. GAPDH served as an internal control.

\section{Statistical Analysis}

The significance of the data in this manuscript was determined through Student's $t$ test, and $p$-values below 0.05 was set to be statistically significant.

\section{Results}

\section{PKD1 Is Overexpressed in Human Pancreatic Cancer Tissues and Cells}

The expression profile of PKD1 in human pancreatic cancer versus normal pancreatic cells was investigated using immunohistochemistry technique. PDAC and normal pancreas tissues were immuno-stained for PKD1 and scored. We evaluated the staining scores in MCS (mean composite score) taking in account of average staining pattern. Normal pancreatic tissues showed none or very faint PKD1 expression, whereas all of PDAC tissues showed moderate to high PKD1 expression (Figure 3-1A). Overall, PKD1 expression was significantly $(\mathrm{P}<0.0001)$ higher in PDAC compared to normal pancreatic ductal epithelium. PKD1 expression was predominantly expressed all over the malignant area of the pancreatic cancer tissues. Our results clearly indicate that PKD1 expression effectively differentiated PDAC from healthy controls.

The Mean Composite Score (MCS) was calculated for both normal and pancreatic cancer tissues, and was obtained by multiplying percentage intensity and percentage of tissue staining (Figure 3-1B and 3-1C). These results clearly suggest that PKD1 is overexpressed in pancreatic cancers and might have an oncogenic role in the malignant transformation of pancreatic cells. 

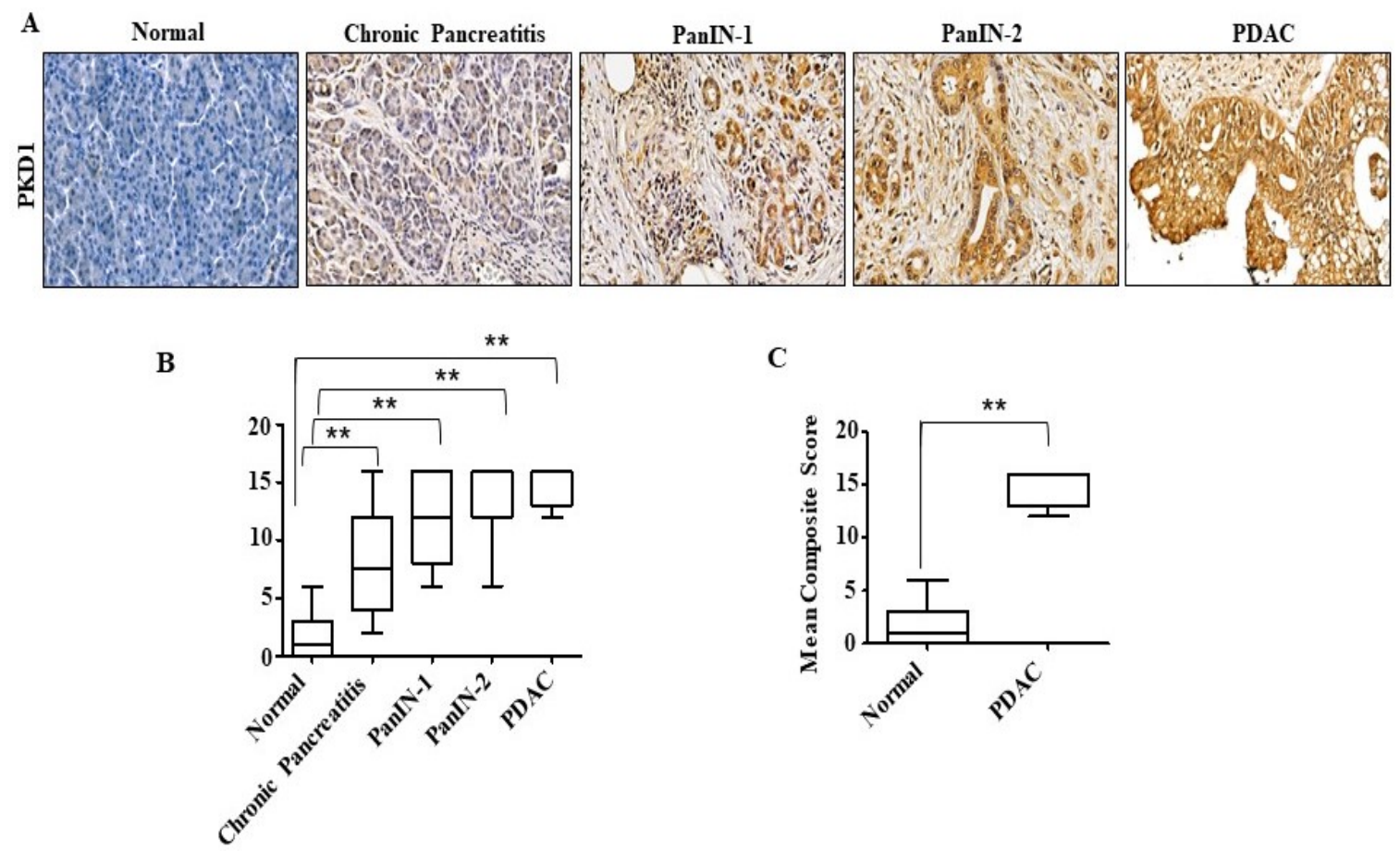

C

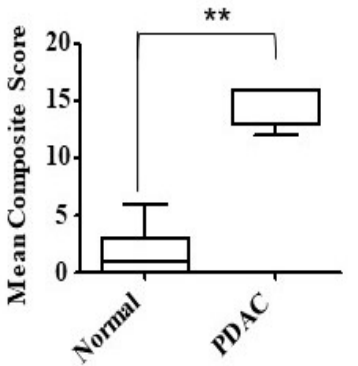

Figure 3-1. PKD1 is Highly Expressed in Human Pancreatic Cancer Tissues (A) Immunohistochemistry showing the expression of PKD1 protein in normal and various stages of pancreatic cancer human tissues. (B) Graph showing the mean composite scores of PKD1 staining in normal pancreatic tissues and the different stages of pancreatic cancer. (C) Quantification and depiction of the mean composite score in overall normal and pancreatic cancer tissues. $* * p<0.0001$. 
We also investigated the expression level of PKD1 in 6 pancreatic cancer cells, which includes, HPAF-II, Panc-1, Capan-1, MiaPaca, AsPC1 and BxPC3 cells. Except HPAF-II and BxPC3, which showed less expression of PKD1, most pancreatic cancer cells had high expression of PKD1 at both protein (immunoblotting and confocal immunofluorescence, (Figure 3-2A, B) and mRNA levels (PCR) (Figure 3-2C).

\section{PKD1 Contributes to Tumorigenic Characteristics via Glucose Modulation}

PKD1 overexpression or activation has been reported to facilitate survival and proliferation of pancreatic cancer cells [171]. Additionally, PKD1 is involved in trans differentiation of pancreatic acinar cells to a duct-like, pluripotent progenitor cell type (ADM, acinar-to- ductal metaplasia), leading to the initiation of pancreatic cancer[172]. Although, studies suggest the oncogenic role of PKD1 in pancreatic cancer, the precise mechanisms directly influencing the PKD1 induced tumorigenic characteristics are not elucidated. These studies are essential to develop strategies to control malignant transformation as well as increased tumor growth in pancreatic cancer. To investigate the underlying molecular mechanisms, we generated PKD1 overexpressing HPAF-II and BxPC3 cells (having low PKD1) using transient transfection. The transient transfection was performed to observe the abrupt molecular changes in the cell on altering the PKD1 expression. We observed an increase in the protein and mRNA levels of PKD1 expression in the both transfected cells, HPAF-II and BxPC3 using immunoblotting and PCR, respectively (Figure. 3-3A, B). We observed an increase in the percentage of cell viability on overexpression of PKD1 in both cells, HPAF-II and BxPC3 (Figure 3-3C). In order to investigate the role of PKD1 on glucose modulation in pancreatic cancer cells, glucose and lactate assays were performed in PKD1 overexpressing cells, HPAF-II and BxPC3. We observed significantly $(\mathrm{p}<0.05)$ higher upregulation of glucose consumption and L-lactate production in PKD1 overexpressing cells as compared to the control cells having basal levels of PKD1 (Figure. 3-4A, B). Therefore, these results indicate that PKD1 contributes to altered glucose metabolism of pancreatic cancer cells. Our results further demonstrated increased migration (Figure. 3-5A, B). and invasive (Figure. 3-6A, B) potential of HPAF-II and BxPC3 cells with PKD1 overexpression as compared to the control cells with basal levels of PKD1. We further investigated if the changes in glucose metabolism alter the enhanced tumorigenic characteristics of the cells on PKD1 overexpression. Our results demonstrate that the migration (Figure. 3-5A and 3-5B). and invasive potential (Figure. 3-6A, B) of PKD1 overexpressing cells can be reduced by inducing glucoprivic conditions using 2-deoxyglucose or potentiated by using lactate $(2 \mathrm{mM})$, an end product of aerobic glycolysis, in the cells. This further suggested the involvement of glucose accelerated mechanisms acquired by pancreatic cancer cells on PKD1 overexpression that accompanies enhanced tumorigenesis. 
A

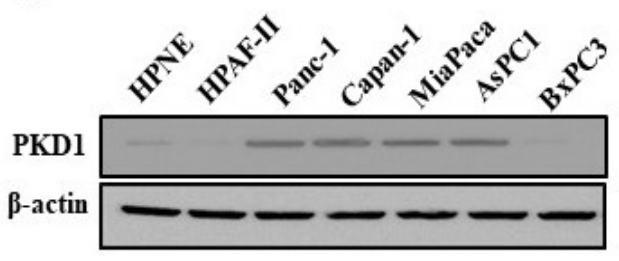

C

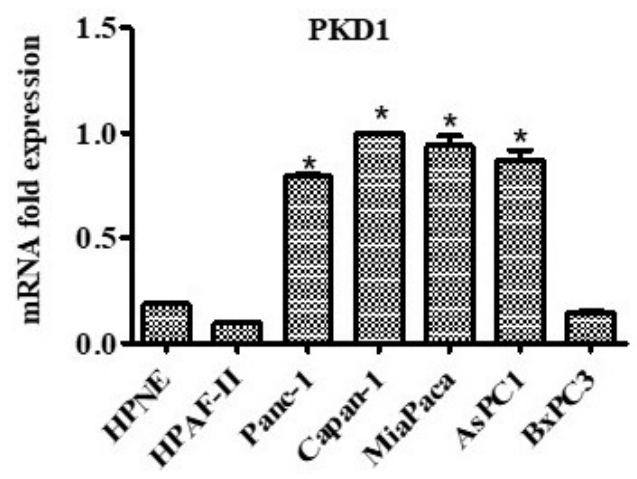

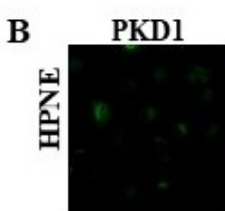
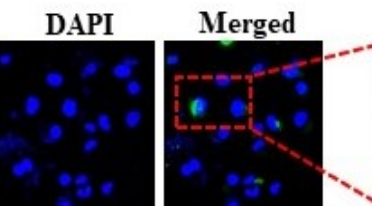

Zoomed
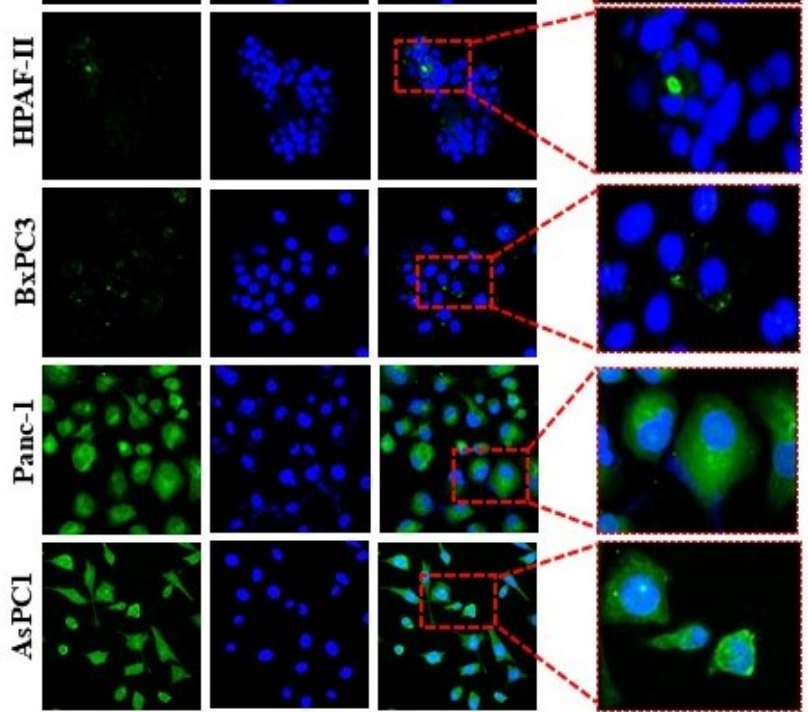

Figure 3-2. Differential Expression of PKD1 in Pancreatic Cancer Cells

(A) Western blot showing the expression level of PKD1 in different pancreatic cancer cell lines. HPNE is a normal pancreatic cell line. (B) Real-time PCR depicting the expression level of PKD1 in a panel of pancreatic cancer cells. (C) Confocal images depicting the expression level of PKD1 in pancreatic cancer cells. Cells were fixed, stained and analyzed by confocal microscopy. DAPI was used for nuclear staining. 
$\mathbf{A}$
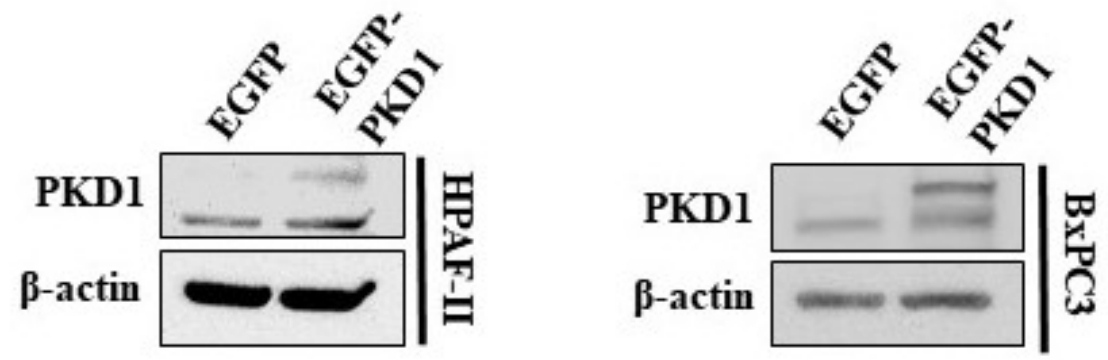

B
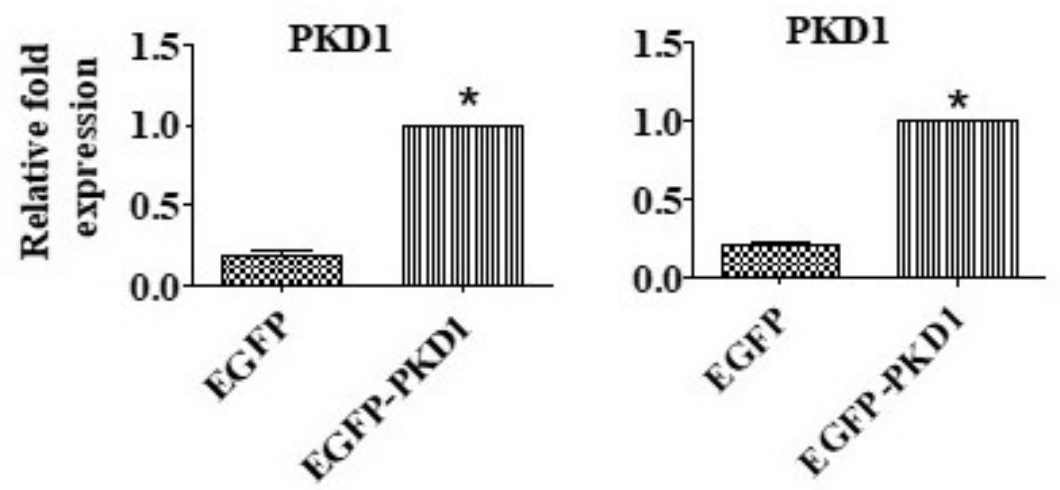

C
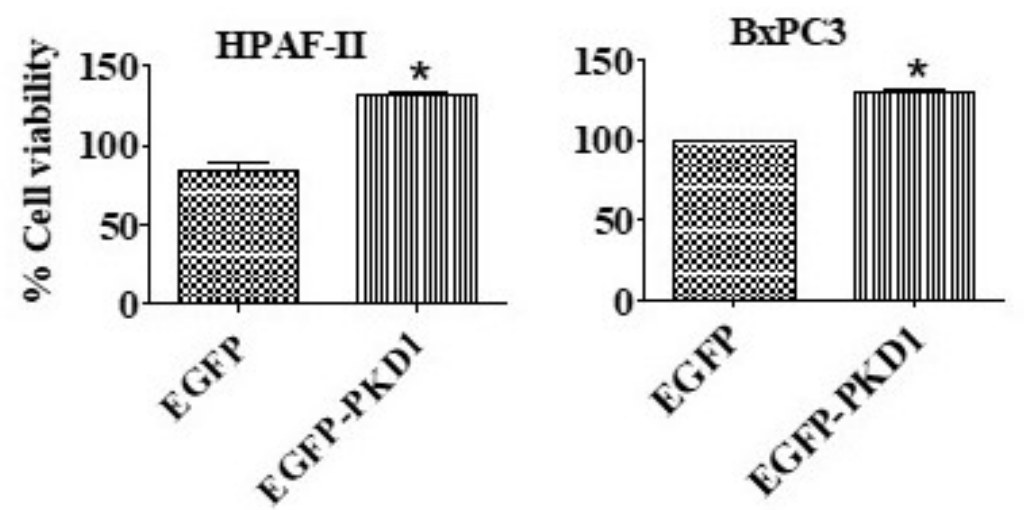

Figure 3-3. Overexpression of PKD1 Increases Cell Proliferation in Pancreatic Cancer Cells

(A) Pancreatic cancer cells (HPAF-II and BxPC3) were transfected with EGFP control and EGFP-PKD1 plasmid. Western blot showing the expression of PKD1. (B) Real-time PCR indicating the expression level of PKD1 after transfection. (C) Cell proliferation assay depicting the effect of PKD1 on cell viability. ${ }^{*} \mathrm{p}<0.05$. 
A
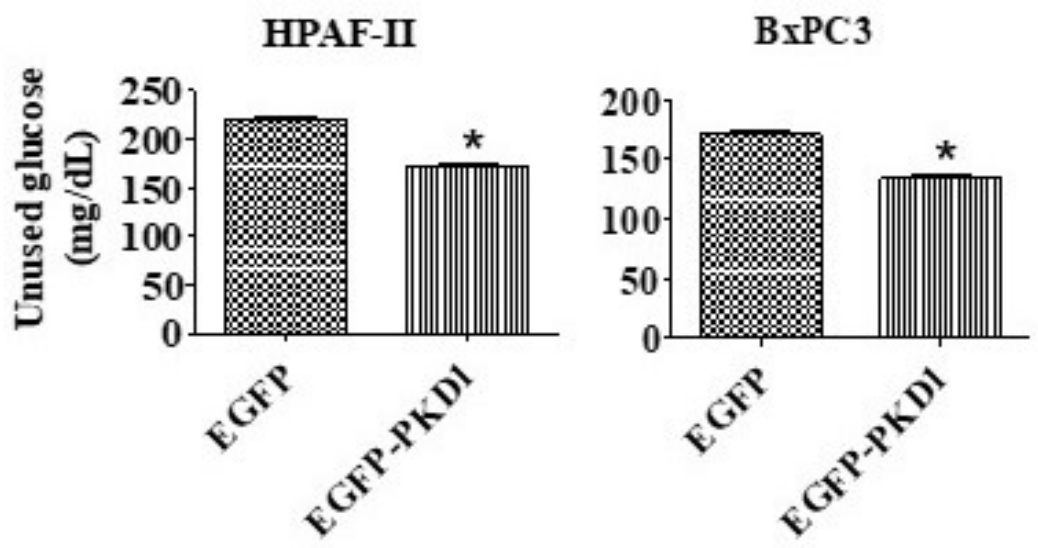

B
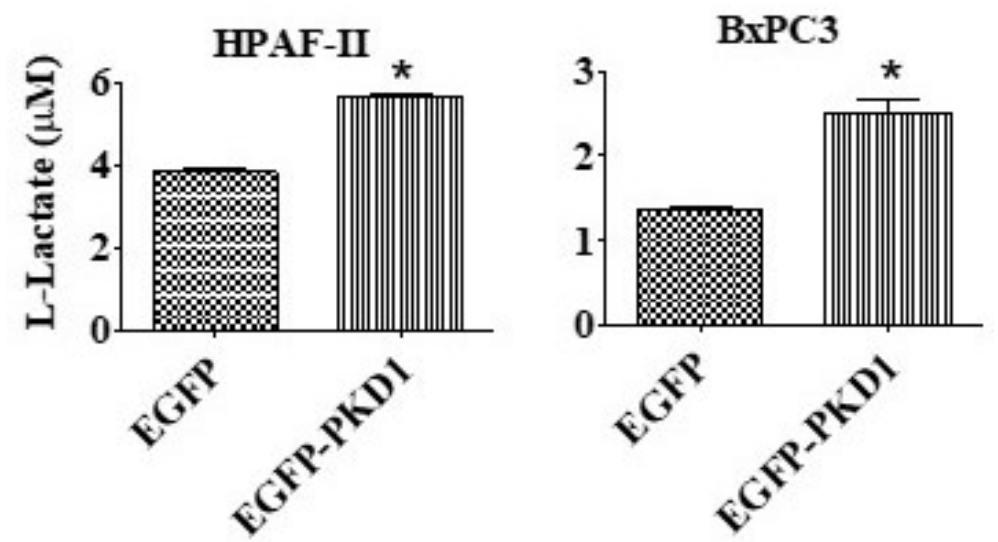

Figure 3-4. PKD1 Enhances Glucose Metabolism in Pancreatic Cancer

(A) Lactate and (B) Glucose assays were performed in HPAF-II and BxPC3 cells after PKD1 transfection. Media from cells was collected after 24 and 48 hours respectively, to determine glucose uptake levels and lactate production using commercial kits. ${ }^{*} p<0.05$. 
$\mathbf{A}$

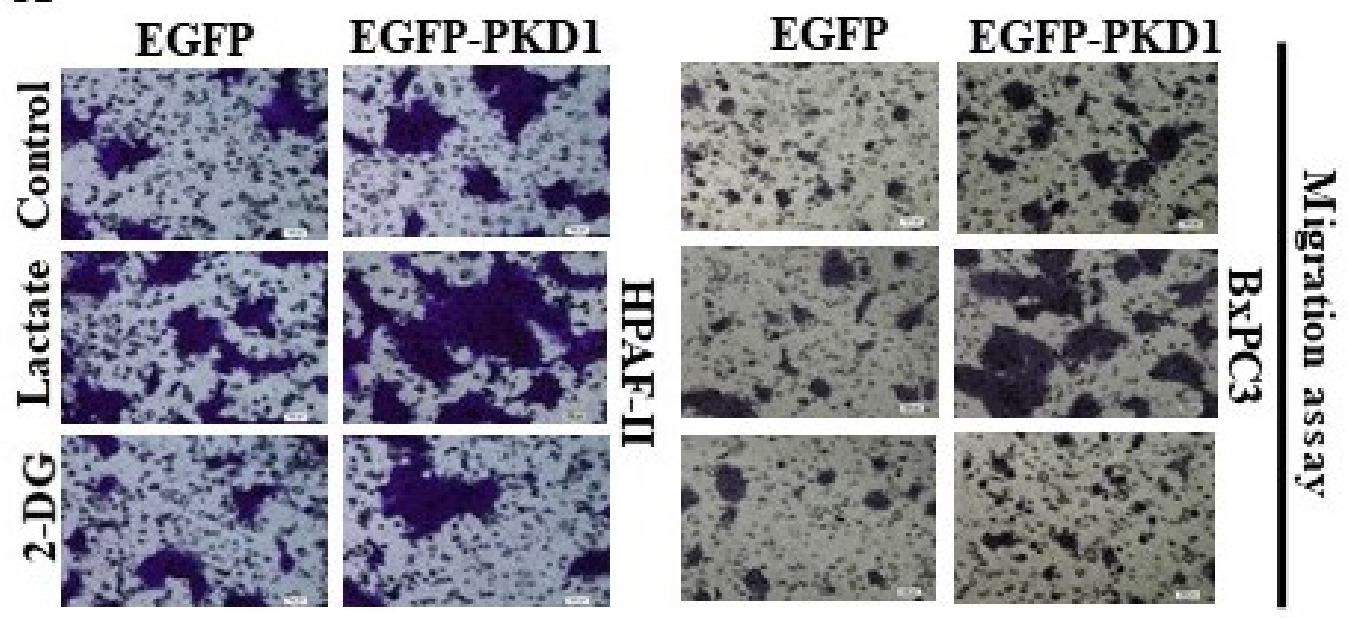

B

EGFP 血血 EGFP-PKD1
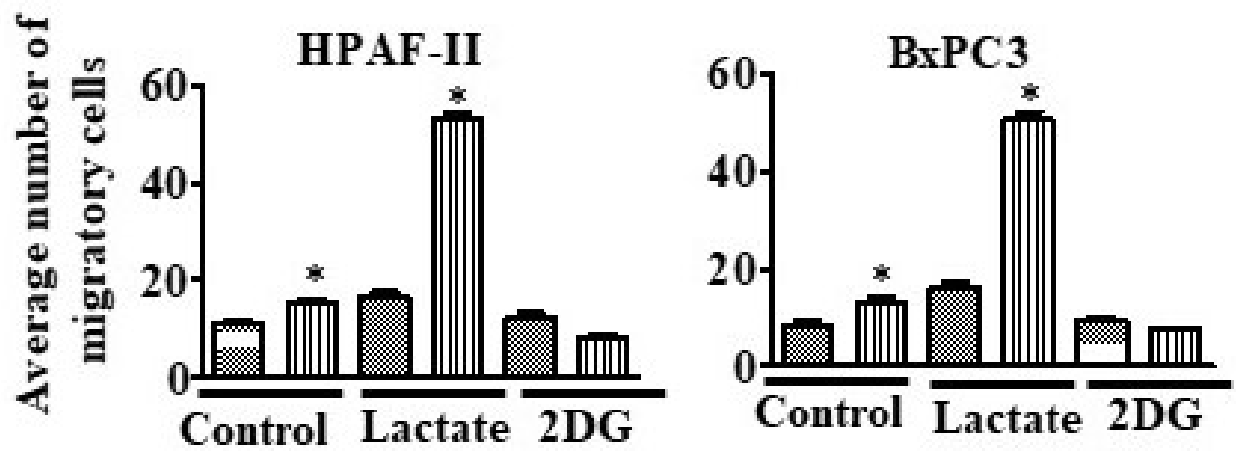

Figure 3-5. Glucose Metabolism is Enhanced by PKD1 in Pancreatic Cancer Cells (A) Boyden chamber migration assay was performed after treatment with L-Lactate $(2 \mu \mathrm{M})$ and 2-deoxyglucose $(2 \mathrm{DG}, 10 \mu \mathrm{M})$. The crystal violet stained cells were imaged at $18 \mathrm{~h}$. Magnification of images is 200X. (B) Quantitative depiction of total migratory cells. $\mathrm{n}=3 ;{ }^{*} \mathrm{p}<0.05$. 
A
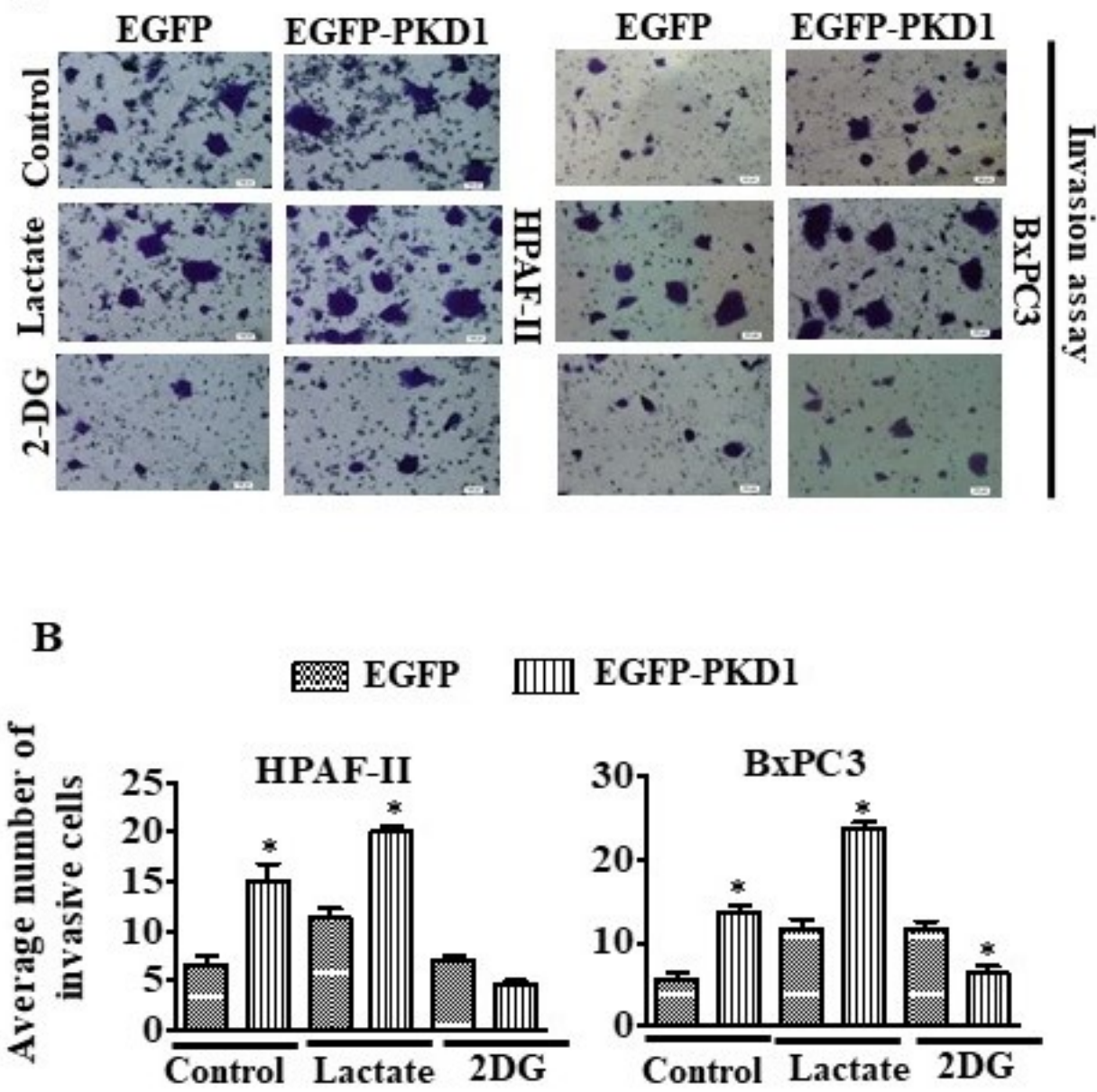

Figure 3-6. PKD1 Accelerates Glucose Metabolism in Pancreatic Cancer Cells (A) Matrigel invasion assay was conducted in pancreatic cancer cells after L-Lactate $(2 \mu \mathrm{M})$ and 2-deoxyglucose $(2 \mathrm{DG}, 10 \mu \mathrm{M})$ treatment. Staining of cells was done using crystal violet, and after 24 hours, the experiment was terminated and images were taken at 200X. (B) Representative quantification of the invasive cells. $n=3 ;{ }^{*} \mathrm{p}<0.05$. 


\section{PKD1 Expression Leads to Increased Expression of Signaling Proteins That Trigger Enhanced Glucose Metabolism}

Alteration of metabolic processes require the interplay between pletoire of signaling events involving changes in the expression of proteins. Glucose transporter 1 or GLUT1 is an important protein that facilitates the transport of glucose across the plasma membranes of cancer cells [173]. Our results demonstrate that PKD1 overexpression in PanCa cells, HPAF-II and BxPC3, results in an upregulation of protein as well as mRNA expression of GLUT1. We also observed an increased protein and mRNA expression of hypoxia-inducible factor or HIF-1 $\alpha$, which has a role in metabolic switching and regulates glucose metabolism [174] (Figure. 3-7A, B and (Figure. 3-8A, B). Therefore, we were interested to understand the underlying molecular mechanisms that may be involved in metabolic alterations accompanied by PKD1. Since mTOR signaling is widely reported to regulate HIF- $1 \alpha$ protein synthesis, we particularly investigated mTOR pathway[175]. mTOR is known as a master regulator of growth and metabolic state of cells in response to nutrients and extracellular stimuli. Interestingly, our results clearly demonstrated that PKD1 overexpression enhances the phosphorylation of mTOR (Figure. 3-7A, B). These results were confirmed when the silencing of endogenous PKD1 in Panc-1 cells (having high PKD1 expression) inhibited aforementioned events (Figure. 3-7C). In addition to the phosphorylation of mTOR, endogenous expression of GLUT-1 and HIF-1 $\alpha$ was observed to be reduced on silencing endogenous PKD1 expression, which suggests that PKD1 induced metabolic changes may be influenced by mTOR phosphorylation (Figure. 3-7C). We next sought to investigate whether the hypoxic environment has any effect on PKD1 induced tumorigenic effects. BxPC3 and HPAF-II cells cultured in a hypoxic environment displayed more cell proliferation (Figure. 3-9A, B) and high clonogenicity (Figure. 3-10, B) with formation of greater number of colonies on PKD1 overexpression as compared with cells under normoxic environment.

\section{PKD1 Increases Glucose Metabolism via mTOR C1 Activation}

We set out to analyze whether mTOR phosphorylation regulates the formation of either mTORC1 or mTORC2. mTORC1 is known to modulate HIF- $1 \alpha$, which stimulates glycolysis and glucose uptake. In order to elucidate the possible involvement of mTORC1, the major downstream effectors of MTORC1 are the ribosomal S6 kinase (S6K) and the inhibitory eIF4E-binding proteins (4E-BPs). mTORC1 activation phosphorylates $\mathrm{S} 6 \mathrm{~K}$ and 4E-BP, which phosphorylates/activates downstream proteins involved in initiation and elongation [176]. We found that PKD1 overexpression in PanCa cells results in enhanced phosphorylation of both the effectors of mTORC1, pS6kinase and 4EBP1. To our expectation, the silencing of endogenous PKD1 inhibited phosphorylation/activation of both the downstream effectors of mTORC1. In addition to pS6kinase and 4EBP1 upregulation, an upregulation of p-AKT, a major effector of mTORC2 was observed on PKD1 overexpression (Figure. 3-11A) that annotated the activation of both mTORC1 and mTORC2 that belongs to mTOR complex. Since 
A Cells: HPAF-II

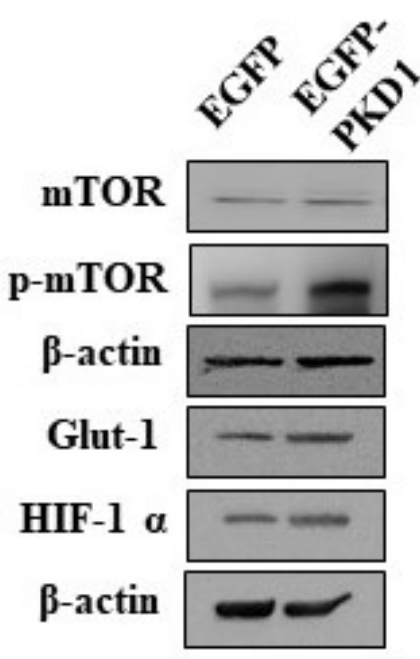

B Cells: $\mathrm{BxPC3}$

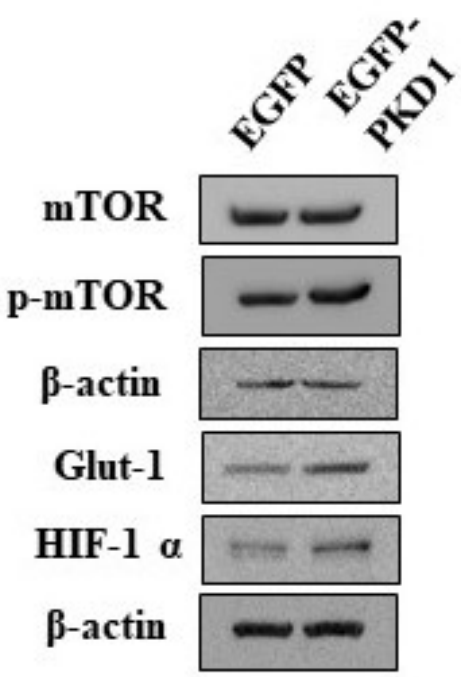

C Cells: Panc-1

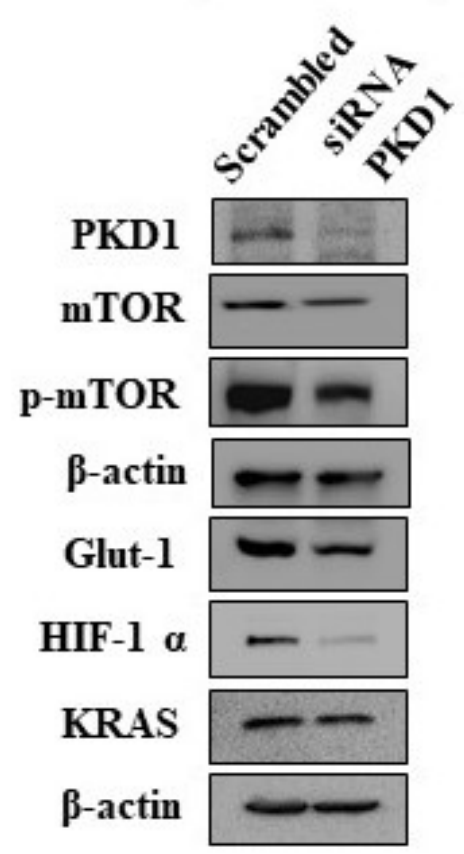

Figure 3-7. PKD1 Modulates the Expression of Important Proteins Involved in Enhanced Cancer Cell Metabolism

(A), (B) Western blot showing the expression level of key proteins associated with glucose metabolism after overexpressing PKD1, and (C) silencing PKD1. 
A

Cells: HPAF-II
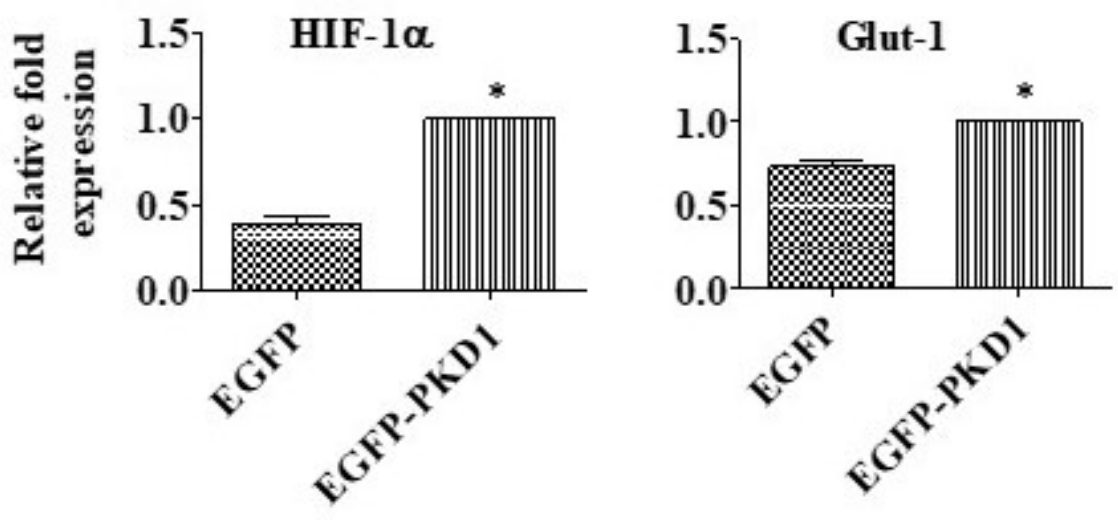

B

Cells: BxPC3
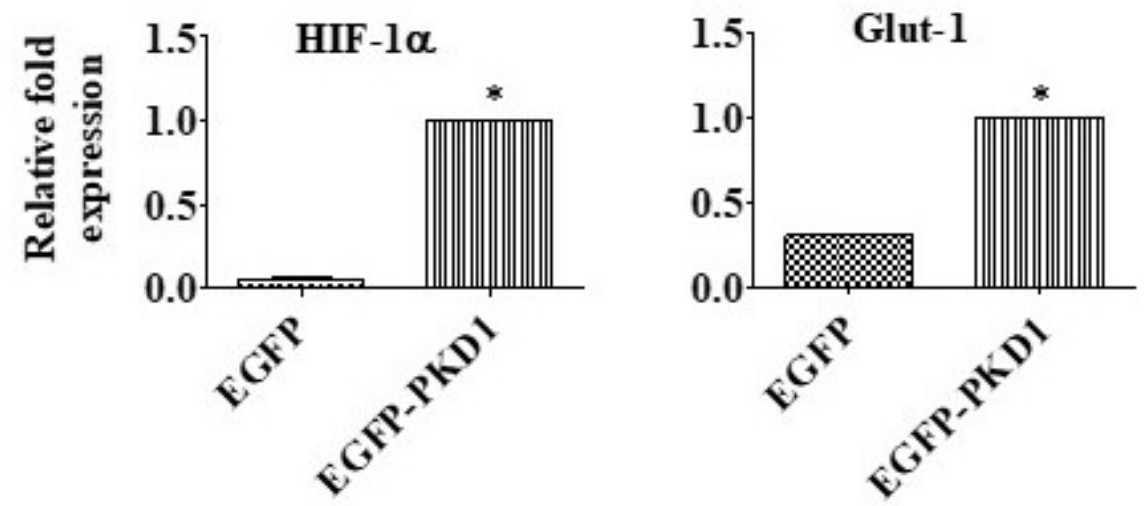

Figure 3-8. PKD1 Elevates the Expression of Associated Genes with Glucose Metabolism

(A), (B) Changes in real time expression of important genes involved in glucose metabolism by quantitative real-time PCR. Increased level of HIF-1 $\alpha$ and Glut-1

expression was observed in PKD1 expressing cells (HPAF-II and BxPC3 cells). $\mathrm{n}=3$; ${ }^{*} \mathrm{p}<0.05$. 
A

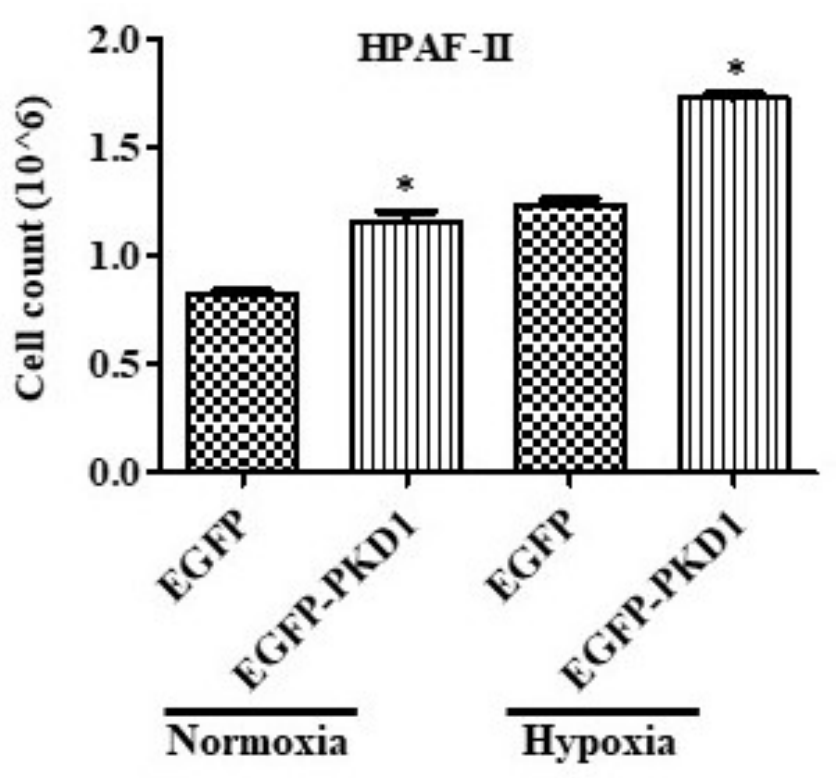

B

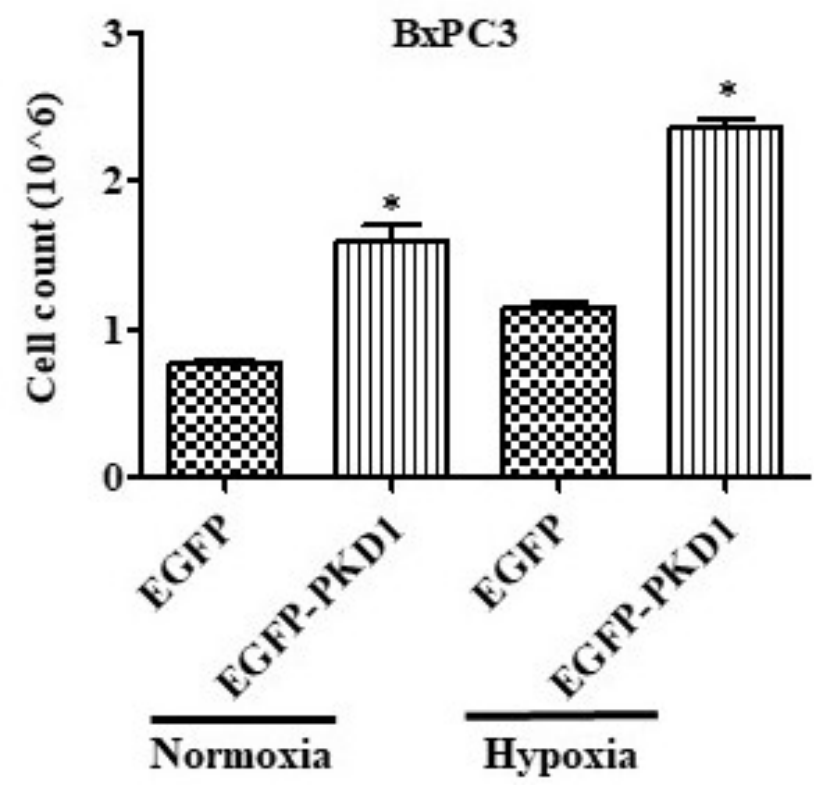

Figure 3-9. PKD1 Overexpression Increases Cell Proliferation Under Both Normoxic and Hypoxic Conditions

Effect of PKD1 on the pancreatic cancer cells was observed in normoxic and hypoxic environment. The cells were counted and recorded in (A) HPAF-II, and (B) BxPC3 cells. $* \mathrm{p}<0.05$. 

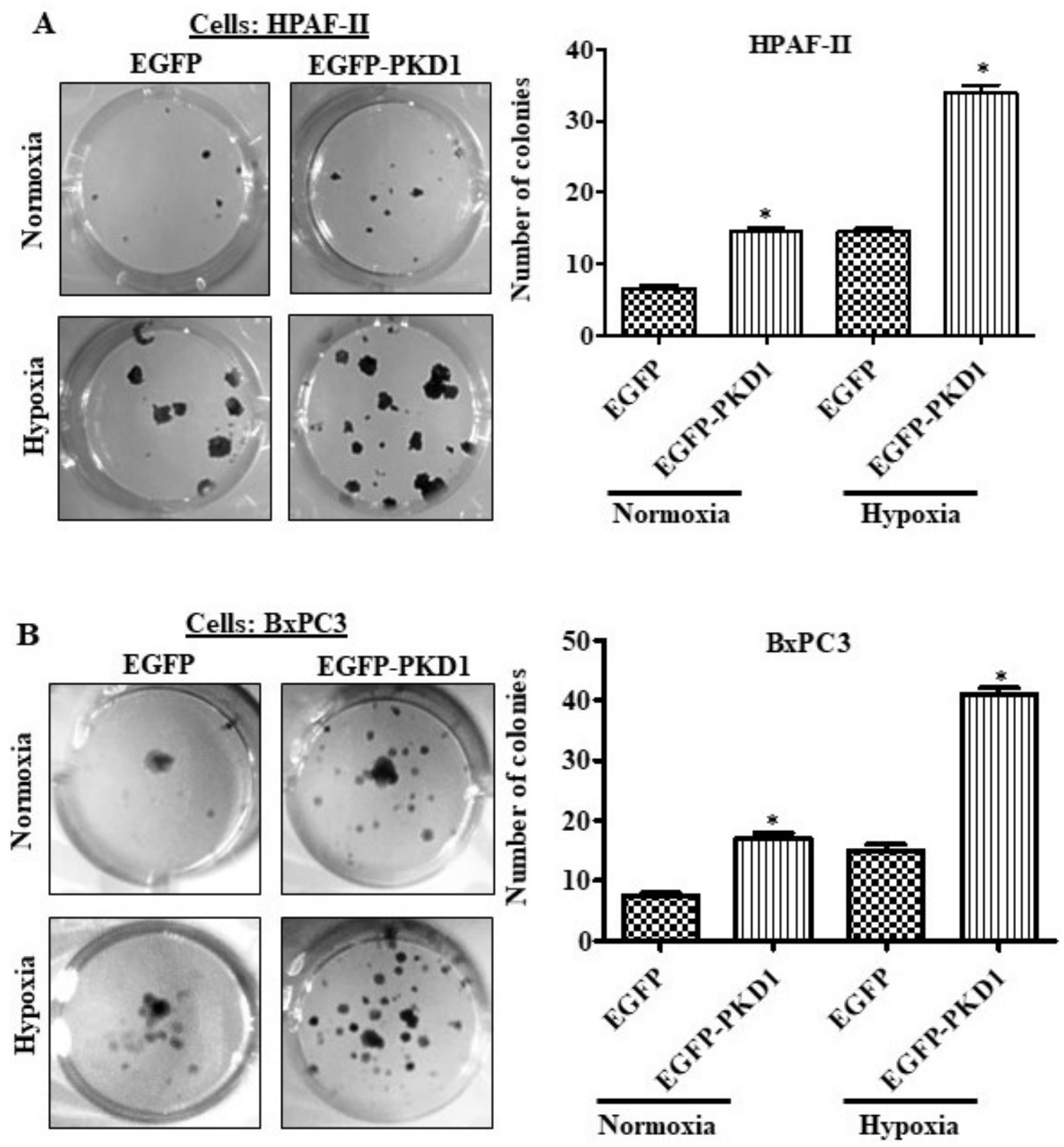

Figure 3-10. PKD1 Induced Cell Proliferation is Independent of Oxygen Tension Colony formation assay was performed under normoxic and hypoxic conditions in (A) HPAF-II and (B) BxPC 3 cells. 250 cells were seeded in 12 wells plate. The cells after 7 days were fixed using ice cold methanol, stained and imaged. $\mathrm{n}=3,{ }^{*} \mathrm{p}<0.05$. 

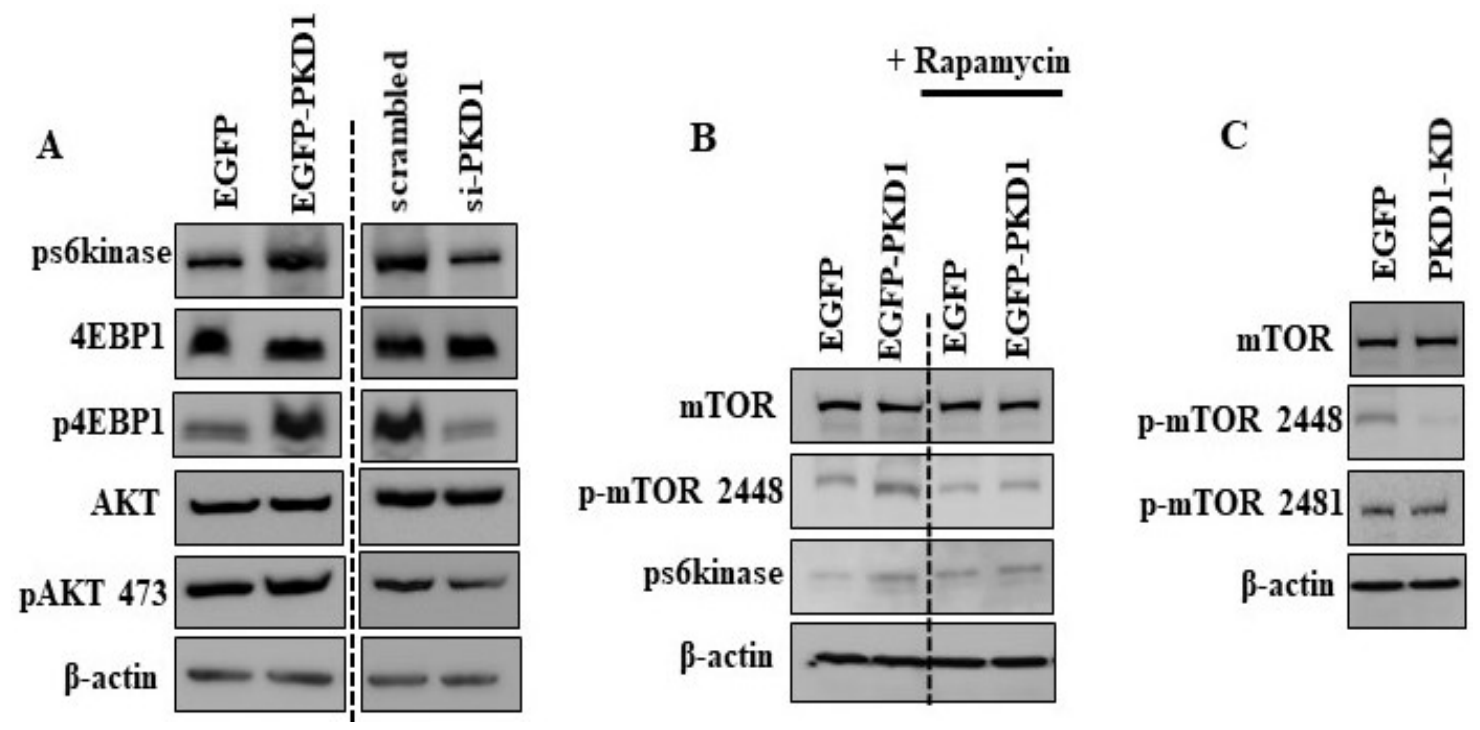

Figure 3-11. PKD1 Enhances Glucose Metabolism Through Activation of AKTmTOR Pathway

(A) Western blot to analyze the expression level of important proteins associated with mTORC1 and mTORC2 pathway. (B) Western blot depicting the expression of p-mTOR and ps6kinase in presence and absence of rapamycin. (C) Immunoblotting showing the expression of mTORC1 and mTORC2 after transfection with PKD1 kinase dead domain. 
mTORC1 is rapamycin sensitive, we investigated the effect of PKD1 overexpression in presence of Rapamycin on the phosphorylation of pS6kinase and mTOR-S2448, major phosphorylating domain of mTORC1. We observed that Rapamycin treatment prevented the increase in phosphorylation of both pS6kinase and mTOR-S2448, indicating that mTORC1 is the main effector of PKD1 (Figure. 3-11B). It is known that the kinase domain of PKD1 is involved in the activation/phosphorylation of proteins [86].

Therefore, in order to investigate whether the kinase region is responsible for the increased activity/phosphorylation of mTOR, BxPC3 cells were transfected with the PKD1 kinase dead domain (PKD1-KD). Immunoblotting demonstrated inhibition in the phosphorylation of mTOR-S2448 but not mTOR-S2481 (major phosphorylating domain of mTORC2) in cells transfected with PKD1-KD. The above results inferred two vital conclusions; PKD1 directly phosphorylates mTOR-S2448 through its kinase domain and mTORC1 being the downstream effector of PKD1 (Figure. 3-11C). We next asked whether mTORC1 is involved in PKD1 modulated glucose metabolism. For this, we investigated the changes in the lactate production and glucose consumption in PKD1 overexpressed cells in presence or absence of Rapamycin. Our results clearly showed that the PKD1 expressing cells in presence of Rapamycin failed to increase lactate production and glucose consumption (Figure. 3-12A). These effects were confirmed by using siRNA mediated knockdown of Raptor or Rictor, which are the determinants of the activation of mTORC1 and mTORC2, respectively [72], in addition to PKD1 overexpression in cells. siRNA directed to Raptor but not Rictor, inhibited the lactate production and glucose consumption triggered by PKD1. No noticeable changes were observed on silencing rictor. As raptor is an important component of mTORC1 complex [72], these results indicated an obvious role of mTORC1 pathway in the aberrant metabolism induced by PKD1 (Figure. 3-12B).

\section{PKD1 Expression Leads to Gemcitabine Resistance and Its Inhibition Sensitizes Gemcitabine}

High glucose accelerates cell proliferation in pancreatic cancer cells and imparts chemoresistance. Metabolic dysregulation is one of the most common and recognizable features of cancer. This facilitates the change in response to substrate availability due to metabolic demands of cell during proliferation, growth and cell survival [11].

Cancer/proliferating cells alter their ability to metabolize biomolecules to meet increased energy demands. It has been recognized that cancer cells increase glycolytic lactate production independent of oxygen availability [177].

In addition to studies related to the role of PKD1 in glucose metabolism, we were interested to investigate its effect on anticancer therapy. Therefore, we wanted to investigate the effect of aberrant glucose metabolism on the outcome of drug treatment in PanCa cells and investigate whether response to drugs can be affected by excessive glucose metabolism. Therefore, upon treatment of cells with lactate ( $2 \mu \mathrm{M}, 48$ hours), we observed an increase in the viability of the cells and resistance to gemcitabine treatment 
A

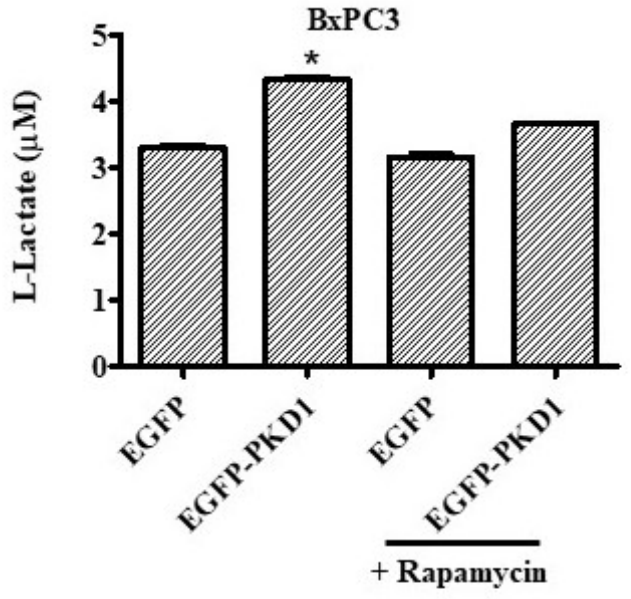

B

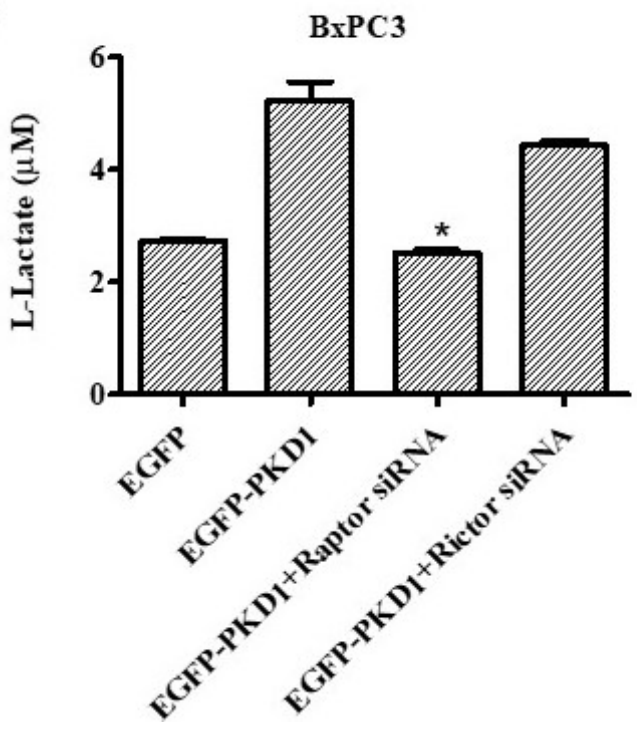

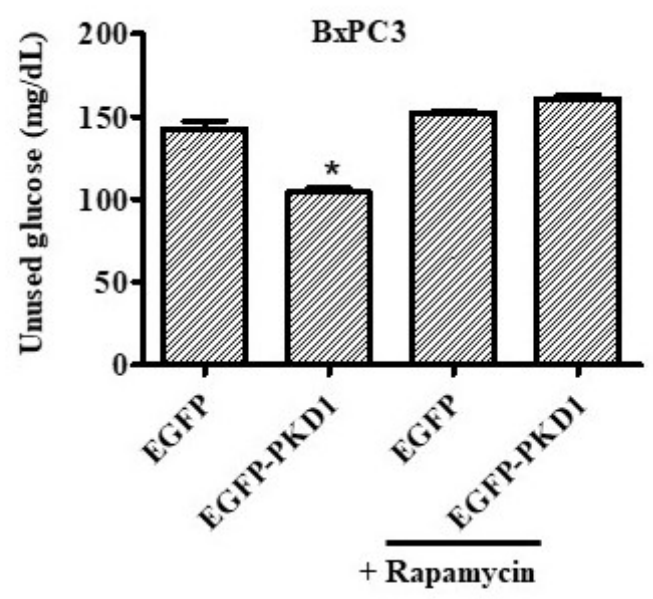

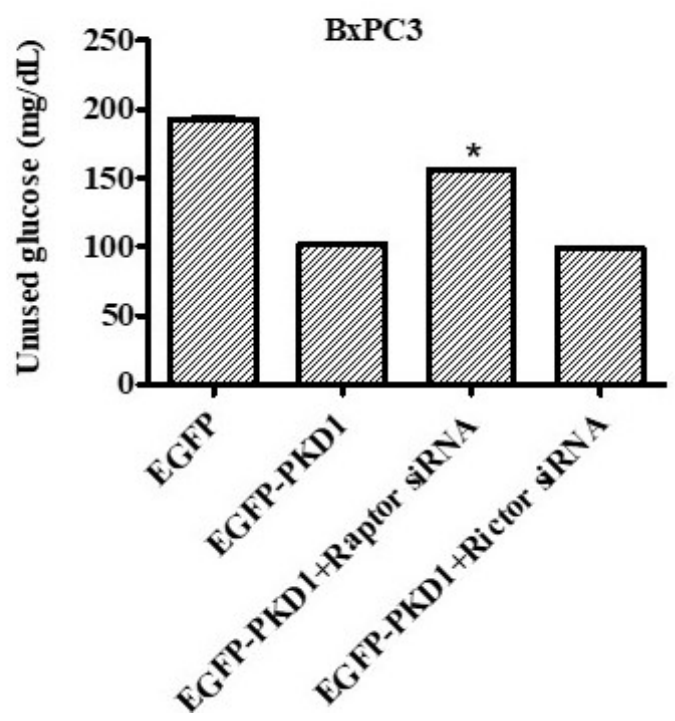

Figure 3-12. Glucose Metabolism is Accelerated by PKD1 Through mTOR Pathway

(A) Lactate and glucose assay in presence and absence of rapamycin (B) Lactate and glucose assays were performed after raptor and rictor silencing, which are essential components of mTORC1 and mTORC2 complex, respectively. 
(Figure. 3-13A). Treatment of cells with $2 \mathrm{DG}$ resulted in decreased viability and restored gemcitabine sensitivity (Figure.3-13B). Additionally, an induction of apoptosis by gemcitabine was observed in presence of $2 \mathrm{DG}$ as depicted by activation of apoptotic protein, Bax, cleavage of PARP and inhibition of antiapoptotic Bcl-2 (Figure.3-13C). In order to investigate the role of PKD1 in chemoresistance, we analyzed the effect of PKD1 overexpression on cell viability and gemcitabine resistance, we observed an enhanced cell viability on PKD1 overexpression (Figure.3-13Di) and resistance to gemcitabine (Figure.3-13Dii). Suppression of PKD1, using siRNA mediated knockdown, reduced the cell viability as well as gemcitabine (Figure.3-13Ei, ii) in the proliferation. These outcomes indicate that expression of PKD1 in pancreatic cancer cells result in chemoresistance, which attributes to the deregulated glucose metabolism and its suppression sensitizes gemcitabine in pancreatic cancer cells.

\section{Discussion}

Excessive glucose metabolism is a significant characteristic feature of cancer. Pancreatic cancer cells require large amounts of glucose for their survival. It leads to the fast growth and proliferation of the cancer cells. Many signaling molecules are reported to play an essential role in the aberrant metabolic processes, but further studies are still required to develop effective treatment strategies targeting glucose metabolism [157]. Several reports indicate an oncogenic role of the PKD1 protein in pancreatic cancer, but the mechanisms involved have not been sufficiently defined [87, 164]. This study demonstrates the role of PKD1 in enhancing glucose metabolism of pancreatic cancer cells and defines the underlying molecular mechanisms. Additionally, this study determines an important role of PKD1 in pancreatic cancer chemoresistance.

In the present study, we demonstrate that PKD1 is highly expressed in human Pancreatic ductal adenocarcinoma (PDAC) as compared with less or no expression in normal pancreatic tissues. This is a first study to our knowledge, which presents the expression profile of PKD1 in human PDAC. The oncogenic role of PKD1 in PanCa is growing interest among the scientific community and several reports suggest its role in pancreatic tumorigenesis $[87,89,164]$. Herein, we demonstrate a functional role of PKD1 in maintaining glucose metabolism of PanCa cells and illustrate signaling mechanisms that regulate the underlying molecular processes accompanying altered glucose metabolism. The results in this study demonstrate that PKD1 overexpression leads to enhanced lactate production and glucose consumption while PKD1 knockdown may lead to reduction in both. The results were similar with the supplementation of cells with lactate that potentiates the migration and invasion of PanCa cells. While supplementation of cells with 2-DG, a glucose analog that inhibits glycolysis [28, 38], restores the PKD1 induced tumorigenic effects. These data suggest that PKD1 promotes tumorigenesis by modulating glucose metabolism and warrants further investigation for underling mechanisms. As PKD1 was also observed to effect the expression hypoxiainducible factor 1 (HIF-1), further investigations revealed hypoxic environment being conducive to increased effects on cell growth, as depicted by ability of cells to colonize and warranted further investigation. 


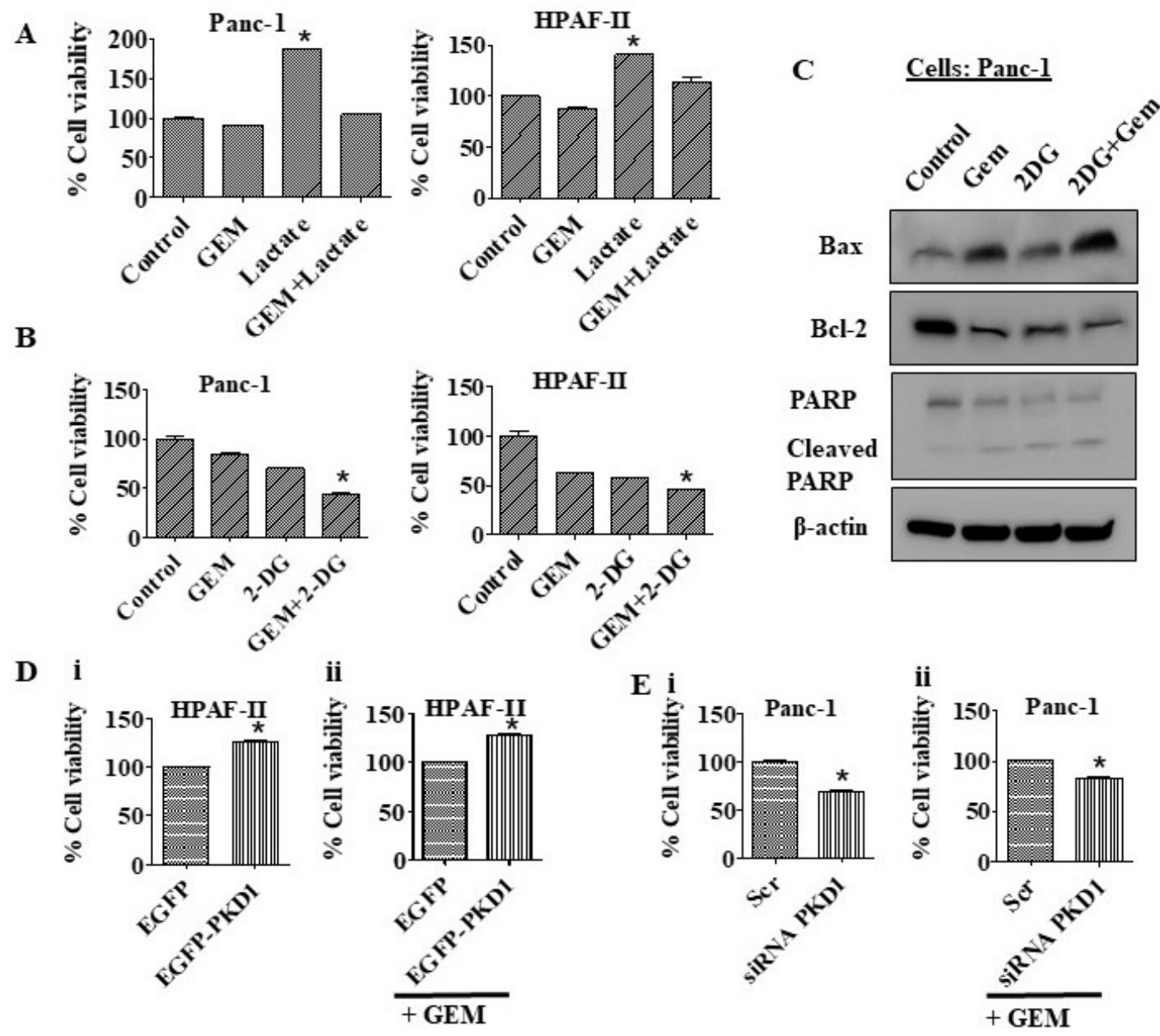

Figure 3-13. PKD1 Expression Leads to Chemoresistance and its Inhibition Sensitizes Gemcitabine

(A) Cell proliferation assay in pancreatic cancer cells in presence of L-Lactate and gemcitabine (B) Cell viability experiment in pancreatic cancer cells was performed in presence of 2-DG and gemcitabine (C) Western blot showing the expression of apoptotic proteins after treatment with 2DG and gemcitabine (D), (E) MTT assay in PKD1 expressing and null cells was conducted in presence and absence of gemcitabine. 
mTOR is the conserved serine/threonine kinase that regulates the proliferation and metabolism in cells. mTOR exists in two distinct complexes with different protein components and downstream substrates; mTOR complex C1 and C2 [178]. mTORC1 stimulates HIF-1 $\alpha$ regulating various enzymes for glucose metabolism, including glucose transporters [179]. This study demonstrates that PKD1 phosphorylates/activates mTORC1 in pancreatic cancer cells as was depicted by the increased activation/phosphorylation of its main effectors, pS6K and 4EBP1 on PKD1 overexpression. However, the kinase dead mutant of PKD1 did not enhance the phosphorylation/activation of the major phosphorylating domain of mTORC1, mTORS2448. The detection of reduction in the phosphorylation of mTOR-S2448 in cells expressing kinase mutant of PKD1 can be explained well with the fact of a new phosphorylated protein not being produced while the accumulated protein being degraded with time (Fig. 6C). These results indicate that PKD1 activity was necessary for mTORC1 activation. mTORC1 inactivation by rapamycin is critical for glucose metabolism [180, 181]. Rapamycin binds to the FK506-binding protein of $12 \mathrm{kDa}$ (FKBP12) and inhibits the activity of mTORC1 [182]. Our findings further demonstrate that $\mathrm{mTORC} 1$ activation by PKD1 regulates metabolic alterations in pancreatic cancer cells. These results were quite convincing as the treatment of rapamycin inhibited PKD1 accelerated lactate production as well as glucose consumption in cancer cells. Additionally, the significance of mTORC1 activation in PKD1 induced metabolic alterations was established on silencing raptor, the main determinants of the activation of mTORC1, which inhibited the metabolic changes associated with PKD1. Since no significant alterations were detected on silencing rictor, confirms the association of mTORC1 with PKD1 induced metabolic alterations. In other words, it can be stated that the promotion of glycolysis by PKD1 can be regulated by mTORC1 suppression and this concept can be utilized to develop novel therapeutic strategies.

Another major finding in this study demonstrates the role of PKD1 in chemoresistance. The results presented in this study suggesting that PKD1 induces chemoresistance due to aberrant glucose metabolism are significant. Additionally, we demonstrate that altered glucose metabolism interferes with the sensitivity of the drug and its outcomes in PanCa cells. Our data suggests that PanCa cells become resistant to chemotherapeutic drugs in presence of enhanced glucose, which can be overcome by depleting the glycolysis. An induction of apoptosis by gemcitabine was achieved in presence of 2DG as depicted by activation of apoptotic protein, Bax, cleavage of PARP and inhibition of anti-apoptotic Bcl-2. Our observations identify that modulation of PKD1 expression can turn the PanCa cells sensitive. This was indicated by using siRNA mediated knockdown of PKD1 that rendered these cells sensitive to gemcitabine treatment.

In conclusion, we present the expression profile of PKD1 in human PanCa tissues and demonstrate that PKD1 is aberrantly expressed in PanCa as compared with normal pancreatic tissues. Our results suggest a novel role of PKD1 contributing to aberrant glucose metabolism in pancreatic cancer cells which accelerates PanCa tumorigenesis and chemoresistance. These interesting findings strongly indicate that PKD1 can be 
explored as a novel therapeutic target and as a biomarker, inhibition of which can reduce pancreatic cancer progression and metastasis. The association of PKD1-induced mTOR pathway can be used as a therapeutic strategy for the emergence of new drugs to target pancreatic cancer. Moreover, as suggested by our results, PKD1 has a role in chemoresistance owing to dysregulated glucose metabolism in pancreatic cancer. Therefore, it can further be exploited to increase the survival rate of pancreatic cancer patients. The overall mechanism of PKD1-induced metabolic reprogramming through mTOR is depicted in Figure 3-14.

Further, we wanted to identify a drug which could inhibit the elevated glucose metabolism in pancreatic cancer with less toxicity to the normal cells. Also, it was essential to determine the therapeutic potential of the drug in terms of inhibition of the oncogenic protein molecules identified earlier in our studies (MUC13 and PKD1).

Therefore, we selected a plant-derived compound, steviol for our further studies, which could both restore glucose metabolism and act as a chemo-preventive agent. 


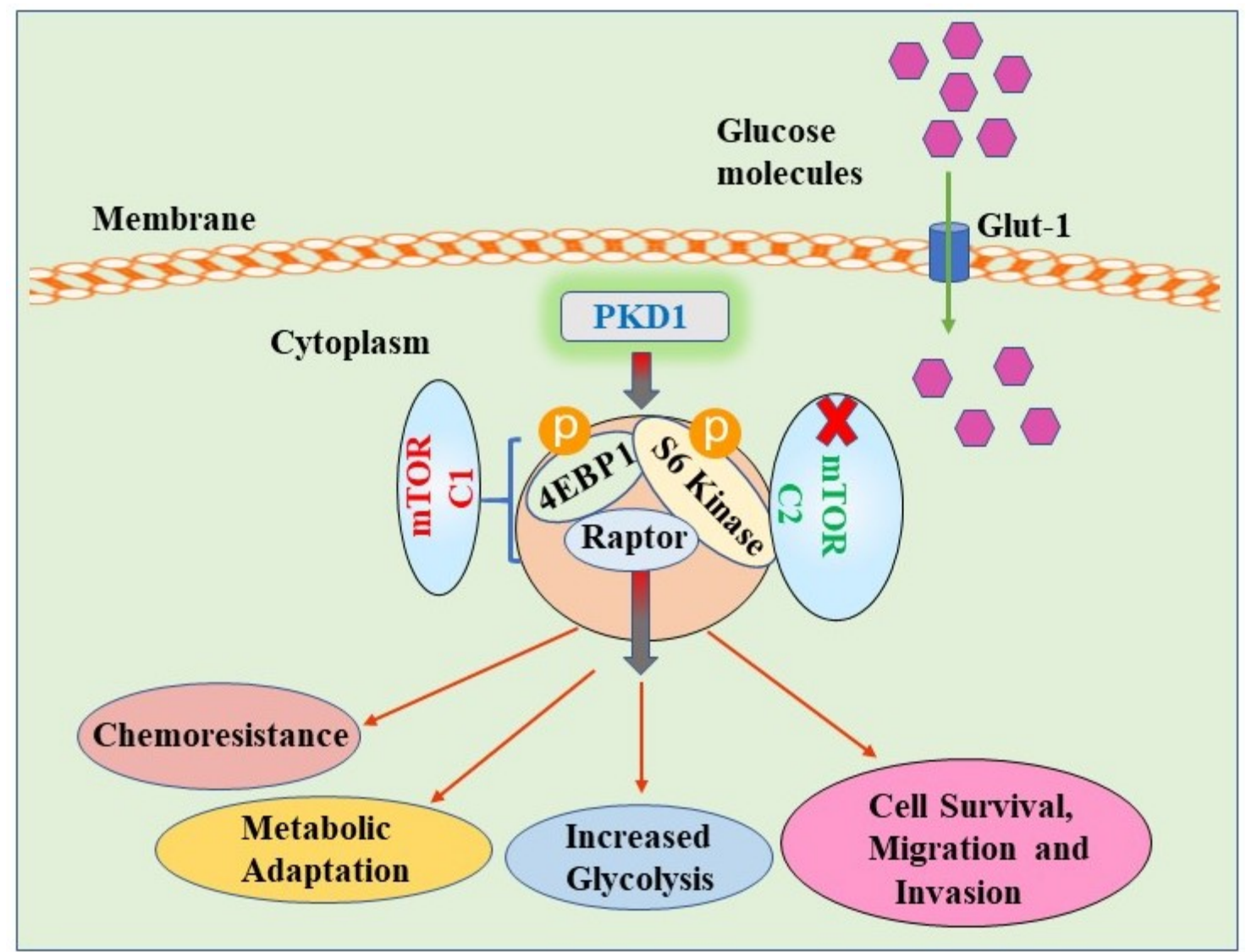

Figure 3-14. Proposed Mechanism Describing the Role of PKD1 in Impaired Glucose Metabolism in Pancreatic Cancer

PKD1 induces metabolic reprogramming in pancreatic cancer through mTOR pathway. This mechanism results in increased glucose metabolism, proliferation and chemoresistance of pancreatic cancer cells. 


\section{CHAPTER 4. STEVIOL: A NATURAL SWEETENER, INHIBITS PANCREATIC CANCER PROGRESSION THROUGH SUPPRESSION OF ABERRANT GLUCOSE METABOLISM}

\section{Introduction}

Pancreatic cancer (PanCa) is one of the deadliest malignancies, and accounts for many mortalities worldwide [1]. The lack of early detection, chemoresistance and metastatic profile contributes to the highly aggressive behavior of this disease [1]. Therefore, it is essential to understand the underlying mechanisms of this disease, which can prolong the survival rate of the affected patients.

Stevia is a plant product and is obtained from Stevia rebaudiana. It has a low calorie content, but is almost 300 times sweeter than the normal cane sugar. The metabolism of steviol begins in the human gut when stevioside (precursor of steviol) is converted to steviol 16,17 $\alpha$-epoxide, and then to steviol through the action of bacteria present in the gut [130]. After the completion of the metabolic process, it is relatively easier for steviol to get absorbed in the blood following oral administration. New studies have indicated that stevioside is very effective in reducing high glucose levels and inflammation. Reports also suggest that it has potent anti-cancer properties in different cancers, demonstrating its therapeutic properties [126-129]. Stevioside has been found to be a potent compound, as it can decrease the glucose levels in blood in humans [131] and, also in rats [132]. These indicate the safety, low toxicity, and valuable properties of steviol on glucose metabolism. Nevertheless, the role of steviol in the regulation of glucose metabolism has not been clearly elucidated.

Aberrations in the translation process is a unique feature associated with cancer progression. A variety of key tumor suppressor and oncogenic factors are involved in this process and drive this mechanism [79]. The initiation step of translation process is the most prominent and tightly co-ordinated phase. Deregulation of some of the important pathways- PI3K/AKT/mTOR are mainly responsible for the abnormal functioning of the translation initiation apparatus $[183,184]$. The eukaryotic initiation factors (eIFs) are the key proteins which are strongly associated with the translation complex. So far, 6 eIFs have been reported, namely, eIFs1-6. Alterations in their regular function leads to emergence of an oncogenic behavior [79, 184]. Therefore, targeting these molecules is essential for cancer treatment, paving the way for development of new therapeutic drugs.

The cancer cells undergo dysregulated glucose metabolism in order to sustain growth and proliferation rapidly. This also involves the upregulation of tumor promoting genes [185]. In comparison to the quiescent cells, the cancerous cells do not use oxidative phosphorylation, but rather utilize the aerobic glycolysis mechanism [186]. This mechanism is also called the Warburg effect and is extensively researched. During this process, large amount of energy is released in the form of ATP as glucose uptake is high in comparison to the normal cells, which in turn also secretes abundant amount of lactate. 
This also creates an acidic microenvironment [187]. In addition, the chemo-resistant tumor cells need more glucose for their survival and continuous growth. Hence, it is mandatory to target the glucose metabolic networks and increase sensitivity of the drug resistant cells, in order to enhance the efficiency of the drugs used for chemotherapy, as a result also increasing the lifespan of the suffering patients due to pancreatic cancer [188].

As steviol modulated glucose metabolism in our current study, we believe that this might be one of the reasons for its mode of action in influencing translation apparatus. This is the first study on steviol describing the underlying mechanism through inhibition of translation initiation in pancreatic cancer. In addition, we also observed inhibitory role of steviol as it suppressed the expression of both MUC13 and PKD1 (proteins in association with glucose metabolism), in our studies. Overall, this research work identifies the potential role of steviol in inhibiting pancreatic cancer through various mechanisms, and suggests that it can be a promising drug candidate for therapeutic intervention either alone or with combinatorial strategies.

\section{Materials and Methods}

\section{Cell Culture}

Pancreatic cancer cells (HPAF-II and AsPC1) were used for this study. These cells were maintained in DMEM/F12 or RPMI media, respectively, containing $10 \%$ fetal bovine serum. The incubation conditions used were $37^{\circ} \mathrm{C}$ temperature with $5 \% \mathrm{CO}_{2}$.

\section{Western Blotting}

Cell lysates were prepared and western blotting was performed [11]. Samples were loaded on a gel (4-20\%), ran for 90 minutes. Thereafter, the gel was transferred onto a PVDF membrane for 70 minutes duration. Further, the membranes were blocked in 5\% milk for 1 hour, on a slow shaker, followed by incubation with the respective primary antibodies overnight at $4^{\circ} \mathrm{C}$. The secondary antibodies were added for 1 hour next day, after washing the membranes with $1 \mathrm{X}$ TBST buffer, and the blots were developed with an ECL solution on a UVP machine.

\footnotetext{
Antibodies

Primary antibodies from Cell Signaling Technology were used to analyze the expression of important proteins, Bcl-2 (catalog \# 4223S), Bax (catalog \# 2772S), Bcl-xl (catalog \# 2764S), eIF4e (catalog \# 9742S), peIF4e (catalog \# 9741S), 4EBP1 (catalog \# 9452S), p4EBP1 (catalog \# 2855S), mTOR (catalog \# 4517S), p-mTOR (catalog \# 5536S), eIF4B (catalog \# 3592S), peIF4B (catalog \# 3591S), eIF4G (catalog \# 2498S), peIF4G (catalog \# 2441S). Cleaved PARP (sc-8007) and Cyclin D1 (sc-753)
} 
antibodies were obtained from Santa Cruz Biotechnology. Beta-actin antibody was obtained from Sigma (catalog \# A2228). The secondary antibodies from Promega were used for rabbit (catalog \# 4011), and mouse (catalog \# 4021) conjugated with horseradish peroxidase.

\section{Proliferation Assay}

MTT assay was performed on pancreatic cancer cells to access the cell proliferation properties. This assay was terminated after $48 \mathrm{hrs}$ by adding $20 \mu \mathrm{l}$ of MTT reagent to the cells. After 2 hours of incubation, $100 \mu 1$ of DMSO was added to the cells, and the plate was kept on shaker for 10 minutes. Further, the absorbance was recorded at $570 \mathrm{~nm}$, the viability of cells was determined and quantified.

\section{Migration Assay}

Cell migration assay was performed using Boyden chamber $[169,170] .5^{*} 10^{\wedge} 4$ cells were seeded per well in a 96 well plate on the upper chamber with media devoid of serum. On the other hand, the lower chamber contained $250 \mu 1$ of media with serum. The cells were treated with steviol at different concentrations and further incubated for 18 hours. The cells were fixed with 4\% PFA, followed by staining using crystal violet reagent for about 1 hour. Thereafter, the plate was washed using tap water. The images were captured after the cells were dried.

\section{Invasion Assay}

The cell invasion assay was conducted in pancreatic cancer cells using Matrigel Invasion Chambers obtained from BD Biosciences [149]. The chambers were rehydrated for 2 hours in media without any serum content. Further, the cells were seeded $\left(25^{*} 10^{\wedge} 4\right.$ /chamber) in $500 \mu \mathrm{l}$ of serum-free media. Next day, the cells were treated with steviol at various concentrations in the chamber followed by incubation of 24 hours. Fixation of cells was done using cold methanol and staining was performed using crystal violet. Then, the washing of cells was done, and images were captured after 24 hours.

\section{Real-Time xCELLigence Assays}

To analyze the effect of steviol on pancreatic cancer cells, real-time proliferation and migration assays were conducted on xCELLigence instrument. Cells were seeded on the respective plates and were incubated in a $37^{\circ} \mathrm{C}$ incubator with $5 \% \mathrm{CO}_{2}$ condition. The experiment was terminated at the indicated time points and the results were analyzed. 


\section{Clonogenic Assay}

Cells were seeded (250) in 12 wells plate, and were incubated for 3-4 days to form colonies. The treatment was done immediately as soon as colonies started to appear. The experiment was terminated following 15 days after washing cells with cold PBS, fixed with cold methanol, and cells were stained with crystal violet. Further, the plates were left for drying and then the images were captured. The number of colonies were recorded and compared.

\section{Cell Cycle Studies}

This assay was performed through the Telford method. The cells were seeded $\left(0.5^{*} 10^{\wedge} 6\right)$ in a 6 -wells plate, and was incubated overnight. Pancreatic cancer cells were treated with steviol for 12 hours and 24 hours. The cells were trypsinized, washed with cold PBS and fixed with $70 \%$ ethanol. Further, the cells were incubated at $4^{\circ} \mathrm{C}$ for 1 hour and later stained with propidium iodide for about 4 hours in the dark (at $4^{\circ} \mathrm{C}$ ). The analysis was done in an FL2 channel, using cell cycle instrument, and ModFit software.

\section{Lactate Assay/ Glucose Assay}

Both assays were conducted as described in our previous publication [11]. The assays were conducted following the instructions mentioned in the kits (Cayman Chemicals). Thereafter, the absorbance readings were recorded, followed by plotting of the respective graphs based on the calculations.

\section{Statistical Analysis}

The statistical analysis for this manuscript was conducted by Student's t test. The p-values $<0.05$ were accounted as statistically significant.

\section{Results}

\section{Steviol Effectively Suppressed MUC13 and PKD1 Proteins}

We wanted to first investigate the therapeutic potential of steviol with respect to the proteins identified to be involved in dysregulated glucose metabolism, in our previous chapters. Therefore, we accessed the inhibition of the oncogenic proteins, MUC13 and PKD1, with steviol treatment. The concentration of steviol selected was based on some preliminary studies in our lab. It was observed that the expression levels of both MUC13 and PKD1 proteins were significantly decreased in a dose-dependent manner (Figure 
4-1A, B). This exciting data indicates that steviol, as a drug has a lot of therapeutic potential as it can significantly inhibit these upregulated oncogenic molecules, and therefore should be explored further to extend the survival chances of pancreatic cancer patients.

\section{Steviol Inhibits Cell Proliferation and Colony Formation of PDAC Cells}

In order to investigate the anti-tumorigenic properties of steviol, functional assays were performed in pancreatic cancer cells. Steviol treatment showed significant inhibition of cell proliferation in a dose-dependent manner. It demonstrated an IC 50 of $10 \mathrm{mM}$ concentration. Similar trend was observed in both HPAF-II and AsPC1 cell lines (Figure 4-2A).

Also, the colony forming abilities of pancreatic cancer cells (AsPC1 and HPAF-II cells) were remarkably suppressed by steviol as compared to the untreated cells (Figure 4- 2B).

\section{Steviol Suppresses Cell Migration and Cell Invasion Capabilities of Pancreatic Cancer Cells}

It was interesting to evaluate the functional characteristics of steviol on PDAC cells. For this, pancreatic cancer cells were treated with steviol at various concentrations and it was observed that steviol efficiently inhibited the cell migration (Figure 4-3A) and cell invasion abilities (Figure 4-3B), in a concentration-dependent manner. This indicated the anti-cancer properties of steviol in pancreatic cancer. Also, the suppression of proliferation and migration in HPAF-II cells was evaluated by xCELLigence assay after treatment with steviol. This also demonstrated that steviol was very effective in substantially reducing the proliferative abilities and migratory potential of pancreatic cancer cells in a dose-dependent way (Figure 4-4).

\section{Steviol Suppresses Translation Initiation of Pancreatic Cancer}

Disruption in the translation initiation machinery directly relates to carcinogenesis. Various oncogenic molecules and tumor suppressors play a major role in this pathway[79]. Therefore, it was imperative to understand the mechanism of action of steviol with regard to this signaling pathway. The expression of proteins associated with translation was analyzed, after treatment of pancreatic cancer cells with steviol at different time points The translation machinery was significantly suppressed as depicted by the immunoblotting assay (Figure 4-5A, B). The most important associated proteins were significantly downregulated with treatment of steviol such as peIF4E, peIF4B, peIF4G, p4EBP1, and p-mTOR. These molecules are required for the proper functioning of the translation initiation process. Steviol treatment clearly inhibited the expression of these essential proteins, which in turn hampered the formation of new proteins. It has 
A

Cells: HPAE-II
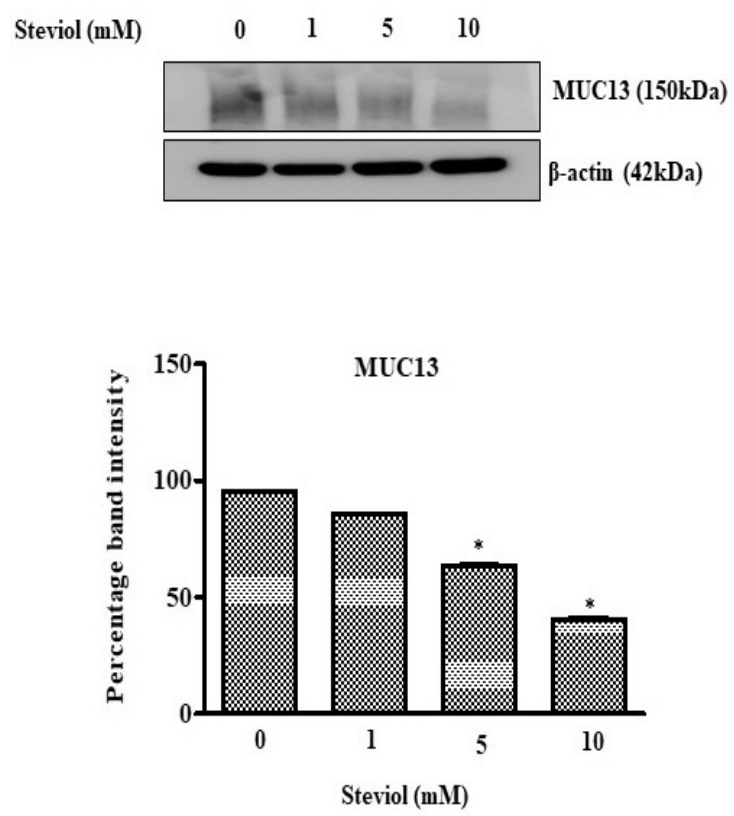

B

Cells: AsPCl
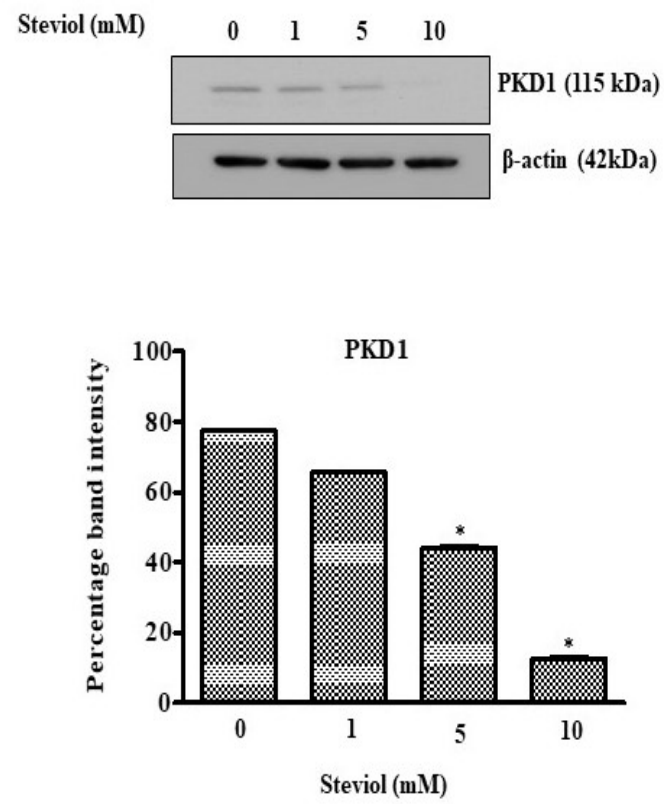

Figure 4-1. Steviol Inhibits the Expression of MUC13 and PKD1 Proteins (A) HPAF-II cells (having high MUC13 expression) were subjected to steviol treatment at the indicated concentrations for 24 hours. Whole cell lysates were prepared, and immunoblotting was done to determine the expression of MUC13 protein. (B) Similarly, AsPC1 cells, which are PKD1 expressing cells, were treated with steviol under similar conditions. The cellular lysates were made and used for western blotting to analyze the PKD1 expression after steviol treatment. 

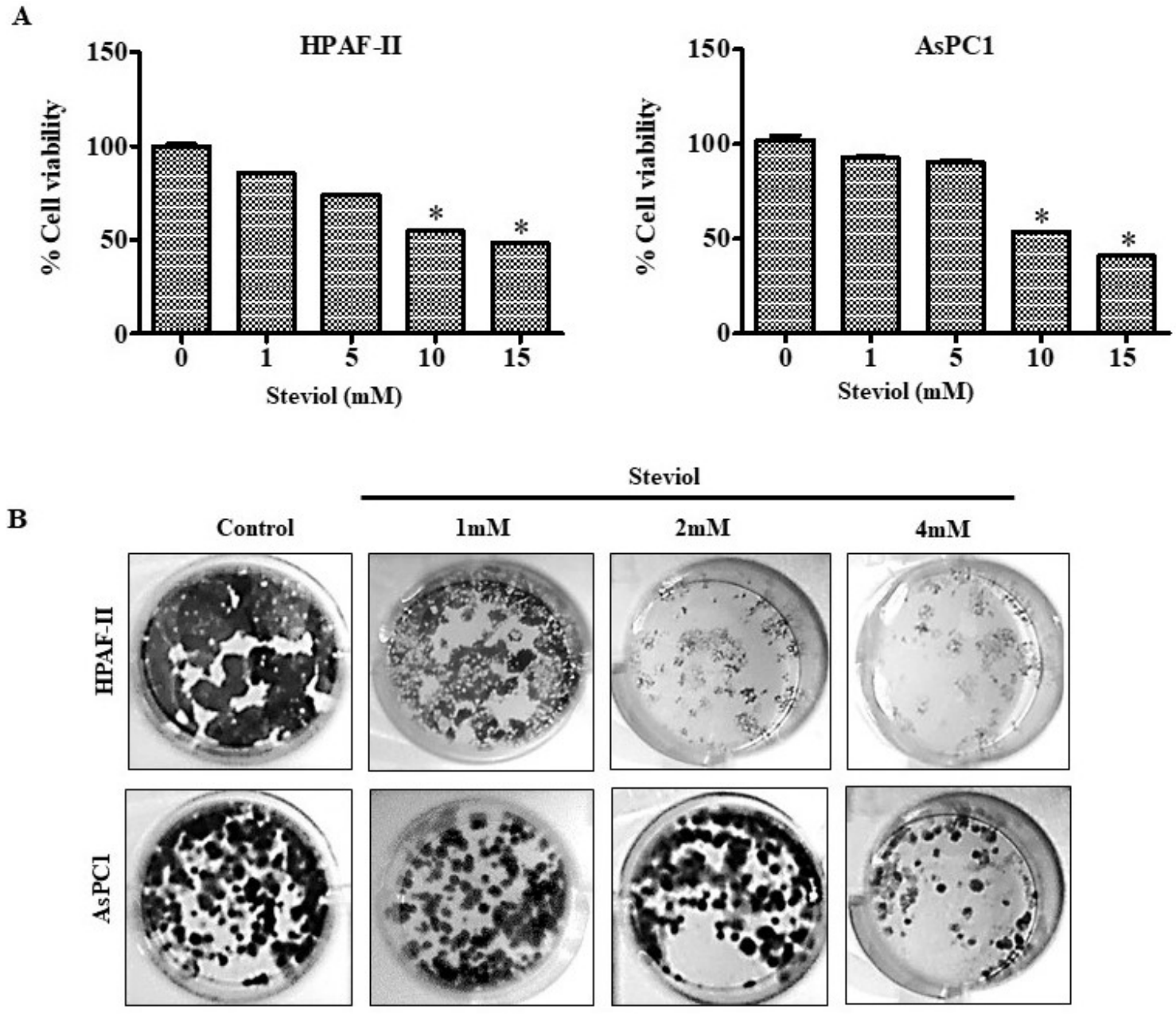

Figure 4-2. Steviol Inhibits Cell Proliferation and Clonogenic Potential of Pancreatic Cancer Cells

(A) Pancreatic cancer cells were treated with different concentrations of steviol $(0,1,5$, 10 and $15 \mathrm{mM}$ ), and incubated for 48 hours. Cell proliferation assay was terminated after addition of MTT reagent and recording the absorbance in HPAF-II and AsPC1 cells. (B). Colony formation assay was performed on pancreatic cancer cells. The cells were seeded and after 4 days, treatment with steviol was done at different concentrations $(0,1,2$ and $4 \mathrm{mM}$ ), and incubated for another 10 days. $n=3 ; * p<0.05$. 
A

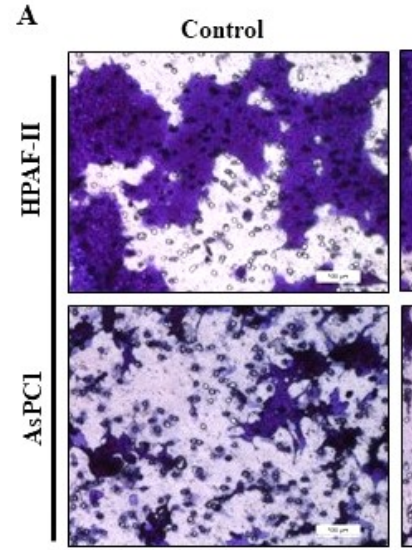

B

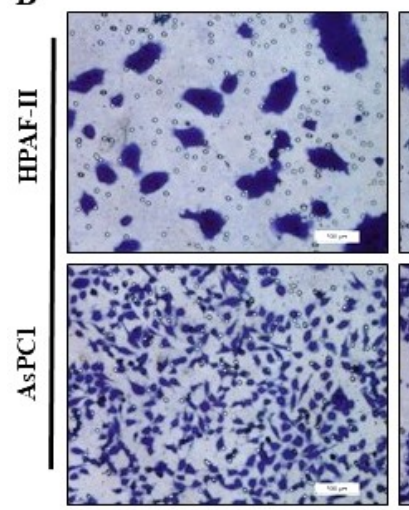

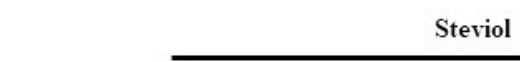

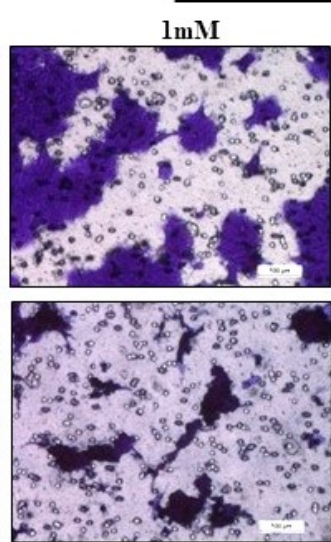

$\operatorname{lmM}$
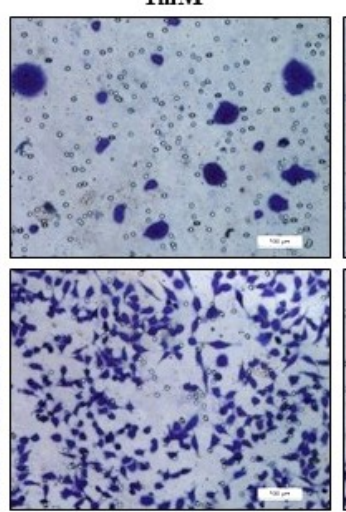

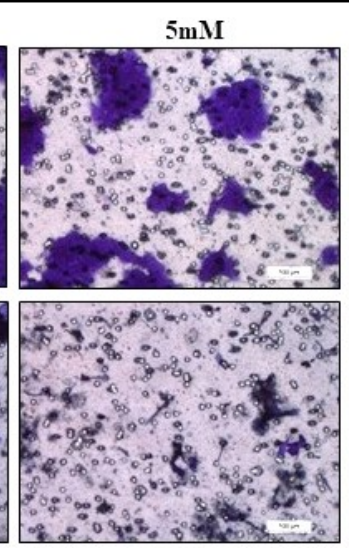

$5 \mathrm{mM}$

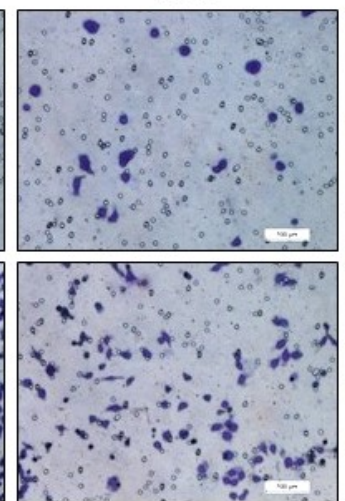

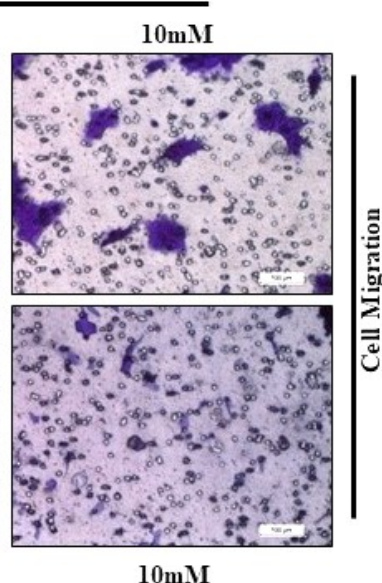

$10 \mathrm{mM}$

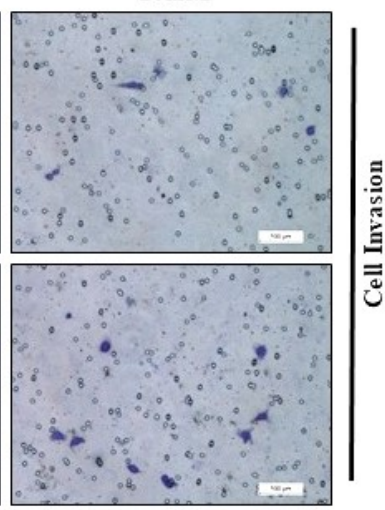

Figure 4-3. Steviol Inhibits Cell Migration and Cell Invasion in Pancreatic Cancer

(A) Boyden chamber migration assay was conducted on HPAF-II and AsPC1 cells. The cells were treated with $0,1,5$ and $10 \mathrm{mM}$ concentrations for 18 hours. Then the cells were fixed with $4 \%$ paraformaldehyde, stained with crystal violet and left for drying. Further, the images were captured (B) Matrigel invasion assay was performed on pancreatic cancer cells. The cells were treated with steviol at indicated concentrations for 24 hours. Thereafter, the cells were washed with PBS, fixed with methanol and stained using crystal violet. Upon drying, images were taken. 


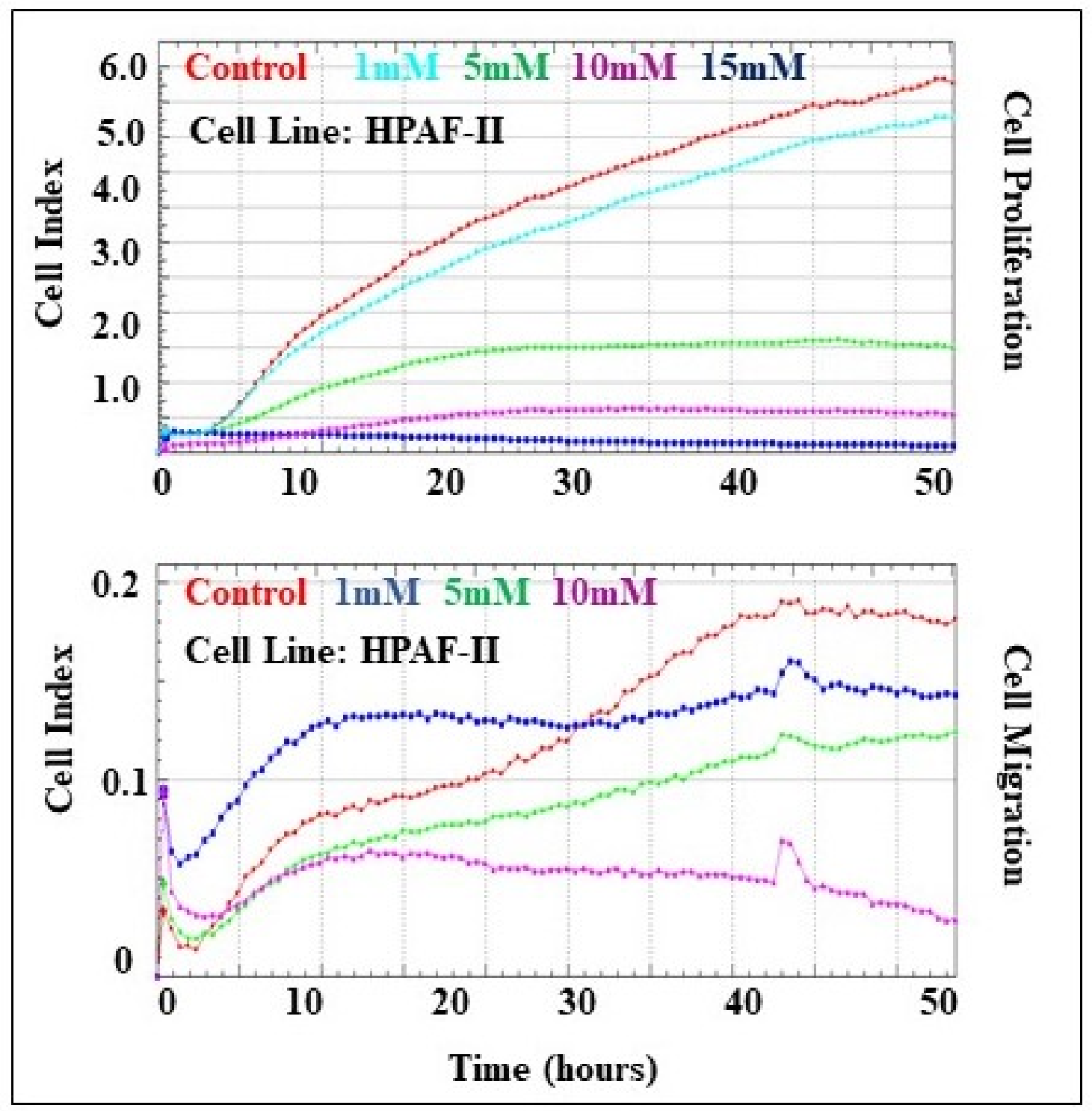

Figure 4-4. Steviol Causes Reduction in Cell Proliferation and Cell Migration in HPAF-II Cells

Pancreatic cancer cells (HPAF-II) were seeded and treated with different concentrations of steviol $(0,1,5,10$ and $15 \mathrm{mM})$. The cells were subjected to the xCELLigence instrument and cell proliferation and cell migration assay $(0,1,5,10 \mathrm{mM}$ of steviol) were conducted on the E-plate and CIM plates respectively. 
A

Time (hrs)

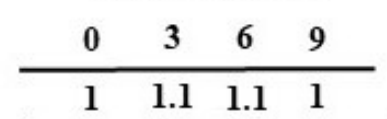

eIF4e

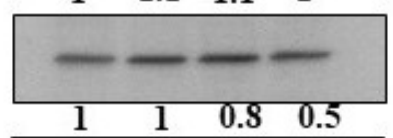

peIF4e

$4 \mathrm{eBPl}$

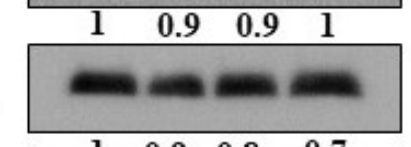

$\begin{array}{llll}1 & 0.9 & 0.8 & 0.7\end{array}$

p4eBP1

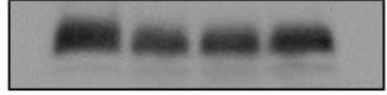

$\beta$-actin

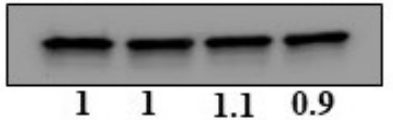

mTOR
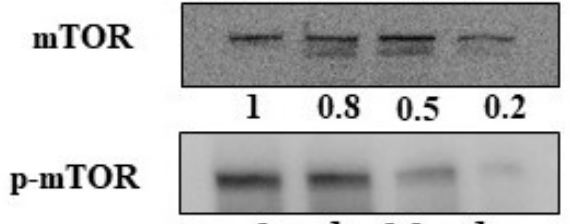

$\begin{array}{llll}1 & 1 & 1.1 & 1\end{array}$

eIF4B

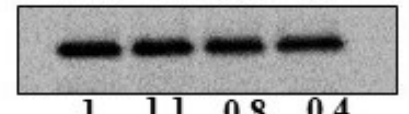

peIF4B

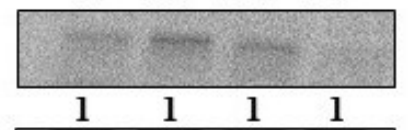

eIF4G

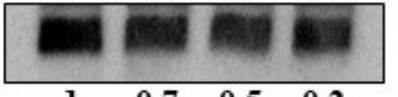

B

Time (hrs)

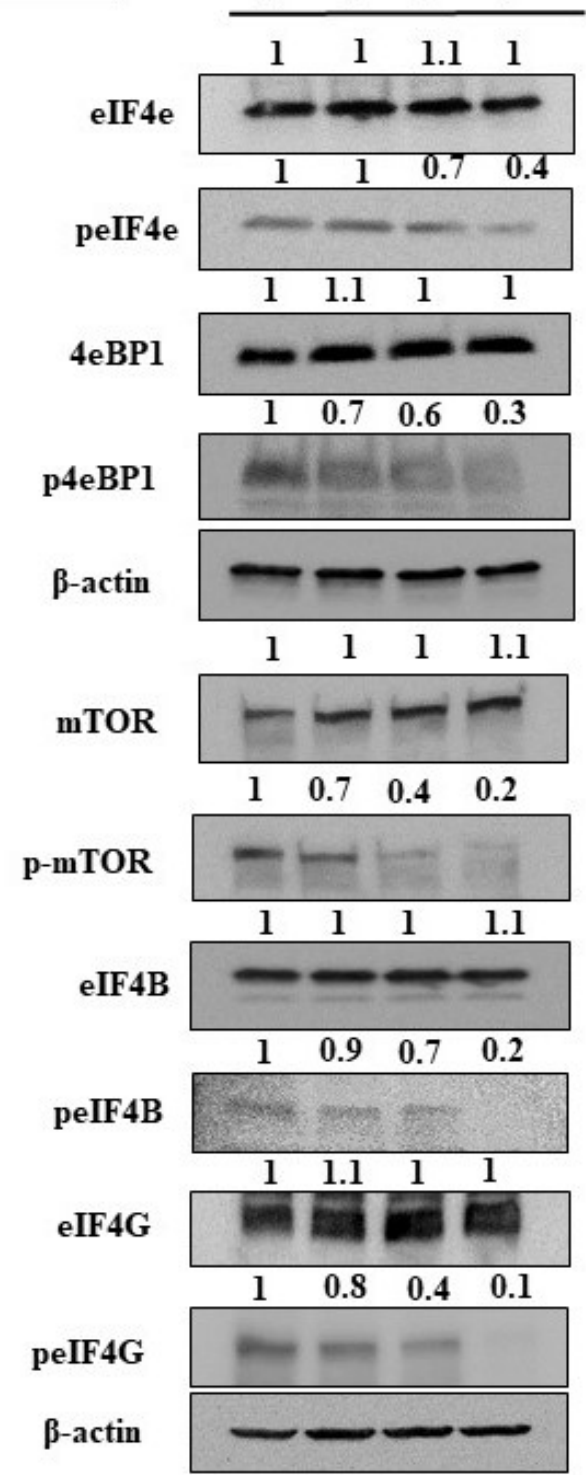

Figure 4-5. Steviol Impedes Translation Initiation

Cells were treated with steviol at different time points (0,3, 6 and 9 hours), and whole cell lysates were prepared. Western blotting was performed to analyze the expression level of proteins associated with translation initiation in (A) HPAF-II, and (B) AsPC1 cells. The numbers above the blots represent the relative band intensity after normalizing with $\beta$-actin (housekeeping gene). 
been documented that activation of eIF4E increases the key tumorigenic mRNAs translation in order to enhance oncogenic characteristics [189]. Steviol effectively decreased the phosphorylation status of this protein, in turn reducing the tumorigenic activity and delay in the translation process.

\section{Steviol Decreases Glucose Uptake and Lactate Secretion in PDAC Cells}

Cancer cells consume more glucose, which is metabolized by the glycolysis pathway, and lactate is the end product of glucose metabolism. Therefore, we investigated if steviol could lower the lactate production in PDAC cells. Our findings indicated decreased lactate secretion in both pancreatic cancer cells (Figure 4-6A).

The tumor cells undergo altered metabolism to support and sustain their upregulated growth and proliferative capabilities. Studies have reported about the potent anti-glycemic activity of Steviol precursor in rats and humans [131, 132]. However, its effect on the regulation of glucose metabolism in cancer cells has not been investigated. Therefore, we investigated its effect on glucose uptake/total glucose levels in pancreatic cancer cells Our results suggested that Steviol treatment reduced glucose consumption in pancreatic cancer cells (HPAF-II and AsPC1 cells) as compared to untreated cells (Figure 4-6B). These outcomes clearly suggest this compound having strong potential to suppress altered glucose metabolism in PDAC.

\section{Steviol Induces Apoptosis in Pancreatic Cancer Cells}

Apoptosis, commonly referred to as programmed cell death is a characteristic phenomenon of normal cells [190]. In abnormal conditions, the cells escape this process, which leads to tumorigenic condition. Several proteins are associated with the apoptotic pathway. Bax is one of the pro-apoptotic proteins crucially involved in this pathway. Also, other proteins such as Bcl-2, PARP and Bcl-xL are the significant anti-apoptotic proteins regulating these processes [191].

It was interesting to evaluate whether steviol has any role in apoptosis. Therefore, protein expression of apoptotic proteins was examined in pancreatic cancer cells after treatment with steviol in a time-dependent manner $(0,3,6$ and 9 hours) through immunoblotting. It was observed that the expression of Bax protein (pro-apoptotic) was upregulated in both HPAF-II and AsPC1 cell lines. Also, the expression level of important apoptosis-related proteins: Bcl-2 and Bcl-xL (anti-apoptotic proteins) decreased significantly in a dose-dependent manner. In addition, increased PARP cleavage (marker for early apoptosis) was observed after treatment with steviol. These results collectively indicated that steviol enhances apoptosis in pancreatic cancer as evident by the increased $\mathrm{Bax} / \mathrm{Bcl}-2$ ratio (Figure 4-7A, B). 
A

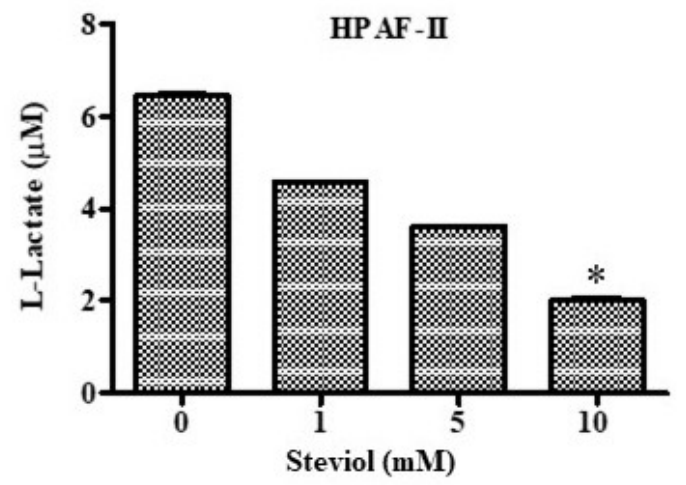

B

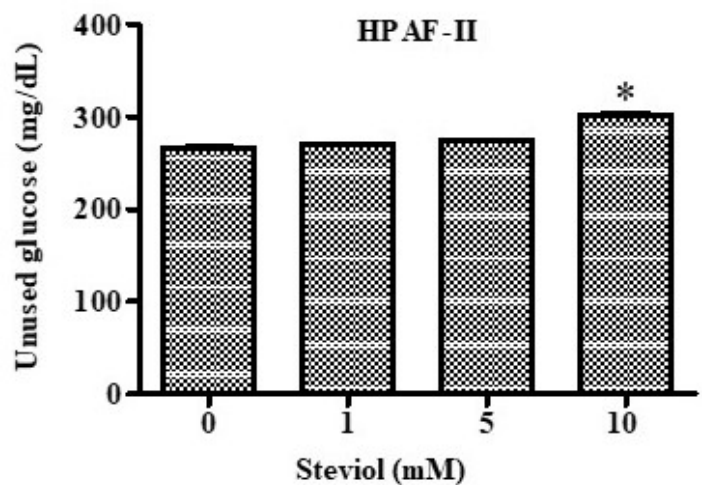

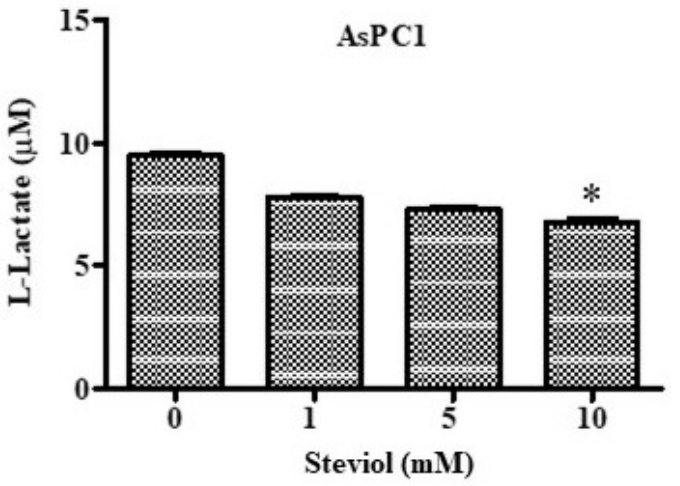

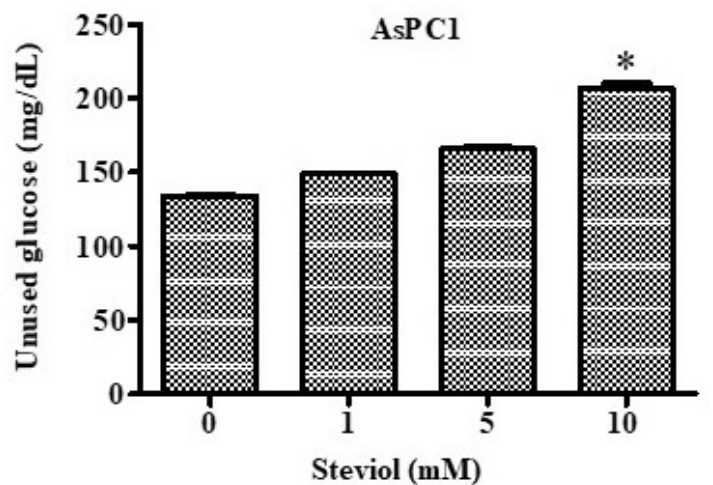

Figure 4-6. Steviol Inhibits Lactate Secretion and Glucose Uptake

Lactate and glucose assays were performed using commercially available kits in pancreatic cancer cells. Steviol treatment resulted in reduced lactate production and decreased glucose consumption in (A) HPAF-II, and (B) AsPC1 cells. $n=2 ;{ }^{*} p<0.05$. 
$\mathbf{A}$

Cells: HPAF-II

Time (hrs)

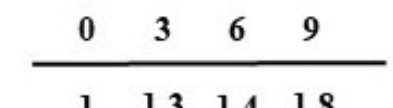

$\begin{array}{llll}1 & 1.3 & 1.4 & 1.8\end{array}$

Bax

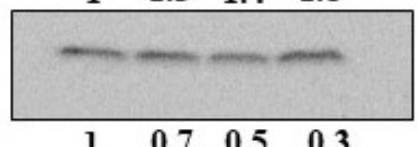

$\begin{array}{llll}1 & 0.7 & 0.5 & 0.3\end{array}$

Bcl-2

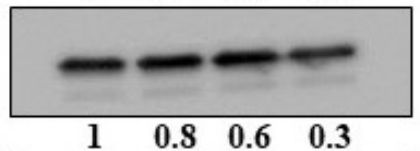

Bcl-xL
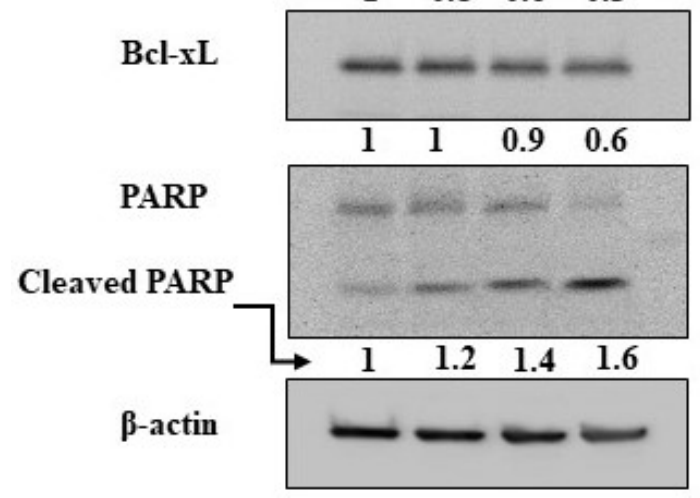

B

Cells: AsPC1
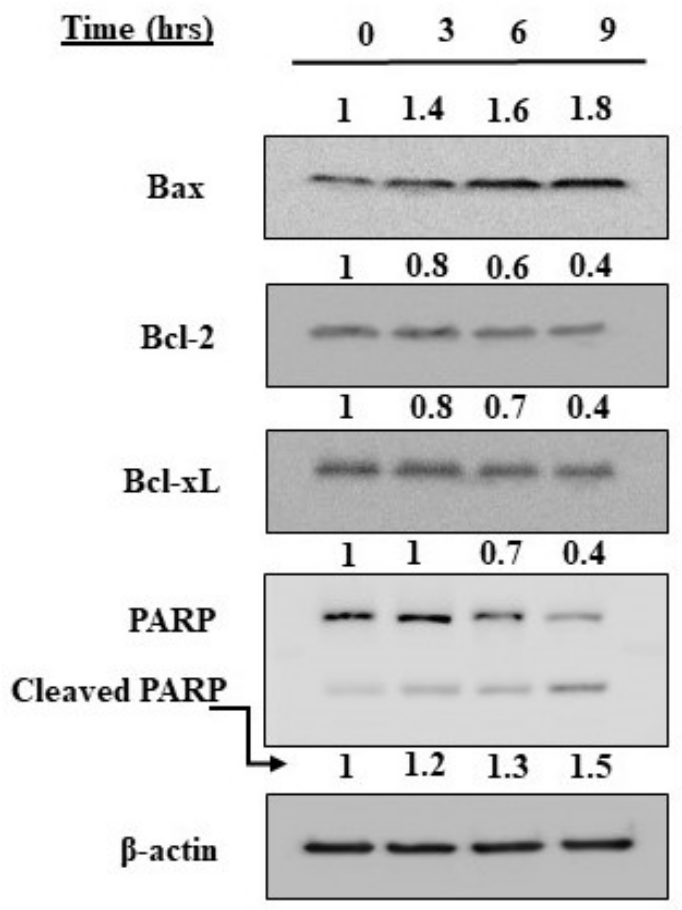

Figure 4-7. $\quad$ Steviol Inhibits Apoptosis in Pancreatic Cancer Cells

Pancreatic cancer cells were treated with steviol at different time points $(0,3,6$ and 9 hours), and western blotting was conducted after preparation of cell lysates. The expression level of important proteins associated with apoptotic pathway was evaluated in (A) HPAF-II, and (B) AsPC1 cells. Quantification of blots with respect to the loading control ( $\beta$-actin) was done as depicted by the numbers above the bands. 


\section{Steviol Treatment Results in G1 Cell Phase Arrest and Also Regulates the Proteins Involved}

In general, many drugs act by inducing cell cycle arrest of cancer cells. Therefore, to investigate the effect of steviol on G1 phase arrest on pancreatic cancer cells in a timedependent manner ( 0,12 and 24 hours), cell cycle experiment was performed. It was observed that steviol induced G1 arrest as depicted through the histogram graphs. The quantitative G1 population with respect to time is represented in HPAF-II and AsPC1 cells (Figure 4-8A, D).

In order to understand the underlying mechanism of the G1 phase arrest, the regulatory proteins which are associated with the G1 stage of cell cycle were evaluated to determine if steviol induced any inhibitory effects on them. Interestingly, the immunoblotting results demonstrated that the inhibitory proteins associated with cell cycle, mainly, p18 and p21 were significantly increased upon steviol treatment. On the other hand, Cyclin D1, which is critically involved in the progression of cell cycle [192], was remarkably downregulated in presence of steviol in comparison to the normal (untreated) cells. These observations suggest that steviol effectively modulates the important cell cycle associated proteins (Figure 4-9A, B).

\section{Discussion}

Pancreatic cancer is the most dreadful disease among cancers across the globe, with higher rates of cancer deaths in USA [1]. In this regard, there is an urgent need to reduce the death rates and develop fruitful therapeutic options to counteract this disease. A number of natural compounds with low toxicity profile have been investigated for cancer in recent years. Steviol is a natural sweetener which has a number of beneficial properties. As it is derived from a plant, it has low toxicity and thus is safe for use. Studies have suggested its role as an anti-diabetic and anti-inflammatory compound. Steviol has also been reported as an anti-cancer agent in different cancers [193, 194]. But in pancreatic cancer, its effect has not been well established yet. Therefore, this is the first study investigating the anti-tumorigenic potential of this plant product in pancreatic cancer.

Steviol inhibited the expression of oncogenic molecular determinants (MUC13 and PKD1), which also prompted us to investigate its role in deregulated glucose metabolism and also its anti-cancer effects in pancreatic cancer cells. In this research, it was elucidated that steviol suppresses pancreatic cancer proliferation and growth in two cell line model systems (HPAF-II and AsPC1). This compound also demonstrated its strong potential with respect to inhibition of the migration and invasion properties of the pancreatic cancer cells which was observed through the boyden chamber migration assay, and Matrigel invasion assay. The real-time xCELLigence assay also illustrated similar results and thus confirmed our findings. 


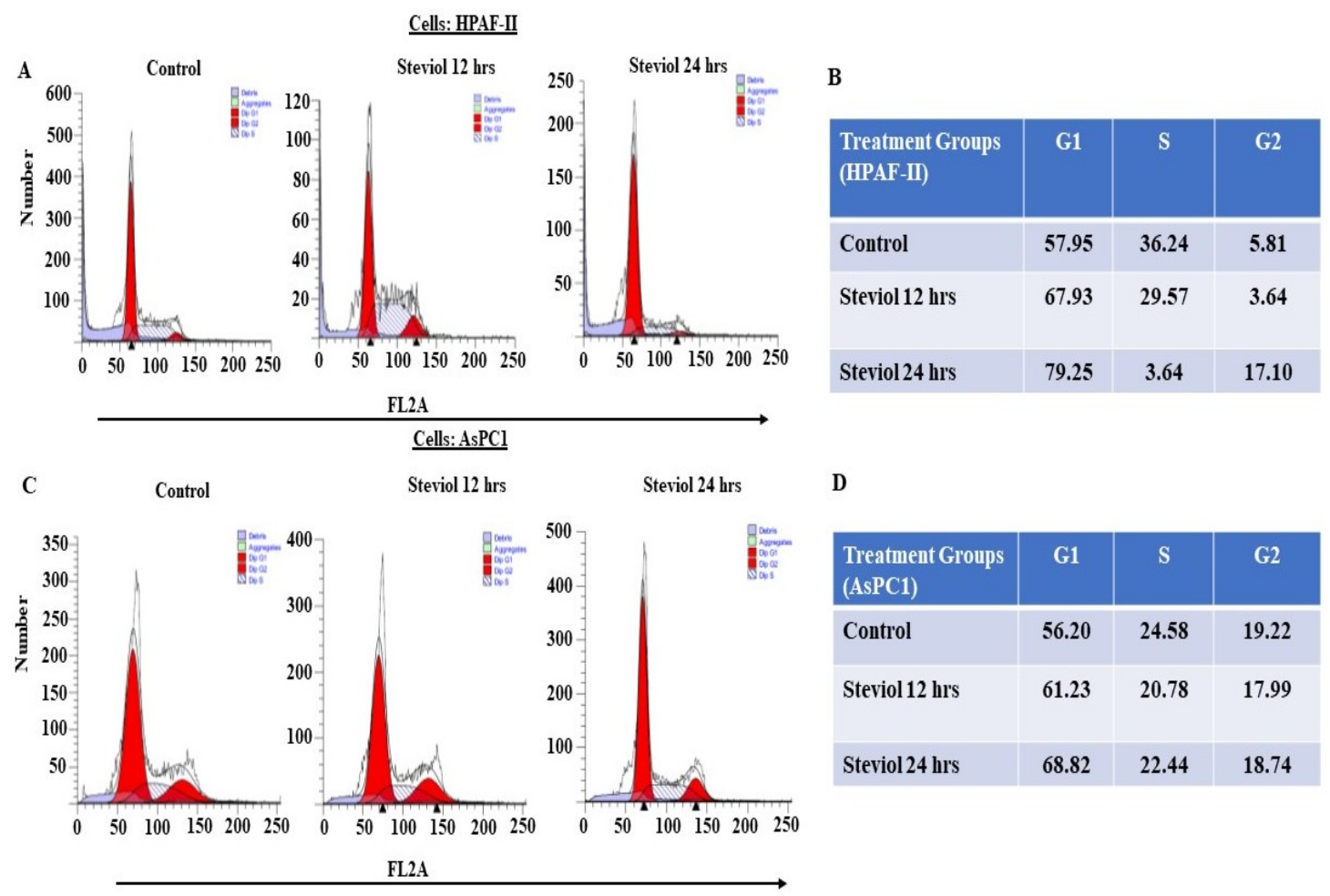

Figure 4-8. Steviol Treatment Leads to G1 Arrest

Pancreatic cancer cells were seeded and treated with steviol at different time points $(0,12$ and 24 hours). Cell cycle experiment was conducted to determine the effect of steviol on G1 cell cycle arrest in (A), (B) HPAF-II, and (C), (D) AsPC1 cells. 
$\mathbf{A}$

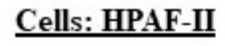

Time (hrs)

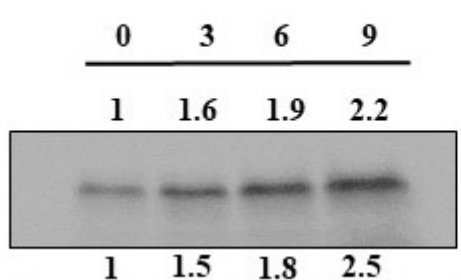

p21

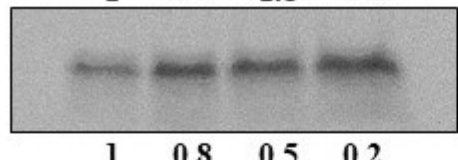

Cyclin D1

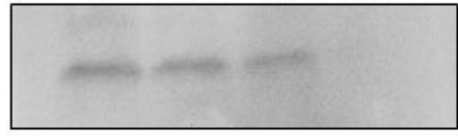

$\beta$-actin

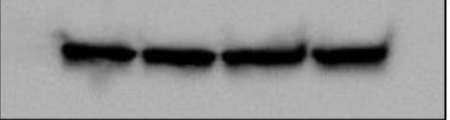

B

Cells: AsPC1

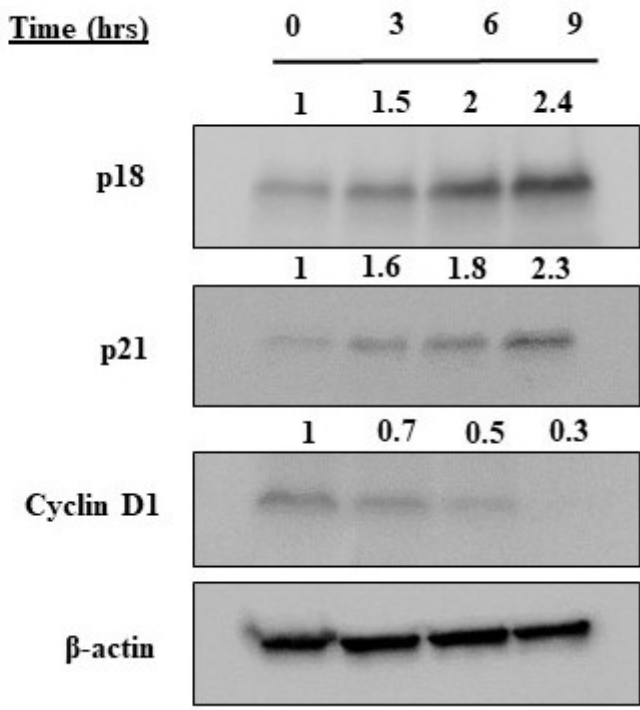

Figure 4-9. Steviol Modulates the Key Proteins Involved in G1 Cell Phase Arrest Pancreatic cancer cells were treated with steviol $(10 \mathrm{mM})$ at different time points $(0,3,6$ and 9 hours). Whole cell lysates were prepared and subjected to western blotting. The expression level of important cell cycle proteins (p18, p21 and Cyclin D1) were evaluated in (A) HPAF-II, and (B) AsPC1 cells. The numbers over the blots represent the relative quantification of band intensities with the internal control ( $\beta$-actin). 
As steviol is a plant product, it is expected to have multiple targets in oncogenic signaling pathways. As we observed increased expression of p4EBP1 protein in the cells treated with steviol, we wanted to investigate the changes in expression level of the proteins involved in the translation initiation apparatus. The key proteins associated with the translation machinery was observed to be downregulated in presence of steviol as indicated by our results. These proteins included: peIF4E, peIF4B, peIF4G, and p-mTOR, which are strongly associated with the translation-initiation process in eukaryotic cells. This in turn suggested that the activation of these key players in the process was disrupted by steviol treatment, further leading to inhibition of the translation mechanism itself. These findings are observed for the first time, which makes steviol a promising candidate for future studies.

In addition, our studies indicate that steviol inhibits apoptosis in pancreatic cancer cells, as suggested by the reduction in the expression level of apoptotic proteins such as Bcl-2 and PARP. Also, steviol leads to G1 cell cycle arrest, as observed through a rise in G1 phase population in a time-dependent manner. Our findings also indicate that steviol is very effective in regulating the prominent cell cycle associated proteins, namely p 18 , p21, as well as Cyclin D1 protein levels.

Altered glucose metabolism is an essential feature of pancreatic cancer. Therefore, it was imperative to investigate whether steviol can inhibit the aberrant metabolism. Our research demonstrated that steviol treatment with increasing concentrations, led to significant decrease in the glucose consumption in pancreatic cancer. Furthermore, the lactate secretion was also reduced when cells were treated with steviol in a concentration-dependent manner. These findings delineate the strong potential of steviol in overcoming the metabolic shift in pancreatic cancer.

Our results demonstrate that steviol has a significant role in modulating glucose metabolism, that might be involved in regulating the translation machinery through $\mathrm{mTOR} / \mathrm{AKT} / \mathrm{PI} 3 \mathrm{~K}$ altered mechanisms. We speculate that the alterations in glucose metabolism by steviol is the main factor which results in the suppression of translation machinery mechanism.

In conclusion, steviol, with regard to its beneficial effects of being minimally toxic and safe, can be considered as an effective anti-cancer agent, and be used in combination with other chemotherapeutic drugs to enhance the survival rate of pancreatic cancer patients. In the near future, it can be considered as an ideal candidate for more advanced research in this direction to develop promising therapeutic strategies to overcome this deadly disease. 


\section{CHAPTER 5. CONCLUSION AND FUTURE DIRECTIONS}

Pancreatic cancer cells demonstrate deregulated glucose metabolism and constitutive activation of several oncogenic signaling pathways leading to aggressive growth and metastatic phenotypes. These cells reshape their glucose metabolism in order to satiate the energy requirement in growing tumors and progression of metastasis. These metabolic adaptions promote the aerobic glycolytic events instead of oxidative phosphorylation resulting in the accumulation of lactate, subsequently altering the conditions in tissue microenvironment and driving it towards acidic milieu. Collectively, these developments trigger the excessive glucose metabolism in pancreatic tumors by facilitating cell proliferation in tumors. High glucose metabolism is a characteristic feature of pancreatic cancer and it fulfils the energy requirements of cancer cells. Due to the occurrence of these chronological events, the survival rate of the patients suffering from pancreatic cancer worsens. Studies have suggested that surgical removal of tumors from pancreatic cancer patients restored glucose metabolism [195]. Clinical data from another research signifies that diabetes mellitus in pancreatic cancer occurs as a result of the cancer itself [196].

However, there are only limited resources available about upstream key signaling molecules that regulate the downstream effector molecules which promote the activation of deregulated glucose metabolism. We believed that the identification of novel proteins in connection with aberrant glucose metabolism and therapeutic targeting would provide new dimensional approach for the treatment of pancreatic cancer patients with dysregulated glucose metabolism. MUC13 protein has been extensively studied in our lab, and we have identified its predominant role in pancreatic cancer development and progression. However, its role in altered metabolism had never been elucidated, which made it a probable candidate for our investigation. Also, Protein Kinase D molecules have been identified to be associated with glucose uptake/consumption in cardiac hypertrophy [137], but their role in cancer remains unidentified. Protein Kinase D1 (PKD1) is a kinase protein reported to be involved in hypoxic metabolism in other cancers [138], and has also been investigated extensively by our group. However, its role in pancreatic cancer and the associated mechanisms have not been very well investigated. Therefore, we were interested in investigating its association with elevated glucose metabolism which might further contribute to tumorigenesis and metabolic alterations.

With this note in chapter 2, we first evaluated the preeminent role of MUC13 as disruptor of glucose metabolism in pancreatic cancer cells. The results demonstrated that expression of MUC13 led to the activation/nuclear translocation of NFKB/p65 and phosphorylation of ІкВ. This resulted in the upregulation of downstream proteins (Glut$1, \mathrm{c}-\mathrm{Myc}$, and Bcl-2) those were involved in homeostasis of glucose metabolism. Our data demonstrate physical interaction of MUC13 with glucose receptor Glut-1. This interaction stimulated the downstream signaling leading to the promotion of enhanced intracellular glucose uptake. Our findings indicate that altered expressions of MUC13 governs the uptake of glucose, lactate accumulations and expression of metastatic genes. In addition, a NFאB inhibitor (sulfasalazine) interrupted the molecular interaction of 
Glut-1 and MUC13, that subsequently interfered with MUC13 induced altered glucose metabolism. Further, pancreatic ductal adenocarcinoma (PDAC) patient tissue samples displayed a decisive correlation and confirmed the association of MUC13 and Glut-1. These results deciphered mechanistic insight of MUC13 and its involvement in rewiring glucose metabolism that promoted the invasiveness of pancreatic cancer.

Concisely, we elucidated the functional role of MUC13 in rewiring of a discrete glucose metabolic network that initiates advantageous tumor microenvironment resulting in the activation of oncogenic signaling in pancreatic cancer cells that favored the metastasis. The inhibition of MUC13 by therapeutic agents specifically restrained the pancreatic tumor growth and subsequently interfered with the glycolytic influx. The dependence of pancreatic tumor growth and metastasis on MUC13-Glut-1 mediated glucose metabolism can be harnessed as molecular target for the therapeutic developments. Altogether, our study offered a propitious target for therapeutic interventions by reinstating the glucose metabolism in pancreatic cancer patients, thus being a promising strategy for extending the survival span in patients. Altogether, we conclusively demonstrated the role of MUC13 as a pro-regulator of dysregulated glucose metabolism and identified it as a clinical key molecule in pancreatic cancer patients with aberrant glucose metabolism. The discovery of this novel insight will encourage the development of newfangled therapeutic strategies for the pancreatic cancer patients suffering from dysregulated glucose metabolism.

Further, in search of associated proteins which are involved in dysregulated glucose metabolism in pancreatic cancer, we explored other possible candidates. In this view from chapter 3, we investigated the probable protein, Protein Kinase D1 (PKD1), with key functional role in rewiring of the glucose metabolism. Previously, few studies on PKD1 have inferred its role as an oncogenic protein in pancreatic cancer, but it's role in regulating glucose metabolism has not been completely elucidated. Therefore, in Chapter 3, we have shown expression profile of PKD1 in PDAC tissues and cells. The profiling results clearly indicates that PKD1 is overexpressed in pancreatic cancer tissues and cells compared to normal human tissues and cells. Our results also demonstrated that overexpression of PKD1 led to increased proliferation, invasion and migration of cells. In addition, the glucose uptake and lactate accumulations were escalated in PKD1 expressing cells. These results evidently affirmed the role of PKD1 in altering glucose metabolism. Additionally, in vitro results demonstrated that the invasive and migratory properties of PDAC cells were distinctly augmented in presence of induced PKD1 levels. These tumorigenic abilities of PKD1+/+ or PKD1+/- cells were amplified after the exposure with L-Lactate, a growth inducer while we observed converse effects during 2DG (negative deregulator) treatments.

Next, to comprehend the precise mechanism of PKD1 in dysregulating the glucose metabolism, we determined the several downstream key players. Among the all, proteins related to mTOR signaling were distinctly altered during the overexpression of PKD1. From protein profiling studies, we observed induced expression of $\mathrm{p}$-mTOR (ser2448), s6kinase, 4EBP1, and AKT in PKD1 overexpressing PDAC cells. Contrastingly, the phosphorylated levels of mTOR and ps6kinase expressions were declined during 
silencing of PKD1 and mTOR inhibition. These results deduced the physiological role of PKD1 in functioning of mTOR complex. In addition, lactate and glucose assays reconfirmed these results as glucose consumption and lactate secretion was reduced when raptor was silenced, but no difference was observed on silencing rictor in PKD1 expressing pancreatic cancer cells, annotating the functional relevance of mTORC1 in mediating aberrant glucose metabolism. Also, we observed the silencing of PKD1 chemo-sensitizes PDAC cells towards gemcitabine treatments. The overall outcomes of this study distinctly evidenced the role of PKD1 by causing the alterations in glucose metabolic network through mTORC1 complex, that apparently led to worsening of the disease. From these results we enacted PKD1 as prominent key player in causing aberrant glucose metabolism in pancreatic cancer.

Previously, in chapter 2 and 3, we elucidated the oncogenic roles of MUC13 and PKD1 respectively and their roles in dysregulated glucose metabolism in pancreatic cancer. However, in chapter 4 we propose the therapeutic perspective which specifically antagonize the functions of MUC13 and PKD1 proteins. The reason behind the selection of phytochemicals along the wide array of chemical agents is since it can minimize the toxicity issues and have potential to inhibit more than one molecular target in pancreatic cancer cells. In Chapter 4, we identified a drug- steviol, which is a plant product and thus has minimal to low toxicity. Steviol can be enacted as therapeutic agent in dual modes for reinstating glucose metabolism. Firstly, it can play as a food supplement that mimics glucose, that facilitates enhanced glucose uptake, and subsequently improves impaired glucose metabolism. Secondly, due to its therapeutic roles with accumulated intracellular levels exhibit potent anti-cancer effects. The anti-tumorigenic activity of steviol was evaluated through proliferation and other functional studies. Steviol exhibited dose dependent growth inhibitory effects on pancreatic cancer cells by restoring the dysregulated glucose metabolism and it also inhibited both MUC13 and PKD1 proteins. The molecular effects of steviol include the inhibition of translation-initiation complex that stalled the growth of cells and promoted the cell arrest at G1 phase in pancreatic cancer. From these events subsequently, cells were compelled to activate the apoptotic cascade resulting in cell death. Altogether, we proposed an efficient method therapy that effectively reconstructs the aberrant glucose metabolism and also targets MUC13 and PKD1 proteins in pancreatic cancer cells (Figure 5-1). As steviol is a non-toxic natural product, we believe that it can be used as an adjuvant in combination with known therapeutic agents of pancreatic cancer. Altogether, we can hope to decrease the lethality rates of pancreatic cancer patients by utilizing these effective strategies.

In the future, we can elucidate the role of these molecular determinants by using the MUC13/PKD1 overexpressing or knockdown pancreatic cancer cells. Moreover, their role in pancreatic carcinogenesis can be defined by genetic approach using Cre-Lox mediated technology. Also, as we expect that steviol can be used as a preventive agent, therefore, we can further investigate the chemo-preventive effects of Steviol against pancreatic cancer in an appropriate spontaneous mouse model (p48 Cre//KRas), which very closely represents pancreatic cancer progression in humans. This will enable us to delineate the molecular mechanisms and the in vivo effects of steviol. Also, outcome of 


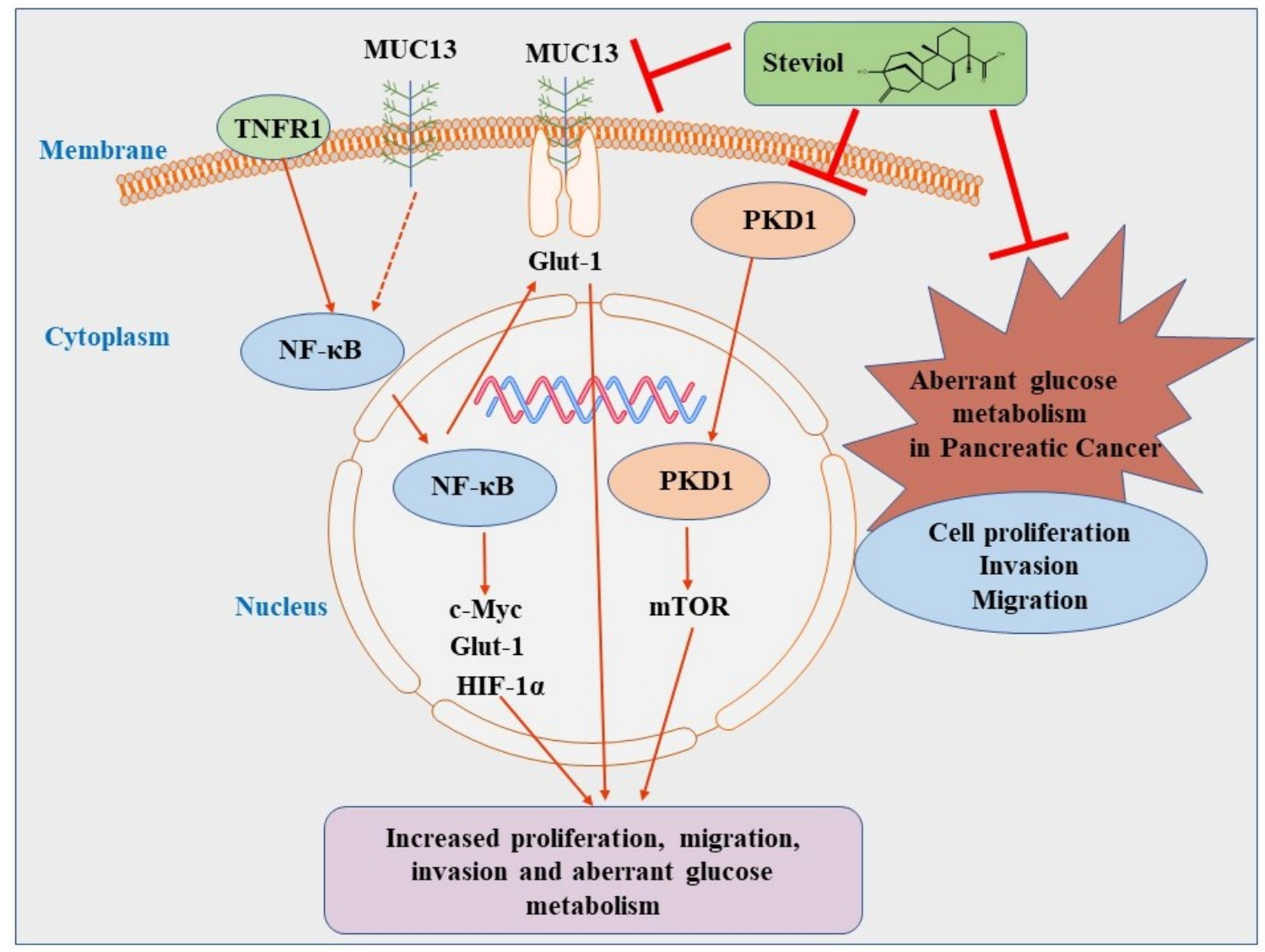

Figure 5-1. Schematic Diagram Representing the Overall Conclusion of the Study MUC13 and PKD1 were identified as essential contributing molecules for promoting dysregulated glucose metabolism in pancreatic cancer. Steviol, a natural plant product, demonstrated anti-cancer effects for the first time in pancreatic cancer. It also suppressed aberrant glucose metabolism and inhibited the expression of both MUC13 and PKD1 proteins, suggesting its strong therapeutic potential. 
this chemo-preventive study will set the stage for future phase I clinical trial against pancreatic cancer in humans. In this proposed study, Steviol (about $1000 \mathrm{mg}$, orally) can be given either to those patients who have high risk for developing pancreatic cancer or early stage cancer patients (only having PanIN lesions). These studies will provide better insights with respect to effectiveness of steviol in humans. 


\section{LIST OF REFERENCES}

1. Siegel, R.L., K.D. Miller, and A. Jemal, Cancer statistics, 2018. 2018. 68(1): p. 730 .

2. Del Chiaro, M., et al., Early detection and prevention of pancreatic cancer: is it really possible today? World J Gastroenterol, 2014. 20(34): p. 12118-31.

3. Kawada, K., K. Toda, and Y. Sakai, Targeting metabolic reprogramming in KRAS-driven cancers. Int J Clin Oncol, 2017. 22(4): p. 651-659.

4. Ferlay, J., et al., Estimating the global cancer incidence and mortality in 2018: GLOBOCAN sources and methods. 2018.

5. Hezel, A.F., et al., Genetics and biology of pancreatic ductal adenocarcinoma. Genes Dev, 2006. 20(10): p. 1218-49.

6. Lowenfels, A.B. and P. Maisonneuve, Epidemiology and risk factors for pancreatic cancer. Best Pract Res Clin Gastroenterol, 2006. 20(2): p. 197-209.

7. Tingstedt, B., C. Weitkamper, and R. Andersson, Early onset pancreatic cancer: a controlled trial. Ann Gastroenterol, 2011. 24(3): p. 206-212.

8. McGuigan, A., et al., Pancreatic cancer: A review of clinical diagnosis, epidemiology, treatment and outcomes. World J Gastroenterol, 2018. 24(43): p. 4846-4861.

9. Dasgupta, A., et al., Evaluating the Metabolic Alterations in Pancreatic Cancer. Methods Mol Biol, 2019. 1882: p. 221-228.

10. Bohl, C.E., et al., FDG-PET CT in the evaluation of primary and secondary pancreatic malignancies. 2018. 65(10): p. e27115.

11. Kumari, S., et al., MUC13 contributes to rewiring of glucose metabolism in pancreatic cancer. Oncogenesis, 2018. 7(2): p. 19.

12. Marie, S.K. and S.M. Shinjo, Metabolism and brain cancer. Clinics (Sao Paulo), 2011. 66 Suppl 1: p. 33-43.

13. Liberti, M.V. and J.W. Locasale, The Warburg Effect: How Does it Benefit Cancer Cells? Trends Biochem Sci, 2016. 41(3): p. 211-218.

14. Cohen, R., et al., Targeting cancer cell metabolism in pancreatic adenocarcinoma. Oncotarget, 2015. 6(19): p. 16832-47.

15. Hay, N., Reprogramming glucose metabolism in cancer: can it be exploited for cancer therapy? Nat Rev Cancer, 2016. 16(10): p. 635-49.

16. Valvona, C.J., et al., The Regulation and Function of Lactate Dehydrogenase A: Therapeutic Potential in Brain Tumor. Brain Pathol, 2016. 26(1): p. 3-17.

17. Doherty, J.R. and J.L. Cleveland, Targeting lactate metabolism for cancer therapeutics. J Clin Invest, 2013. 123(9): p. 3685-92.

18. Rong, Y., et al., Lactate dehydrogenase $A$ is overexpressed in pancreatic cancer and promotes the growth of pancreatic cancer cells. Tumour Biol, 2013. 34(3): p. 1523-30.

19. Wike-Hooley, J.L., J. Haveman, and H.S. Reinhold, The relevance of tumour $p H$ to the treatment of malignant disease. Radiother Oncol, 1984. 2(4): p. 343-66.

20. Halestrap, A.P. and M.C. Wilson, The monocarboxylate transporter family--role and regulation. IUBMB Life, 2012. 64(2): p. 109-19. 
21. Miranda-Goncalves, V., et al., Monocarboxylate transporters (MCTs) in gliomas: expression and exploitation as therapeutic targets. Neuro Oncol, 2013. 15(2): p. 172-88.

22. Fang, J., et al., The H+-linked monocarboxylate transporter (MCT1/SLC16A1): a potential therapeutic target for high-risk neuroblastoma. Mol Pharmacol, 2006. 70(6): p. 2108-15.

23. Pinheiro, C., et al., Increasing expression of monocarboxylate transporters 1 and 4 along progression to invasive cervical carcinoma. Int J Gynecol Pathol, 2008. 27(4): p. 568-74.

24. de Oliveira, A.T., et al., Co-expression of monocarboxylate transporter 1 (MCT1) and its chaperone (CD147) is associated with low survival in patients with gastrointestinal stromal tumors (GISTS). J Bioenerg Biomembr, 2012. 44(1): p. 171-8.

25. Pinheiro, C., et al., Increased expression of monocarboxylate transporters 1, 2 , and 4 in colorectal carcinomas. Virchows Arch, 2008. 452(2): p. 139-46.

26. Pinheiro, C., et al., Monocarboxylate transporter 1 is up-regulated in basal-like breast carcinoma. Histopathology, 2010. 56(7): p. 860-7.

27. Cui, J., et al., FOXM1 Promotes the Warburg Effect and Pancreatic Cancer Progression via Transactivation of LDHA Expression. Clinical cancer research : an official journal of the American Association for Cancer Research, 2014. 20(10): p. 2595-2606.

28. Timm, K.N., et al., Hyperpolarized [U-(2) H, U-(13) C]Glucose reports on glycolytic and pentose phosphate pathway activity in EL4 tumors and glycolytic activity in yeast cells. Magn Reson Med, 2015. 74(6): p. 1543-7.

29. Patra, K.C. and N. Hay, The pentose phosphate pathway and cancer. Trends Biochem Sci, 2014. 39(8): p. 347-54.

30. Boros, L.G., et al., Oxythiamine and dehydroepiandrosterone inhibit the nonoxidative synthesis of ribose and tumor cell proliferation. Cancer Res, 1997. 57(19): p. 4242-8.

31. Przybytkowski, E. and D.A. Averill-Bates, Correlation between glutathione and stimulation of the pentose phosphate cycle in situ in Chinese hamster ovary cells exposed to hydrogen peroxide. Arch Biochem Biophys, 1996. 325(1): p. 91-8.

32. Tuttle, S., et al., Glucose-6-phosphate dehydrogenase and the oxidative pentose phosphate cycle protect cells against apoptosis induced by low doses of ionizing radiation. Radiat Res, 2000. 153(6): p. 781-7.

33. Yeh, G.C., et al., Adriamycin resistance in human tumor cells associated with marked alteration in the regulation of the hexose monophosphate shunt and its response to oxidant stress. Cancer Res, 1987. 47(22): p. 5994-9.

34. Lai, G.M., et al., Contribution of glutathione and glutathione-dependent enzymes in the reversal of adriamycin resistance in colon carcinoma cell lines. Int $\mathbf{J}$ Cancer, 1991. 49(5): p. 688-95.

35. Pham, L.V., et al., Targeting the hexosamine biosynthetic pathway and O-linked $N$-acetylglucosamine cycling for therapeutic and imaging capabilities in diffuse large B-cell lymphoma. Oncotarget, 2016. 7(49): p. 80599-80611. 
36. Teo, C.F., E.E. Wollaston-Hayden, and L. Wells, Hexosamine flux, the O-GlcNAc modification, and the development of insulin resistance in adipocytes. Mol Cell Endocrinol, 2010. 318(1-2): p. 44-53.

37. Yang, C., et al., High expression of GFAT1 predicts poor prognosis in patients with pancreatic cancer. Sci Rep, 2016. 6: p. 39044.

38. Dong, X., et al., Glucose metabolism gene variants modulate the risk of pancreatic cancer. Cancer Prev Res (Phila), 2011. 4(5): p. 758-66.

39. Ma, Z., D.J. Vocadlo, and K. Vosseller, Hyper-O-GlcNAcylation is anti-apoptotic and maintains constitutive NF-kappaB activity in pancreatic cancer cells. J Biol Chem, 2013. 288(21): p. 15121-30.

40. Banerjee, S., et al., Triptolide-induced cell death in pancreatic cancer is mediated by O-GlcNAc modification of transcription factor Sp1. J Biol Chem, 2013. 288(47): p. 33927-38.

41. Zois, C.E., E. Favaro, and A.L. Harris, Glycogen metabolism in cancer. Biochem Pharmacol, 2014. 92(1): p. 3-11.

42. Amelio, I., et al., Serine and glycine metabolism in cancer. Trends in biochemical sciences, 2014. 39(4): p. 191-198.

43. Kottakis, F., et al., LKB1 loss links serine metabolism to DNA methylation and tumorigenesis. Nature, 2016. 539(7629): p. 390-395.

44. Kaida, H., et al., The correlation between FDG uptake and biological molecular markers in pancreatic cancer patients. Eur J Radiol, 2016. 85(10): p. 1804-10.

45. Swierczynski, J., A. Hebanowska, and T. Sledzinski, Role of abnormal lipid metabolism in development, progression, diagnosis and therapy of pancreatic cancer. World J Gastroenterol, 2014. 20(9): p. 2279-303.

46. Nishi, K., et al., Inhibition of Fatty Acid Synthesis Induces Apoptosis of Human Pancreatic Cancer Cells. Anticancer Res, 2016. 36(9): p. 4655-60.

47. Brandi, J., et al., Proteomic analysis of pancreatic cancer stem cells: Functional role of fatty acid synthesis and mevalonate pathways. J Proteomics, 2017. 150: p. 310-322.

48. Son, J., et al., Glutamine supports pancreatic cancer growth through a KRASregulated metabolic pathway. Nature, 2013. 496(7443): p. 101-5.

49. Meyer, K.A., et al., Adipocytes promote pancreatic cancer cell proliferation via glutamine transfer. Biochem Biophys Rep, 2016. 7: p. 144-149.

50. Jeong, S.M., et al., Enhanced mitochondrial glutamine anaplerosis suppresses pancreatic cancer growth through autophagy inhibition. Sci Rep, 2016. 6: p. 30767.

51. Locasale, J.W., Serine, glycine and one-carbon units: cancer metabolism in full circle. Nat Rev Cancer, 2013. 13(8): p. 572-83.

52. Almoguera, C., et al., Most human carcinomas of the exocrine pancreas contain mutant c-K-ras genes. Cell, 1988. 53(4): p. 549-54.

53. Ying, H., et al., Oncogenic Kras maintains pancreatic tumors through regulation of anabolic glucose metabolism. Cell, 2012. 149(3): p. 656-670.

54. Pramanik, K.C. and M.R. Makena, Advancement of NF-kappaB Signaling Pathway: A Novel Target in Pancreatic Cancer. 2018. 19(12).

55. Mann, K.M., et al., KRAS-related proteins in pancreatic cancer. Pharmacol Ther, 2016. 168: p. 29-42. 
56. Miller, D.M., et al., c-Myc and cancer metabolism. Clin Cancer Res, 2012. 18(20): p. 5546-53.

57. Miller, D.M., et al., c-Myc and cancer metabolism. Clinical cancer research : an official journal of the American Association for Cancer Research, 2012. 18(20): p. 5546-5553.

58. Shim, H., et al., c-Myc transactivation of $L D H$-A: implications for tumor metabolism and growth. Proc Natl Acad Sci U S A, 1997. 94(13): p. 6658-63.

59. Subramanian, A. and D.M. Miller, Structural analysis of alpha-enolase. Mapping the functional domains involved in down-regulation of the c-myc protooncogene. J Biol Chem, 2000. 275(8): p. 5958-65.

60. Hessmann, E., et al., MYC in pancreatic cancer: novel mechanistic insights and their translation into therapeutic strategies. Oncogene, 2016. 35(13): p. 1609-18.

61. Szablewski, L., Expression of glucose transporters in cancers. Biochim Biophys Acta, 2013. 1835(2): p. 164-9.

62. Krzeslak, A., et al., Expression of GLUT1 and GLUT3 glucose transporters in endometrial and breast cancers. Pathol Oncol Res, 2012. 18(3): p. 721-8.

63. Lu, K., et al., Expression and clinical significance of glucose transporter-1 in pancreatic cancer. Oncol Lett, 2016. 12(1): p. 243-249.

64. Alakus, H., et al., Variable $18 F$-fluorodeoxyglucose uptake in gastric cancer is associated with different levels of GLUT-1 expression. Nucl Med Commun, 2010. 31(6): p. 532-8.

65. Sharen, G., et al., Prognostic value of GLUT-1 expression in pancreatic cancer: results from 538 patients. Oncotarget, 2017. 8(12): p. 19760-19767.

66. Basturk, O., et al., GLUT-1 expression in pancreatic neoplasia: implications in pathogenesis, diagnosis, and prognosis. Pancreas, 2011. 40(2): p. 187-192.

67. Semenza, G.L., Regulation of mammalian O2 homeostasis by hypoxia-inducible factor 1. Annu Rev Cell Dev Biol, 1999. 15: p. 551-78.

68. Wenger, R.H., D.P. Stiehl, and G. Camenisch, Integration of oxygen signaling at the consensus HRE. Sci STKE, 2005. 2005(306): p. re12.

69. Matsumoto, S., et al., Imaging cycling tumor hypoxia. Cancer Res, 2010. 70(24): p. 10019-23.

70. Demchenko, Y.N. and W.M. Kuehl, A critical role for the NFkB pathway in multiple myeloma. Oncotarget, 2010. 1(1): p. 59-68.

71. Ersahin, T., N. Tuncbag, and R. Cetin-Atalay, The PI3K/AKT/mTOR interactive pathway. Mol Biosyst, 2015. 11(7): p. 1946-54.

72. Copp, J., G. Manning, and T. Hunter, TORC-specific phosphorylation of mammalian target of rapamycin (mTOR): phospho-Ser2481 is a marker for intact mTOR signaling complex 2. Cancer Res, 2009. 69(5): p. 1821-7.

73. Hall, M.N., mTOR-what does it do? Transplant Proc, 2008. 40(10 Suppl): p. S5-8.

74. Ashkenazi, A. and V.M. Dixit, Death receptors: signaling and modulation. Science, 1998. 281(5381): p. 1305-8.

75. Ling, J., et al., KrasG12D-Induced IKK2/B/NF- $\kappa B$ Activation by IL-1 $\alpha$ and $p 62$ Feedforward Loops Is Required for Development of Pancreatic Ductal Adenocarcinoma. Cancer Cell, 2012. 21(1): p. 105-120.

76. Kelley, S.K. and A. Ashkenazi, Targeting death receptors in cancer with Apo2L/TRAIL. Curr Opin Pharmacol, 2004. 4(4): p. 333-9. 
77. Bertsch, U., et al., Compartmentalization of TNF-related apoptosis-inducing ligand (TRAIL) death receptor functions: emerging role of nuclear TRAIL-R2. Cell death \& disease, 2014. 5(8): p. e1390-e1390.

78. Wang, X., The expanding role of mitochondria in apoptosis. Genes Dev, 2001. 15(22): p. 2922-33.

79. Ali, M.U., et al., Eukaryotic translation initiation factors and cancer. Tumour Biol, 2017. 39(6): p. 1010428317709805.

80. Sonenberg, N. and A.G. Hinnebusch, Regulation of translation initiation in eukaryotes: mechanisms and biological targets. Cell, 2009. 136(4): p. 731-45.

81. Ma, X.M. and J. Blenis, Molecular mechanisms of mTOR-mediated translational control. Nat Rev Mol Cell Biol, 2009. 10(5): p. 307-18.

82. Chauhan, S.C., et al., MUC13 mucin augments pancreatic tumorigenesis. Mol Cancer Ther, 2012. 11(1): p. 24-33.

83. Khan, S., et al., MUC13 interaction with receptor tyrosine kinase HER2 drives pancreatic ductal adenocarcinoma progression. Oncogene, 2017. 36(4): p. 491500.

84. Khan, S., et al., Clinical significance of MUC13 in pancreatic ductal adenocarcinoma. HPB (Oxford), 2018. 20(6): p. 563-572.

85. Maher, D.M., et al., Mucin 13: structure, function, and potential roles in cancer pathogenesis. Mol Cancer Res, 2011. 9(5): p. 531-537.

86. Sundram, V., S.C. Chauhan, and M. Jaggi, Emerging roles of protein kinase D1 in cancer. Mol Cancer Res, 2011. 9(8): p. 985-96.

87. Liou, G.Y., P. Storz, and M. Leitges, A bright future for protein kinase D1 as a drug target to prevent or treat pancreatic cancer. Mol Cell Oncol, 2016. 3(1): p. e1035477.

88. Doppler, H. and P. Storz, Mitochondrial and Oxidative Stress-Mediated Activation of Protein Kinase D1 and Its Importance in Pancreatic Cancer. Front Oncol, 2017. 7: p. 41.

89. Ochi, N., et al., Protein kinase D1 promotes anchorage-independent growth, invasion, and angiogenesis by human pancreatic cancer cells. J Cell Physiol, 2011. 226(4): p. 1074-81.

90. Aggarwal, M. and A.K. Mondal, Role of N-terminal hydrophobic region in modulating the subcellular localization and enzyme activity of the bisphosphate nucleotidase from Debaryomyces hansenii. Eukaryot Cell, 2006. 5(2): p. 262-71.

91. Lei, M., et al., Sampling protein conformations and pathways. J Comput Chem, 2004. 25(9): p. 1133-48.

92. Rozengurt, E., Protein kinase D signaling: multiple biological functions in health and disease. Physiology (Bethesda), 2011. 26(1): p. 23-33.

93. Alistar, A., et al., Safety and tolerability of the first-in-class agent CPI-613 in combination with modified FOLFIRINOX in patients with metastatic pancreatic cancer: a single-centre, open-label, dose-escalation, phase 1 trial. Lancet Oncol, 2017. 18(6): p. 770-778.

94. Passadouro, M. and H. Faneca, Managing Pancreatic Adenocarcinoma: A Special Focus in MicroRNA Gene Therapy. Int J Mol Sci, 2016. 17(5). 
95. Oettle, H., et al., Adjuvant chemotherapy with gemcitabine vs observation in patients undergoing curative-intent resection of pancreatic cancer: a randomized controlled trial. Jama, 2007. 297(3): p. 267-77.

96. Moore, M.J., et al., Erlotinib plus gemcitabine compared with gemcitabine alone in patients with advanced pancreatic cancer: a phase III trial of the National Cancer Institute of Canada Clinical Trials Group. J Clin Oncol, 2007. 25(15): p. 1960-6.

97. Pelzer, U., et al., Best supportive care (BSC) versus oxaliplatin, folinic acid and 5-fluorouracil (OFF) plus BSC in patients for second-line advanced pancreatic cancer: a phase III-study from the German CONKO-study group. Eur J Cancer, 2011. 47(11): p. 1676-81.

98. Von Hoff, D.D., et al., Increased survival in pancreatic cancer with nabpaclitaxel plus gemcitabine. N Engl J Med, 2013. 369(18): p. 1691-703.

99. Coleman, M.C., et al., 2-deoxy-D-glucose causes cytotoxicity, oxidative stress, and radiosensitization in pancreatic cancer. Free Radic Biol Med, 2008. 44(3): p. 322-31.

100. Chapiro, J., et al., Systemic delivery of microencapsulated 3-bromopyruvate for the therapy of pancreatic cancer. Clinical cancer research : an official journal of the American Association for Cancer Research, 2014. 20(24): p. 6406-6417.

101. Chapiro, J., et al., Systemic delivery of microencapsulated 3-bromopyruvate for the therapy of pancreatic cancer. Clin Cancer Res, 2014. 20(24): p. 6406-17.

102. Yao, J.C., et al., Everolimus for advanced pancreatic neuroendocrine tumors. N Engl J Med, 2011. 364(6): p. 514-23.

103. Jeong, W., et al., Pilot trial of EZN-2968, an antisense oligonucleotide inhibitor of hypoxia-inducible factor-1 alpha (HIF-1alpha), in patients with refractory solid tumors. Cancer Chemother Pharmacol, 2014. 73(2): p. 343-8.

104. Liang, C., et al., Energy sources identify metabolic phenotypes in pancreatic cancer. Acta Biochim Biophys Sin (Shanghai), 2016. 48(11): p. 969-979.

105. Chen, H., et al., A perylene derivative regulates HIF-lalpha and Stat3 signaling pathways. Bioorg Med Chem, 2014. 22(4): p. 1496-505.

106. He, M., et al., Chidamide Inhibits Aerobic Metabolism to Induce Pancreatic Cancer Cell Growth Arrest by Promoting Mcl-1 Degradation. PLoS One, 2016. 11(11): p. e0166896.

107. Zhao, B. and T. He, Chidamide, a histone deacetylase inhibitor, functions as a tumor inhibitor by modulating the ratio of Bax/Bcl-2 and $\mathrm{P} 21$ in pancreatic cancer. Oncol Rep, 2015. 33(1): p. 304-10.

108. Dong, M., et al., Phase I study of chidamide (CS055/HBI-8000), a new histone deacetylase inhibitor, in patients with advanced solid tumors and lymphomas. Cancer Chemother Pharmacol, 2012. 69(6): p. 1413-22.

109. Fujiwara, Y., et al., Phase 1 study of galunisertib, a TGF-beta receptor I kinase inhibitor, in Japanese patients with advanced solid tumors. Cancer Chemother Pharmacol, 2015. 76(6): p. 1143-52.

110. Ganapathy-Kanniappan, S. and J.F. Geschwind, Tumor glycolysis as a target for cancer therapy: progress and prospects. Mol Cancer, 2013. 12: p. 152. 
111. Chakrabarti, G., et al., Targeting glutamine metabolism sensitizes pancreatic cancer to PARP-driven metabolic catastrophe induced by ss-lapachone. Cancer Metab, 2015. 3: p. 12.

112. Lebovitz, C.B., et al., Precision autophagy: Will the next wave of selective autophagy markers and specific autophagy inhibitors feed clinical pipelines? Autophagy, 2015. 11(10): p. 1949-52.

113. Pollak, M.N., Investigating Metformin for Cancer Prevention and Treatment: The End of the Beginning. Cancer Discovery, 2012. 2(9): p. 778-790.

114. Albini, A., et al., Cancer Prevention and Interception: A New Era for Chemopreventive Approaches. Clin Cancer Res, 2016. 22(17): p. 4322-7.

115. Elgogary, A., et al., Combination therapy with BPTES nanoparticles and metformin targets the metabolic heterogeneity of pancreatic cancer. Proc Natl Acad Sci U S A, 2016. 113(36): p. E5328-36.

116. Price, G.S., et al., Pharmacokinetics and toxicity of oral and intravenous lonidamine in dogs. Cancer Chemother Pharmacol, 1996. 38(2): p. 129-35.

117. Zhong, D., et al., The glycolytic inhibitor 2-deoxyglucose activates multiple prosurvival pathways through IGF1R. J Biol Chem, 2009. 284(35): p. 23225-33.

118. Maher, J.C., et al., Hypoxia-inducible factor-1 confers resistance to the glycolytic inhibitor 2-deoxy-D-glucose. Mol Cancer Ther, 2007. 6(2): p. 732-41.

119. Maschek, G., et al., 2-deoxy-D-glucose increases the efficacy of adriamycin and paclitaxel in human osteosarcoma and non-small cell lung cancers in vivo. Cancer Res, 2004. 64(1): p. 31-4.

120. Dwarakanath, B. and V. Jain, Targeting glucose metabolism with 2-deoxy-Dglucose for improving cancer therapy. Future Oncol, 2009. 5(5): p. 581-5.

121. Yue, W., et al., Repurposing of metformin and aspirin by targeting AMPK-mTOR and inflammation for pancreatic cancer prevention and treatment. Cancer Prev Res (Phila), 2014. 7(4): p. 388-97.

122. Yue, W., et al., Transcriptomic analysis of pancreatic cancer cells in response to metformin and aspirin: an implication of synergy. Sci Rep, 2015. 5: p. 13390.

123. Di Gangi, I.M., et al., Metabolomic profile in pancreatic cancer patients: a consensus-based approach to identify highly discriminating metabolites. Oncotarget, 2016. 7(5): p. 5815-29.

124. Le, A., et al., Inhibition of lactate dehydrogenase A induces oxidative stress and inhibits tumor progression. Proc Natl Acad Sci U S A, 2010. 107(5): p. 2037-42.

125. Cazzaniga, M. and B. Bonanni, Relationship Between Metabolic Reprogramming and Mitochondrial Activity in Cancer Cells. Understanding The Anticancer Effect of Metformin and Its Clinical Implications. Anticancer Res, 2015. 35(11): p. 5789-96.

126. Mohd-Radzman, N.H., et al., Stevioside from Stevia rebaudiana Bertoni Increases Insulin Sensitivity in 3T3-L1 Adipocytes. Evid Based Complement Alternat Med, 2013. 2013: p. 938081.

127. Boonkaewwan, C. and A. Burodom, Anti-inflammatory and immunomodulatory activities of stevioside and steviol on colonic epithelial cells. J Sci Food Agric, 2013.

128. Chatsudthipong, V. and C. Muanprasat, Stevioside and related compounds: therapeutic benefits beyond sweetness. Pharmacol Ther, 2009. 121(1): p. 41-54. 
129. Wu, Y., et al., Stevioside methanol tetra-solvate. Acta Crystallogr Sect E Struct Rep Online, 2013. 69(Pt 3): p. o410-1.

130. Geuns, J.M., et al., Metabolism of stevioside in pigs and intestinal absorption characteristics of stevioside, rebaudioside A and steviol. Food Chem Toxicol, 2003. 41(11): p. 1599-607.

131. Gregersen, S., et al., Antihyperglycemic effects of stevioside in type 2 diabetic subjects. Metabolism, 2004. 53(1): p. 73-6.

132. Jeppesen, P.B., et al., Antihyperglycemic and blood pressure-reducing effects of stevioside in the diabetic Goto-Kakizaki rat. Metabolism, 2003. 52(3): p. 372-8.

133. Sousa, C.M. and A.C. Kimmelman, The complex landscape of pancreatic cancer metabolism. Carcinogenesis, 2014. 35(7): p. 1441-1450.

134. Shukla, S.K., et al., MUC1 and HIF-1alpha Signaling Crosstalk Induces Anabolic Glucose Metabolism to Impart Gemcitabine Resistance to Pancreatic Cancer. Cancer Cell, 2017. 32(1): p. 71-87.e7.

135. Chaika, N.V., et al., MUC1 mucin stabilizes and activates hypoxia-inducible factor 1 alpha to regulate metabolism in pancreatic cancer. Proceedings of the National Academy of Sciences, 2012. 109(34): p. 13787-13792.

136. Shukla, S.K., et al., MUC16-mediated activation of mTOR and c-Myc reprograms pancreatic cancer metabolism. Oncotarget, 2015. 6(22): p. 19118-19131.

137. Simsek Papur, O., et al., Acute and Chronic Effects of Protein Kinase-D Signaling on Cardiac Energy Metabolism. Front Cardiovasc Med, 2018. 5: p. 65.

138. Chen, J., et al., Protein kinase D1 regulates hypoxic metabolism through HIFlalpha and glycolytic enzymes incancer cells. Oncol Rep, 2018. 40(2): p. 10731082.

139. Siegel, R.L., K.D. Miller, and A. Jemal, Cancer statistics, 2016. CA Cancer J Clin, 2016. 66(1): p. 7-30.

140. Cairns, R.A., I.S. Harris, and T.W. Mak, Regulation of cancer cell metabolism. Nat Rev Cancer, 2011. 11(2): p. 85-95.

141. Weinstein, I.B. and A. Joe, Oncogene addiction. Cancer Res, 2008. 68(9): p. 3077-80; discussion 3080.

142. Estrella, V., et al., Acidity generated by the tumor microenvironment drives local invasion. Cancer Res, 2013. 73(5): p. 1524-35.

143. Ojelabi, O., et al., Novel small molecule, WZB117, competitively inhibit GLUT1mediated glucose transport to halt cancer growth. The FASEB Journal, 2016. 30(1_supplement): p. 1099.1-1099.1.

144. Walenta, S., et al., High lactate levels predict likelihood of metastases, tumor recurrence, and restricted patient survival in human cervical cancers. Cancer Res, 2000. 60(4): p. 916-21.

145. Ying, H., et al., Oncogenic Kras maintains pancreatic tumors through regulation of anabolic glucose metabolism. Cell, 2012. 149(3): p. 656-70.

146. Chan, A.K., J.I. Bruce, and A.K. Siriwardena, Glucose metabolic phenotype of pancreatic cancer. World J Gastroenterol, 2016. 22(12): p. 3471-85.

147. Mauro, C., et al., NF-kappaB controls energy homeostasis and metabolic adaptation by upregulating mitochondrial respiration. Nat Cell Biol, 2011. 13(10): p. 1272-9. 
148. Kaur, S., et al., Mucins in pancreatic cancer and its microenvironment. Nat Rev Gastroenterol Hepatol, 2013. 10(10): p. 607-20.

149. Khan, S., et al., Ormeloxifene suppresses desmoplasia and enhances sensitivity of gemcitabine in pancreatic cancer. Cancer Res, 2015. 75(11): p. 2292-304.

150. Khan, S., et al., MicroRNA-145 targets MUC13 and suppresses growth and invasion of pancreatic cancer. Oncotarget, 2014. 5(17): p. 7599-609.

151. Jaggi, M., et al., E-cadherin phosphorylation by protein kinase D1/protein kinase $C\{m u\}$ is associated with altered cellular aggregation and motility in prostate cancer. Cancer Res, 2005. 65(2): p. 483-92.

152. Yallapu, M.M., et al., Novel curcumin-loaded magnetic nanoparticles for pancreatic cancer treatment. Mol Cancer Ther, 2013. 12(8): p. 1471-80.

153. DeBerardinis, R.J., Is cancer a disease of abnormal cellular metabolism? New angles on an old idea. Genet Med, 2008. 10(11): p. 767-77.

154. Khan, S., et al., A novel cyano derivative of 11-keto-beta-boswellic acid causes apoptotic death by disrupting PI3K/AKT/Hsp-90 cascade, mitochondrial integrity, and other cell survival signaling events in HL-60 cells. Mol Carcinog, 2012. 51(9): p. 679-95.

155. Sheng, Y.H., et al., MUC13 protects colorectal cancer cells from death by activating the NF-kappaB pathway and is a potential therapeutic target. Oncogene, 2017. 36(5): p. 700-713.

156. Wahl, C., et al., Sulfasalazine: a potent and specific inhibitor of nuclear factor kappa B. J Clin Invest, 1998. 101(5): p. 1163-74.

157. Levine, A.J. and A.M. Puzio-Kuter, The control of the metabolic switch in cancers by oncogenes and tumor suppressor genes. Science, 2010. 330(6009): p. 1340-4.

158. Yamamoto, T., et al., Over-expression of facilitative glucose transporter genes in human cancer. Biochem Biophys Res Commun, 1990. 170(1): p. 223-30.

159. Maher, J.C., et al., Differential sensitivity to 2-deoxy-D-glucose between two pancreatic cell lines correlates with GLUT-1 expression. Pancreas, 2005. 30(2): p. e34-9.

160. Panchamoorthy, G., et al., A monoclonal antibody against the oncogenic mucin 1 cytoplasmic domain. Hybridoma (Larchmt), 2011. 30(6): p. 531-5.

161. Yin, G., et al., mTOR complex 1 signalling regulates the balance between lipid synthesis and oxidation in hypoxia lymphocytes. Bioscience reports, 2017. 37(1): p. BSR20160479.

162. Shi, D., et al., Glycolysis inhibition via mTOR suppression is a key step in cardamonin-induced autophagy in SKOV3 cells. BMC complementary and alternative medicine, 2018. 18(1): p. 317-317.

163. Jaggi, M., et al., Protein kinase C mu is down-regulated in androgen-independent prostate cancer. Biochem Biophys Res Commun, 2003. 307(2): p. 254-60.

164. Doppler, H., et al., The PRKD1 promoter is a target of the KRas-NF-kappaB pathway in pancreatic cancer. Sci Rep, 2016. 6: p. 33758.

165. Liou, G.Y., et al., Mutant KRas-Induced Mitochondrial Oxidative Stress in Acinar Cells Upregulates EGFR Signaling to Drive Formation of Pancreatic Precancerous Lesions. Cell Rep, 2016. 14(10): p. 2325-36. 
166. Liou, G.Y., et al., Protein kinase D1 drives pancreatic acinar cell reprogramming and progression to intraepithelial neoplasia. Nat Commun, 2015. 6: p. 6200.

167. Mao, Z. and W. Zhang, Role of mTOR in Glucose and Lipid Metabolism. International journal of molecular sciences, 2018. 19(7): p. 2043.

168. Khan, S., et al., Ormeloxifene Suppresses Desmoplasia and Enhances Sensitivity of Gemcitabine in Pancreatic Cancer. Cancer Res, 2015.

169. Khan, S., et al., Ormeloxifene Suppresses Desmoplasia and Enhances Sensitivity of Gemcitabine in Pancreatic Cancer. Cancer Research, 2015. 75(11): p. 22922304.

170. Yallapu, M.M., et al., Novel Curcumin Loaded Magnetic Nanoparticles for Pancreatic Cancer Treatment. Molecular Cancer Therapeutics, 2013.

171. Guha, S., et al., Role of protein kinase D signaling in pancreatic cancer. Biochemical pharmacology, 2010. 80(12): p. 1946-1954.

172. Liou, G.-Y., et al., Protein kinase D1 drives pancreatic acinar cell reprogramming and progression to intraepithelial neoplasia. Nature communications, 2015. 6: p. 6200-6200.

173. Adekola, K., S.T. Rosen, and M. Shanmugam, Glucose transporters in cancer metabolism. Curr Opin Oncol, 2012. 24(6): p. 650-4.

174. Nishimoto, A., et al., HIF-1alpha activation under glucose deprivation plays a central role in the acquisition of anti-apoptosis in human colon cancer cells. Int $\mathbf{J}$ Oncol, 2014. 44(6): p. 2077-84.

175. Mao, L., et al., Berberine decelerates glucose metabolism via suppression of mTORdependent HIF lalpha protein synthesis in colon cancer cells. Oncol Rep, 2018. 39(5): p. 2436-2442.

176. Mao, Z. and W. Zhang, Role of mTOR in Glucose and Lipid Metabolism. 2018. 19(7).

177. Newsholme, E.A. and M. Board, Application of metabolic-control logic to fuel utilization and its significance in tumor cells. Adv Enzyme Regul, 1991. 31: p. 225-46.

178. Laplante, M. and D.M. Sabatini, mTOR signaling in growth control and disease. Cell, 2012. 149(2): p. 274-93.

179. Mi, C., et al., 4',6-dihydroxy-4-methoxyisoaurone inhibits the HIF-1alpha pathway through inhibition of Akt/mTOR/p70S6K/4E-BP1 phosphorylation. $\mathrm{J}$ Pharmacol Sci, 2014. 125(2): p. 193-201.

180. Duvel, K., et al., Activation of a metabolic gene regulatory network downstream of mTOR complex 1. Mol Cell, 2010. 39(2): p. 171-83.

181. Malm, S.W., et al., The anti-tumor efficacy of 2-deoxyglucose and D-allose are enhanced with $\mathrm{p} 38$ inhibition in pancreatic and ovarian cell lines. J Exp Clin Cancer Res, 2015. 34: p. 31.

182. Alayev, A. and M.K. Holz, mTOR signaling for biological control and cancer. J Cell Physiol, 2013. 228(8): p. 1658-64.

183. Spilka, R., et al., Eukaryotic translation initiation factors in cancer development and progression. Cancer Lett, 2013. 340(1): p. 9-21.

184. D'Abronzo, L.S. and P.M. Ghosh, eIF4E Phosphorylation in Prostate Cancer. Neoplasia, 2018. 20(6): p. 563-573. 
185. Mattison, J.A., et al., Impact of caloric restriction on health and survival in rhesus monkeys from the NIA study. Nature, 2012. 489(7415): p. 318-21.

186. Slattery, M.L., et al., Dietary sugar and colon cancer. Cancer Epidemiol Biomarkers Prev, 1997. 6(9): p. 677-85.

187. Fiechter, A. and F.K. Gmunder, Metabolic control of glucose degradation in yeast and tumor cells. Adv Biochem Eng Biotechnol, 1989. 39: p. 1-28.

188. Giuliani, C.M. and C.R. Dass, Metabolic stress and cancer: is autophagy the common denominator and a feasible target? J Pharm Pharmacol, 2013.

189. Gingras, A.C., B. Raught, and N. Sonenberg, eIF4 initiation factors: effectors of $m R N A$ recruitment to ribosomes and regulators of translation. Annu Rev Biochem, 1999. 68: p. 913-63.

190. Kaczanowski, S., Apoptosis: its origin, history, maintenance and the medical implications for cancer and aging. Phys Biol, 2016. 13(3): p. 031001.

191. Hassan, M., et al., Apoptosis and molecular targeting therapy in cancer. Biomed Res Int, 2014. 2014: p. 150845.

192. Du, Z., X. Tong, and X. Ye, Cyclin D1 promotes cell cycle progression through enhancing NDR1/2 kinase activity independent of cyclin-dependent kinase 4. J Biol Chem, 2013. 288(37): p. 26678-87.

193. Chen, J., et al., Steviol, a natural product inhibits proliferation of the gastrointestinal cancer cells intensively. Oncotarget, 2018. 9(41): p. 2629926308.

194. Gupta, E., et al., Anticancer Potential of Steviol in MCF-7 Human Breast Cancer Cells. Pharmacogn Mag, 2017. 13(51): p. 345-350.

195. Saruc, M., et al., Pancreatic cancer and glucose metabolism. Turk J Gastroenterol, 2009. 20(4): p. 257-60.

196. Pannala, R., et al., Prevalence and clinical profile of pancreatic cancerassociated diabetes mellitus. Gastroenterology, 2008. 134(4): p. 981-7. 


\section{VITA}

Sonam Kumari was born in Bhagalpur, India in 1984. She received her Bachelor of Science in Biotechnology degree from TMBU University, Bhagalpur. Thereafter, she obtained her Master of Science in Applied Microbiology degree from Vellore Institute of Technology (V.I.T) University, India. Further, she came to the United States for pursuing her Ph.D degree. She joined The University of Tennessee Health Science Center, Memphis in Fall 2014 in the Department of Pharmaceutical Sciences. She has presented her research at various conferences. She is a member of AAPS and AACR organizations. She has published articles as first author as well as a co-author. She received her doctorate degree with a major in Pharmaceutics in May 2019. 